\title{
REACTOR PHYSICS PRIMER
}

\author{
By \\ E. H. Lockwood \\ Operational Physics \\ Research and Engineering Operation
}

November 15, 1957

\section{HANFORD ATOMIC PRODUCTS OPERATION RICHLAND, WASHINGTON}

Work performed under Contract No. W-31-109-Eng-52 between the Atomic Energy Commission and General Electric Company

Printed by/for the U.S. Atomic Energy Commission 


\section{DISCLAIMER}

This report was prepared as an account of work sponsored by an agency of the United States Government. Neither the United States Government nor any agency Thereof, nor any of their employees, makes any warranty, express or implied, or assumes any legal liability or responsibility for the accuracy, completeness, or usefulness of any information, apparatus, product, or process disclosed, or represents that its use would not infringe privately owned rights. Reference herein to any specific commercial product, process, or service by trade name, trademark, manufacturer, or otherwise does not necessarily constitute or imply its endorsement, recommendation, or favoring by the United States Government or any agency thereof. The views and opinions of authors expressed herein do not necessarily state or reflect those of the United States Government or any agency thereof. 


\section{DISCLAIMER}

Portions of this document may be illegible in electronic image products. Images are produced from the best available original document. 


\section{TABLE OF CONTENTS}

\section{SECTION A - STRUCTURE OF THE ATOM}

Page

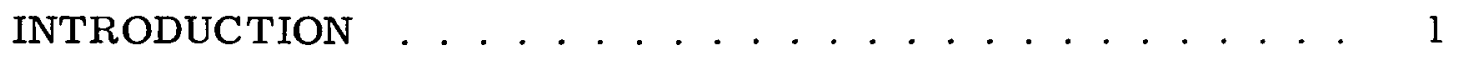

I. BUILDING BLOCKS OF MATTER . . . . . . . . . . 2

Structure of Matter . . . . . . . . . . . . . . . 2

Charge and Mass . . . . . . . . . . . . . . . . 2

Energy and Force .................. . . . . 3

Unit Measurement . . . . . . . . . . . . . . . . . . . . . . . 5

Powers of Numbers . . . . . . . . . . . . . . . . 6

The Proton . . . . . . . . . . . . . . . . 7

The Neutron . . . . . . . . . . . . . . . . . 7

The Electron . . . . . . . . . . . . . . . 8

Atomic and Mass Numbers . . . . . . . . . . . . . . . . . 8

Chemical Properties . . . . . . . . . . . . . . II

II. STRUCTURE OF THE NUCLEUS . . . . . . . . . . . 12

Isotopes . . . . . . . . . . . . . . . . . . . 12

Abundance of Isotopes . . . . . . . . . . . . . . . . . 12

Radioactivity ................... . . . . 13

Radioactive Decay . . . . . . . . . . . . . . . . . . 14

Table I - Uranium-Radium Series . . . . . . . . . . . . 15

Decay Curve . . . . . . . . . . . . . . . . . . . 16

Growth of Activity . . . . . . . . . . . . . 17

III. NUCLEAR PARTICLES . . . . . . . . . . . . . . . . . 19

Beta Particle . . . . . . . . . . . . . . . . . 19

Positron Particle . . . . . . . . . . . . . . . . . . . . . . . . 19

Gamma Radiation . . . . . . . . . . . . . . . . . . . 20

Alpha Particles . . . . . . . . . . . . . . . 20

Mesons . . . . . . . . . . . . . . . . . . . . . 21

Neutrino Particle . . . . . . . . . . . . . . 21

Deuteron . . . . . . . . . . . . . . . 22

Internal Electron Conversion . . . . . . . . . . . . . . 22

K Capture . . . . . . . . . . . . . . . . . . . . . . . 22

Isomeric Transition . . . . . . . . . . . . . . . . 23

IV. NUCLEAR FORCES . . . . . . . . . . . . . 23

Binding Energy . . . . . . . . . . . . . . . 23

Mass Defect . . . . . . . . . . . . . . . . . 26

Equivalence of Mass and Energy . . . . . . . . . . 26

Table II - Units of Mass-Charge-Energy . . . . . . . . . . . 29 
TABLE OF CONTENTS (Contd.)

SECTION A

Page

V. NUCLEAR REACTIONS ............... . . 30

Types of Reactions . . . . . . . . . . . . . . . . 30

Fusion ......................... 31

Artificial Transmutation . . . . . . . . . . . . 33

Equations ................. . . . 34

Mass and Energy Relationship . . . . . . . . . 36

VI. METHODS OF REACTION . . . . . . . . . . . . . 38

Projectiles . . . . . . . . . . . . . . . . 38

Cross Section . . . . . . . . . . . . . . . . . 39

Fast and Slow Neutrons . . . . . . . . . . . . . 40

Neutron Reactions . . . . . . . . . . . . . . . . . . 40

Elastic Impact (Without Capture) . . . . . . . . . . . . . . . 41

Inelastic Impact (Without Capture) . . . . . . . . . . . . 43

Capture of Incident Particle with Emission of a Particle . . . 43

Simple Capture without Emission of a Particle . . . . . . . 44

Resonance Capture . . . . . . . . . . . . 44

VII. NUCLEAR FISSION . . . . . . . . . . . . . . . . . 47

Neutron-Induced Fission . . . . . . . . . . . . 47

Liquid Drop Model . . . . . . . . . . . . . . . . . . . 48

Energy Release . . . . . . . . . . . . . . . 51

Prompt Neutrons . . . . . . . . . . . . . . . . 53

Delayed Neutrons . . . . . . . . . . . . 53

VIII. DETECTION OF NUCLEAR RADIATION . . . . . . . . 54

Ionization . . . . . . . . . . . . . . . . . 54

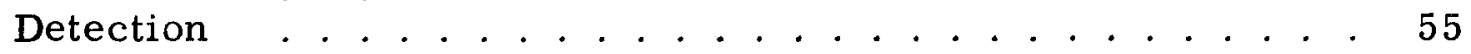

Nature of Radiations . . . . . . . . . . . . . . 57

Alpha Particles . . . . . . . . . . . . . . . . . . . . . . . . 57

Beta Rays . . . . . . . . . . . . . . . . . . 58

Gamma Rays . . . . . . . . . . . . . . . . . 58

Neutrons . . . . . . . . . . . . . . 58 
TABLE OF CONTENTS (Contd.)

SECTION B - PHYSICAL PRINCIPLES OF THE REACTOR PROCESS

Page

I. DESCRIPTION OF THE REACTIONS . . . . . . . . . . . . . 60

Discovery of Plutonium . . . . . . . . . . . . . . 60

Fundamental Nuclear Reactions . . . . . . . . . . . . 61

Formation of Plutonium . . . . . . . . . . . . . . . . 62

Neutron Capture by Uranium-238 . . . . . . . . . . . . . . . . . 62

Fission of Uranium-235 .................. 64

Fission Products .................... . . 65

By-Products of the Reactions . . . . . . . . . . . . 67

Energy of Fission ..................... 68

Dependence of Reactions on Neutron Energies . . . . . . . . . 69

Slowing Down of Neutrons . . . . . . . . . . . . . 71

II. CHAIN REACTION IN THE PILE . . . . . . . . . . . . . . 72

Requirements of a Chain Reaction ............... 72

Chain Reaction ...................... 73

Neutron Loss . . . . . . . . . . . . . . . . . . . . 76

Critical Size ...................... . . . 76

Reflector ......................... . . . . 78

Lattice Spacing ..................... . 79

Multiplication Factor, k . . . . . . . . . . . . . . 82

Excess k . . . . . . . . . . . . . . . . . . . 83

k Infinity . . . . . . . . . . . . . . . . . . 84

k Effective . . . . . . . . . . . . . . . . . . 85

Summary of $\mathrm{k}$ Terms . . . . . . . . . . . . . . . . . . 85

Neutron Balance in a Chain Reaction . . . . . . . . . . . . 86

III. PROPERTIES OF A CHAIN-REACTING PILE . . . . . . . . . 89

General Description . . . . . . . . . . . . . . . 89

Control Rods ....................... . 89

Effect of Operation on Multiplication Factor . . . . . . . . . . . 90

Neutron Distribution ................... 91

Control of Activity .. . . . . . . . . . . . . . . . . . 92

Effect of Delayed Neutrons ................ . 94

Factor of e - Period ................... . 95

Reactivity and Period . . . . . . . . . . . . . . . . . . . 96

Inhour . . . . . . . . . . . . . . . . . . . 97

Semi-Log Paper ...................... . 98

Radiations . . . . . . . . . . . . . . . . . . 98 
TABLE OF CONTENTS (Contd.)

\section{APPENDIXES}

Page

APPENDIX I

Atomic Weights ...................... 101 APPENDIX II

Table of the Elements . . . . . . . . . . . . . . . . 105 APPENDIX III

Chart of the Nuclides ................. 106 


\section{PREFACE}

Training of operating personnel is a vital phase of reactor operation; despite all the limits and safeguards which can be imposed, the ultimate responsibility for reactor safety rests with the individual operating the reactor. Because the operation is constantly being changed and improved, the training program must be flexible, varied, and continuous.

Some effects of power level changes and control rod movements are not evident until several hours after the change. Therefore, it is necessary that the operator anticipate critical control conditions and thoroughly understand all the characteristics of the reactor under various circumstances.

Increased use of electronic equipment, higher power levels, sensitive control, etc., all indicate the urgent need to thoroughly train new personnel, and to upgrade the skill of the present operating group; the degree of skill and know -how of the operating personnel can often mean the difference between safe and unsafe reactor operation.

This manual deals generally with the reactor physics aspects of the processes used at Hanford for the production of plutonium. The text is primarily descriptive, rather than definitive. Process conditions, such as pressures, temperatures, concentrations, activities, etc., stated in this manual are not firm specifications for these processes. Such specifications are covered by "Process Standards", issued separately. This manual is intended primarily for the Reactor Operators, to serve as follows:

1. As an aid toward a better understanding of crucial pile conditions.

2. As a training guide for new personnel.

3. As a source of subject matter for group discussion. 


\section{INTRODUCTION}

The Hanford product is formed from uranium by controlled nuclear reactions. Consequently, a knowledge of the elements of nuclear physics is essential to understanding the process. This manual will introduce the beginner to the fundamentals of this subject.

The field of nuclear physics is so broad and so complex that it would be presumptuous of the author to claim any new or novel ideas in the material presented in the Reactor Physics Primer. Since the material was gathered from many sources, it would be impractical to try to give individual recognition in every case, but the use of such material is hereby acknowledged with thanks. 


\section{SECTION A}

\section{STRUCTURE OF THE ATOM}

\section{BUILDING BLOCKS OF MATTER}

Structure of Matter

All matter is made up of atoms, each of which consists of a positively charged nucleus surrounded by a number of negatively charged electrons, so that the atom as a whole is electrically neutral. All the atoms of matter are made up of suitable combinations of three units. The units are neutrons, protons, and electrons. The neutron and proton are the bricks out of which the nucleus is constructed, around which the electrons move in a miniature solar system, their orbits determined by electrical forces.

It might be well at this point to visualize the nucleus, made up of neutrons and protons, as the "sun" in a solar system, with the electrons as "planets" revolving about the "sun". The electrical attraction between the positively charged protons of the "sun" and the negatively charged electrons or "planets" holds the entire structure together. (See Figure 1).

\section{Charge and Mass}

The weight of the neutron is very nearly equal to that of the proton; in comparison the weight of the electron is almost negligible. The proton and the electron are electrically charged particles, each having identical charges except for sign. The sign of the proton's charge is arbitrarily called positive, the oposite sign of the electron is then called negative. The neutron, as the name implies, is electrically neutral. The mass and charge properties of these particles are summarized as follows:

\begin{tabular}{llc} 
PARTICLE & CHARGE & MASS \\
\cline { 2 - 3 } Proton & Positive & 1 \\
Neutron & Zero & 1 \\
Electron & Negative & $1 / 1840$
\end{tabular}


From the above table it can be seen that the proton carries a single unit of positive charge, equal in magnitude to the electron's negative charge. The mass or weight of the proton is given as one. The neutron is electrically neutral and carries no charge and also has a mass weight of one, though in reality its mass is slightly greater than that of the proton. The electron carries a negative charge, equal in magnitude to the positive charge of the proton, but the mass or weight of the electron is approximately 1840 times less than the mass of either the proton or the neutron.

Energy and Force

The atom itself is so small that no microscope can make it visible to the human eye, not even the electron microscope. It is so minute that over one hundred billion billion are required to form the head of an average small pin. The average atom is approximately $1 / 200,000,000$ th of an inch in diameter measured across the outer-most path of its orbiting electrons. But the nucleus or core of the atom is only some $1 / 10,000$ th of this or $1 / 2,000,000,000,000$ th of an inch. Consequently, the atom is composed largely of empty space, its nucleus forming but a small fraction of the whole. (See Figure 2). Yet this infinitesimally small part of the atom holds energy concentration of fantastic proportions. For instance, a positively charged proton, a particle of the nucleus, repels an immediately adjacent like particle with a force of some forty pounds.

This force is generated in a particle so small and so dense that if a quantity no larger than the size of an average small marble could be assembled of nuclei without intervening voids, it would weigh over 200,000,000 tons or $400,000,000,000$ pounds.

Although statistics balance out the negative and positive charges observed in nature, the preceding discussion shows that tremendous forces are available if we can just tap them. 

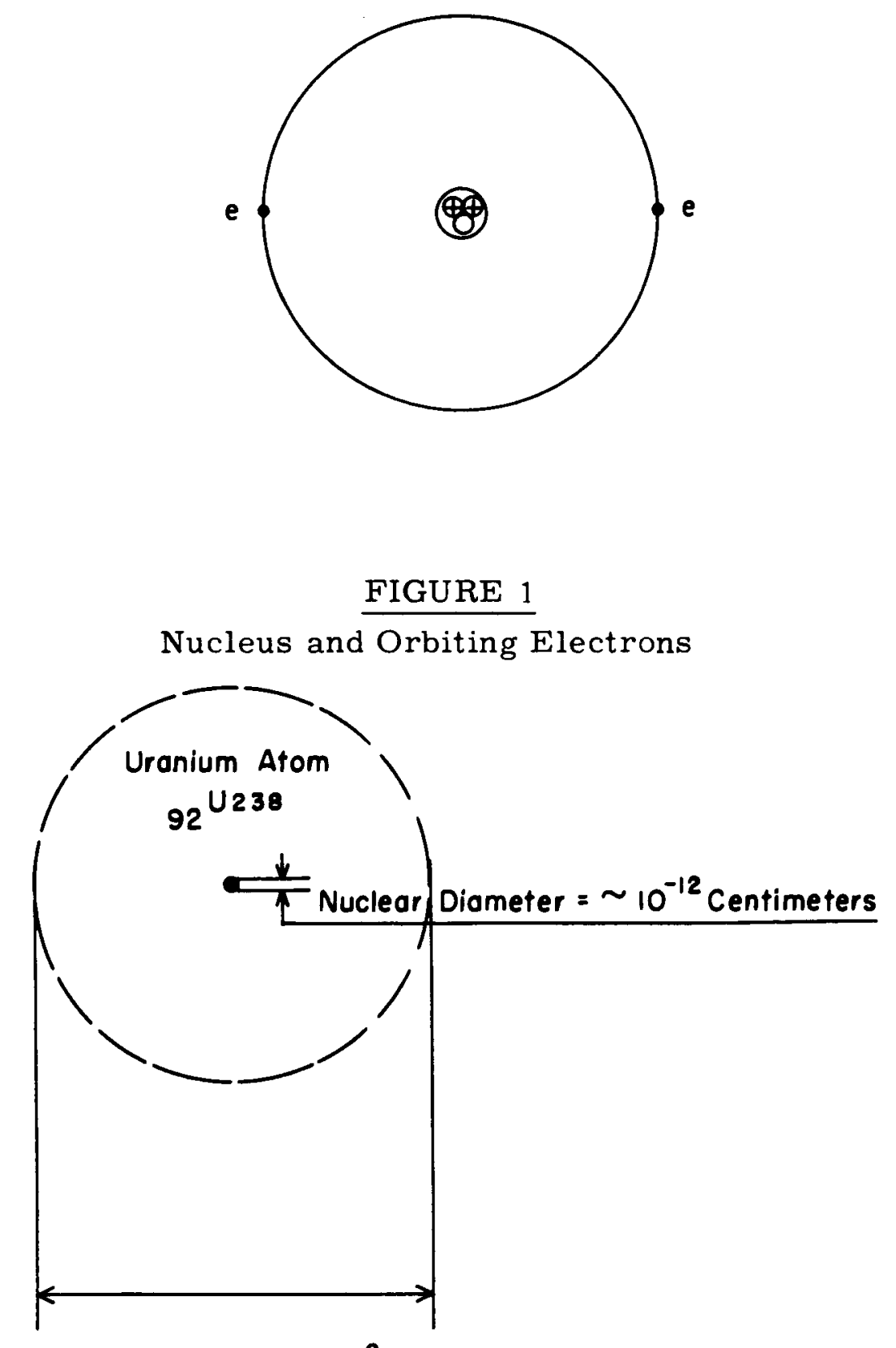

Atomic Diameter $=\sim 10^{-8}$ Centimeters

FIGURE 2 
Unit Measurement

At this point, in order to clarify the order of mass,charge and energy, it is necessary to examine the units established for this purpose. The unit used for the measurement of mass or weight is exactly $1 / 16$ th of the total mass of the common oxygen atom, ${ }_{8} \mathrm{O}^{16}$. This mass or weight is equal to 1. $663 \times 10^{-24}$ grams or about $6 \times 10^{-26}$ ounces or .00000000000000000000000006 ounces. This is known as the atomic mass unit. Measured by this unit, the proton has a mass of approximately $1.00758 \mathrm{amu}$ or atomic mass units.

The unit used to express the electric charge is that possessed by the proton. One "electronic charge" amounts to $1.6 \times 10^{-19}$ coulombs, where a coulomb is the charge conveyed by a current of one ampere flowing for one second.

The unit used to measure energy is the electron volt; this is the kinetic energy acquired by a particle carrying one electronic charge while falling freely through a potential drop of one volt. Since it is so small, relative to nuclear reactions, the unit is usually used in millions and written as Mev (Million electron volts). The comparative size of the electron volt and the more familiar units of energy are presented in the following table:

$$
\begin{aligned}
1 \mathrm{ev} & =1.6 \times 10^{-12} \mathrm{erg} \\
1 \mathrm{Mev} & =1.6 \times 10^{-6} \mathrm{erg} \\
1 \mathrm{Mev} & =1.18 \times 10^{-13} \mathrm{foot}-\text { pounds } \\
1 \mathrm{Mev} & =3.83 \times 10^{-17} \mathrm{~kg} \text {-cal } \\
1 \mathrm{Mev} & =4.45 \times 10^{-20} \text { kilowatt-hours }
\end{aligned}
$$

Energy of thermal agitation of molecules at room temperature is approximately $1 / 40 \mathrm{ev}$.

Energy of combustion (burning one atom of carbon with two atoms of oxygen is approximately five ev).

Energy of alpha particles from naturally radioactive elements is about five Mev。 
Energy of fastest alpha particle accelerated by cyclotron is about 25 Mev.

Energy released in the fission of uranium nucleus is about $200 \mathrm{Mev}$.

Energy released by latest super-accelerator designs, such as the Brookhaven Cosmotron, is of the order of a few Bev (billion electron volts).

\section{Powers of Numbers}

In the table above and throughout the manual the powers of numbers are used as a short way of indicating the size of any given number. For example, $2 \times 2 \times 2 \times 2=16$ can be written simply as $2^{4}=16$. The small number (four) is called the exponent or power of the base number. Similarly, $16 \times 16=256$ can be written as $16^{2}=256$. Thus $10^{1}=10,10^{2}=100,10^{3}=1,000$. In the case where the exponent has a minus sign before it, the inverse of multiplication is indicated. Some examples of the powers of 10 (for plus and minus values) are shown below:

$$
\begin{array}{ll}
10^{0}=1.0 & 10^{0}=1.0 \\
10^{-1}=.1 & 10^{1}=10 \\
10^{-2}=.01 & 10^{2}=100 \\
10^{-3}=.001 & 10^{3}=1000 \\
10^{-4}=.0001 & 10^{4}=10,000 \\
10^{-5}=.00001 & 10^{5}=100,000 \\
10^{-6}=.000001 & 10^{6}=1,000,000 \\
10^{-7}=.0000001 & 10^{7}=10,000,000 \\
10^{-8}=.00000001 & 10^{8}=100,000,000
\end{array}
$$

For convenience of writing and manipulation, numbers are often expressed as factors of appropriate powers of 10. The following example will illustrate: 


\begin{tabular}{|c|c|c|}
\hline $3,450,000$ & may be written & $3.45 \times 10^{9}$ \\
\hline 345 & may be written & $3.45 \times 10^{2}$ \\
\hline 34 & may be wri & $3.45 \times 10^{-1}$ \\
\hline 000345 & may & $3.45 \times 10^{-7}$ \\
\hline
\end{tabular}

The Proton

The nucleus of the atom is made up of two important parts and several other particles that will be discussed later. Chief of the more important parts is the proton. This particle has a mass or weight of 1.00758 mass units, carries a positive charge of electricity, and exerts the most influence in the atomic scheme of things. The number of protons in the nucleus is the determining factor in the element the atom forms. For example, all atoms having six protons in their nucleus are carbon, those having seven protons are nitrogen, and those with 92 protons are uranium. In short, the number of protons determines the element, and the moment that the number of protons can be determined, the element of which the atom is a part can be named. Likewise, it is the number of protons that determines the number of electrons revolving about in the atom. All atoms are electrically neutral; that is, their electrical charges are balanced. The electron has a negative charge exactly equal to the positive charge of the proton. In chemical reactions there is a rearrangement of the outer electrons, but the nucleus remains the same. As the proton count controls the number of electrons, it also establishes the chemical properties of an element, since all substances having the same number of protons in their nucleus will have the same chemical properties. The Neutron

Next in importance of the nuclear particles is the neutron. The neutron, as the name implies, is electrically neutral. It has a mass, or as used non-technically a weight of 1.00893 amu (atomic mass units) slightly more than that of the proton. Its diameter is approximately the same as that of the proton, and it of course carries no charge. Neutrons exist in the nuclei, their number in a given nucleus having a direct effect on the mass of that atom and even on its radioactive stability, but not on its chemical properties. 
The Electron

The third major unit of the atom is the electron. This particle has a mass of 0.000548 mass units, a mere $1 / 1840$ of the mass of either the proton or the neutron. Small in mass as compared to the other units of the atom, the electron exerts a tremendous force in the universe. The electrons do not all lie in the same orbit or shell; the arrangement of the electrons around the nucleus is always in a set pattern or order. They are arrayed in several layers known as shells. In the inner shell next to the nucleus, the maximum number of electrons is two, as in the helium atom. The second layer holds as many as eight, the third layer or shell can contain from 9 to 18, the fourth shell can contain up to 32 electrons, the fifth layer drops to 18 , the sixth to 8 , the seventh and last layer again drops to a total of 2 electrons. (See Uranium, Figure 3).

Most of the seemingly solid atom is just empty space. In fact if the force of the electrons could be overcome and all matter could be crushed together until the nuclei were in contact, instead of being separated by the distance of these electron sheels, matter would weight about two billion tons per cubic inch and the earth would be only about one-fifth of a mile in diameter.

\section{A tomic and Mass Numbers}

Since the charge of the proton is identical with that of the electron, the number of protons in an atom of an element is the same as the number of electrons. This number (the number of protons) is called the "atomic number" of the atom. (Note the vertical numbering in the chart of the nuclides in the appendix).

The total number of particles in the nucleus, the sum of the neutrons and protons, is called the "mass number" of the atom. (See the horizontal numbering in the nuclide chart). The mass number is very nearly equal to the "atomic weight" of the atom for the low atomic numbers, but becomes relatively larger for the high atomic numbers; "binding" forces holding the particles together in these heavier atoms account for this discrepancy in weights (see Appendix I).

UNCLASSIF1ED 


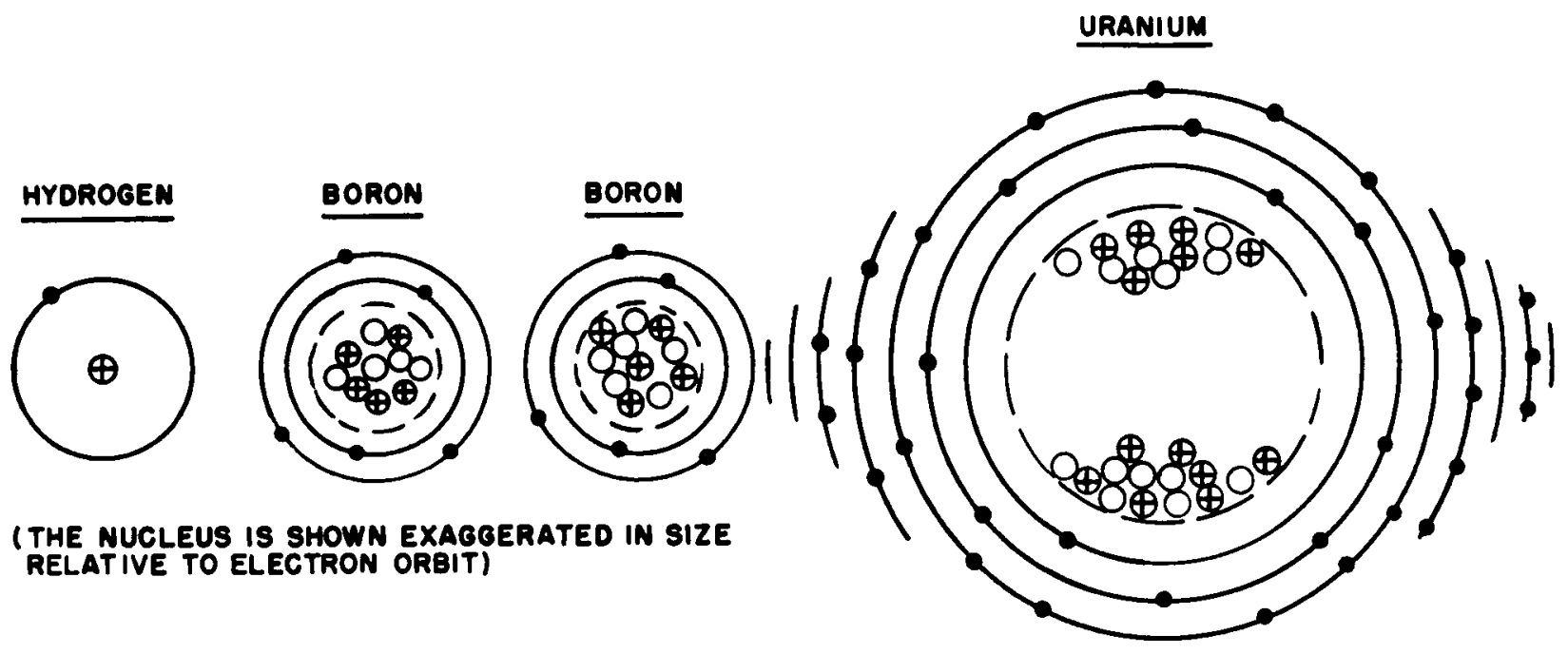

7 ORBITS CONTAINING A TOTAL OF 92 ELECTRONS

FIGURE 3

Structure of Elements

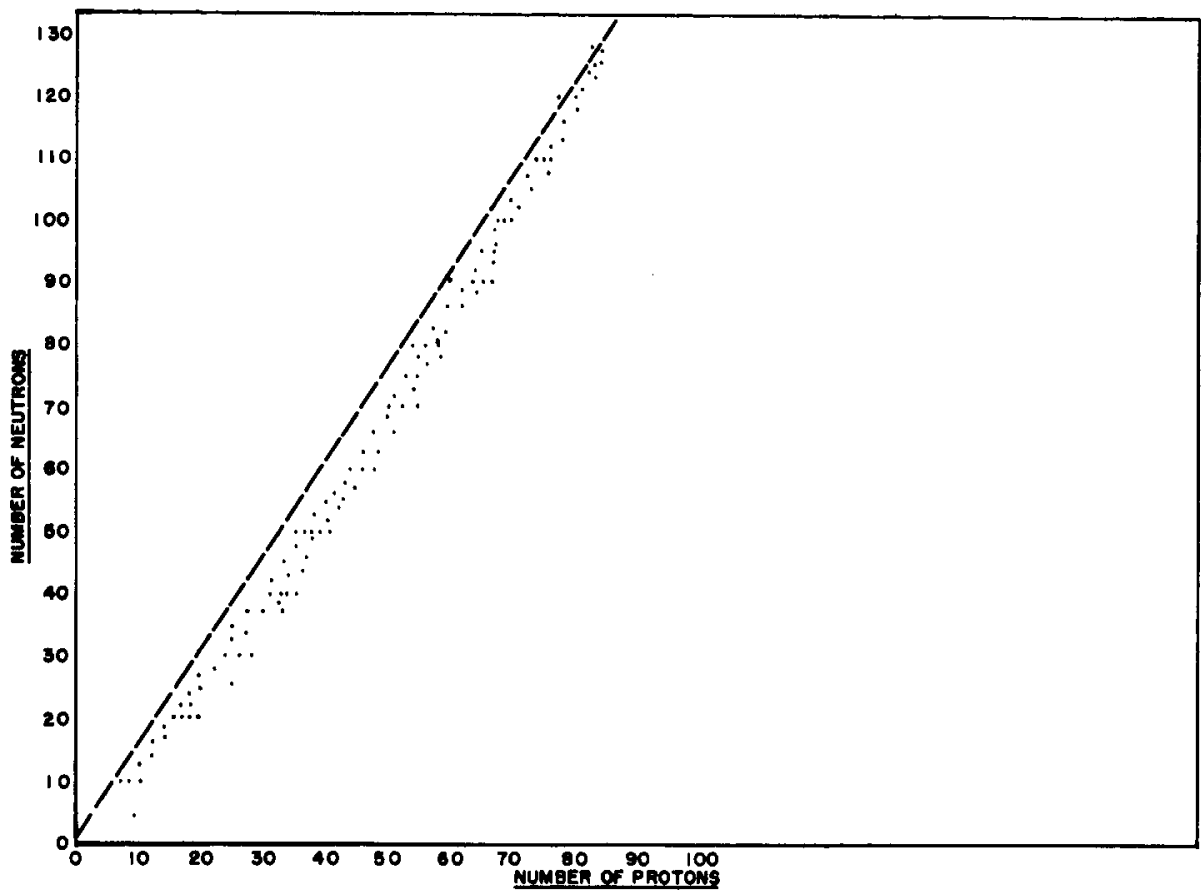

FIGURE 4

Number of Neutrons and Protons in Stable Nuclei 
In nuclear physics, the nucleus is expressed by a symbol which is the chemical abbreviation for the element with the atomic number as a subscript (below) and the mass number as a superscript (above). The arrangement of the symbol with examples, is as follows:

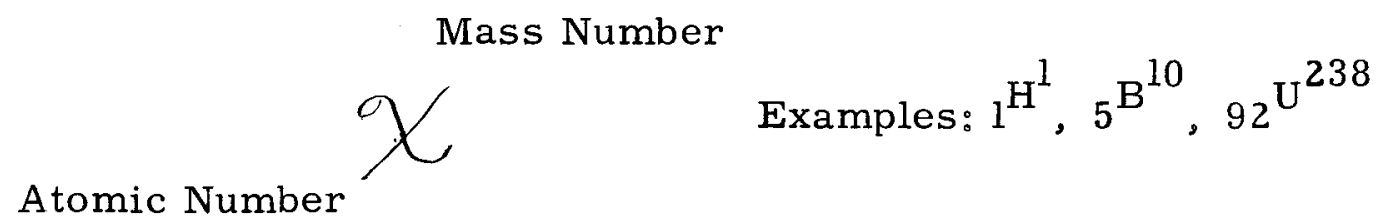

From the examples above:

$\mathrm{H}, \mathrm{B}, \mathrm{U}=$ Abbreviation for Hydrogen, Boron, and Uranium

Atomic Number (below) = number of protons in nucleus

Mass Number (above) $=$ total number of protons and neutrons in nucleus.

In the first example $\mathrm{I}^{\mathrm{H}}$, the $\mathrm{H}$ is the abbreviation for hydrogen, the subscript 1 is the atomic number indicating the presence of 1 proton. The superscript 1 is the mass number and indicates that this hydrogen nucleus consists of one proton only. Hydrogen with simply a proton is called ordinary hydrogen, whereas the hydrogen isotope containing one proton and one neutron is called deuterium and is designated as $1^{H^{2}}$. In the next example $5^{\mathrm{B}^{10}}$, the $\mathrm{B}$ is the abbreviation for boron, the subscript 5 is the atomic number indicating 5 protons, the super-script 10 is the mass number, indicating the presence of 5 neutrons in addition to the 5 protons (the total number of particles in the nucleus is the mass number). In the third example $92 \mathrm{U}^{238}$, the $\mathrm{U}$ is the abbreviation for uranium, the subscript 92 is the atomic number indicating 92 protons present, the superscript 238 is the mass number (the total of particles present in the uranium nucleus). Therefore, the mass number minus the atomic number equals the number of neutrons present. In this case $238-92=146$ neutrons. 
In the table below note how the symbols are formed from the structure of the elements:

Element:

Number of Protons

Number of Neutrons

Mass Number

Atomic Number

Symbol

\begin{tabular}{ccccc} 
HYDROGEN & BORON & & BORON & URANIUM \\
\cline { 5 - 6 } 1 & 5 & 5 & 92 \\
0 & 5 & 6 & 146 \\
1 & 10 & 11 & 238 \\
1 & 5 & 5 & 92 \\
${ }_{1} \mathrm{H}^{1}$ & $5^{\mathrm{B}^{10}}$ & $5^{\mathrm{B}^{11}}$ & ${ }^{11} \mathrm{U}^{238}$
\end{tabular}

Schematic drawings of the structure of the four elements in the above table are presented in Figure 3.

\section{Chemical Properties}

It is the atomic number, not the mass weight, that determines the chemical nature of the element. This is because the chemical properties depend on the electrons which are shared between atoms to form chemical compounds. Since the number or protons is equal to the number of electrons in an electrically neutral atom, we can say that the number of protons in the nucleus determines the chemical properties of the element. For a given element the atomic number, apart from a few exceptions, is equal to the number of the element in order of increasing weight. Thus, the atomic number of hydrogen is 1 , helium 2, lithium 3, beryllium 4, boron 5 , and so on up to 92 for uranium. All other elements existing in nature lie in between atomic number 1 for hydrogen and atomic number 92 for uranium. A number of heavier elements such as plutonium, atomic number 94, have been made artificially; these are indicated in the charts on atomic weight and of the nuclides in the Appendix. A periodic chart of the elements, showing their related chemical families, is also shown in the Appendix. 


\section{STRUCTURE OF THE NUCLEUS}

\section{Isotopes}

The number of electrons in a neutral atom determines the chemical properties of the element. Because the number of protons is equal to the number of electrons in an electrically neutral atom, we can say that the number of protons in the nucleus determines the chemical properties of the element. Therefore, it is the atomic number (number of protons) and not the mass number (total number of protons and neutrons), that determines the chemical nature of the element.

On the other hand it is possible for nuclei of an element, all of which contain the same number of protons, to have different numbers of associated neutrons. Atoms with nuclei containing the same number of protons (same atomic number), but with different numbers of neutrons (different mass numbers) are called "isotopes". To illustrate the difference in the structure of isotopes, two isotopes of boron are shown in Figure 3. The isotope on the left has five neutrons, the isotope on the right has six neutrons. (Also note the isotopes on any horizontal row in the chart of the nuclides in the appendix.)

\section{Abundance of Isotopes}

The majority of the elements found in nature are present in more than one form, although their chemical properties are identical. Typical of these is hydrogen which is present in two stable isotopic forms, one having a mass number of $1,\left({ }_{1} \mathrm{H}^{1}\right)$ and the other a mass number of $2\left(1^{\mathrm{H}^{2}}\right)$. Oxygen is present in three stable isotopic forms with mass numbers of 16, 17, and 18. Tin has more than ten such stable forms.

Uranium exists in nature in three isotopic forms with mass numbers of 234, 235, and 238. In all some 280 staple isotopes occur in nature, but we can produce over 800 unstable or radioactive isotopes by artificial means. Thus several kinds of each element are possible, the various kinds being called "isotopes" of that element. The number of neutrons associated with the protons of a given element determines which isotope is obtained. 
$\mathrm{U}^{238}$ is the heaviest element found in nature but it is never found in a pure state. It is always in a mixture of the isotopes $\mathrm{U}^{238}, \mathrm{U}^{235}$, and $\mathrm{U}^{234}$. About 99. 28 per cent of the mixture is $\mathrm{U}^{238} ; \mathrm{U}^{235}$ accounts for 0.712 per cent, approximately one part in 140 , whereas the rare $\mathrm{U}^{234}$ forms but 0.006 per cent of the total mass. All are radioactive isotopes whose very long half-lives listed below, account for their being found in nature.

ISOTOPE HALF-LIFE

$\mathrm{U}^{238}$

$\mathrm{U}^{235}$

$\mathrm{U}^{234}$
$4.51 \times 10^{9}$ years $(4,510,000,000$ yrs.)

$7.07 \times 10^{8}$ years ( 707, 000,000 yrs.)

2. $35 \times 10^{5}$ years (
\% ABUNDANCE

99.28

0.712

0.006

\section{$\underline{\text { Radioactivity }}$}

All of the elements can be made to take a radioactive isotopic form by artificial means. None of these radioactive forms is stable and in time another element will be produced. In many cases the change continues and a whole new series of elements is formed, each changing into another until the element finally formed is no longer a radioactive isotope and is thus stable.

In stable nuclei the ratio of the number of neutrons to the number of protons falls in a fairly well defined but rather narrow range of values (See Figure 4, page 9). If for some reason the ratiofalls outside of this range, the nucleus is unstable. The unstable nucleus is said to be "radioactive". Whether or not an isotope is radioactive depends upon the ratio of neutrons to protons in the nucleus.

The moment that there are more neutrons present than required within the stability ratio, the isotope will become radioactive. Also, if the number of protons is less than the required ratio for stability, the isotope will be radioactive. Thus, the "stable" nucleus is not radioactive, the "unstable" nucleus is radioactive. The unstable nucleus, being radioactive, will tend to adjust its neutron-proton ratio with energy emission 
to acquire stability by a process called "radioactive decay". In radioactive decay the unstable nucleus adjusts its neutron to proton ratio by changing a neutron to a proton or a proton to a neutron, depending on which change results in a more satisfactory neutron-proton ratio. The time consumed in the adjustment process may vary for the average atom from a fraction of a second to millions of years.

\section{$\underline{\text { Radioactive Decay }}$}

The emission of radiation and subsequent change to another element is known as "radioactive decay". Each radioactive isotope has a characteristic rate of decay and to date no means has been found that will alter this rate of decay, regardless of the amount of the material present. The rate of decay is measured in terms of the period of time required for the radiation to lose one half of its initial activity; the isotopes are then said to have a "half-life" of a certain length of time. The length of the half-life is not a function of the mass of the material. There is an amazing difference in the lengths of the half-lives. For instance, polonium-212 has a half-life of but $3 \times 10^{-7}$ seconds $\left(.0000003 \mathrm{sec}_{\text {。 }}\right)$ whereas thorium-232 has a half-life of $1.39 \times 10^{10}$ years $(13,900,000,000$ years $)$.

On earth we find a total of 42 radioactive isotopes and all are of the heavier elements near the end of the periodic table; the reason for their existence is that they all possess very long half-lives. In these isotopes we find an excellent example of how a whole series of elements may be formed by the radioactive decay of an isotope. For instance radium, first of the radioactive isotopes to be exploited, is a product of the radioactive decay of uranium-238. Uranium-238, uranium-235, and thorium-232, all radioactive isotopes, are the initial source of thirty-nine of the forty-two radioactive isotopes found on earth. In time all these materials will become a "stable" isotope of lead.

The uranium-radium series of natural radioactive decay is shown in Table I. Notice that the loss of an alpha particle $(\alpha)$ lowers the charge 
TABLE I

URANIUM-RADIUM SERIES

\begin{tabular}{|c|c|c|c|c|}
\hline Element & $\begin{array}{c}\text { Mass } \\
\text { Number }\end{array}$ & $\begin{array}{l}\text { Atomic } \\
\text { Number }\end{array}$ & $\begin{array}{c}\text { Radia- } \\
\text { tion }\end{array}$ & Half - Life \\
\hline Uranium (Uranium I) & 238 & 92 & $\alpha$ & $4.51 \times 10^{9} \mathrm{yrs}$ \\
\hline Thorium (Uranium $\mathrm{X}_{1}$ ) & 234 & 90 & $\beta^{-}$ & 24.1 days \\
\hline Protactinium (Uranium $\mathrm{x}_{2}$ & 2) 234 & 91 & $\beta^{-}$ & 1. $14 \mathrm{~min}$. \\
\hline Protactinium (Uranium $Z$ ) & ) 234 & 91 & $\beta^{-}$ & 6. $7 \mathrm{hrs}$. \\
\hline Uranium (Uranium II) & 234 & 92 & $\alpha$ & $2.3 \times 10^{5} \mathrm{yrs}$. \\
\hline Thorium (Ionium) & 230 & 90 & $\alpha$ & $8.0 \times 10^{4}$ \\
\hline Radium & 226 & 88 & $\alpha$ & 1622 yrs. \\
\hline Radon & 222 & 86 & $\alpha$ & 3. 82 days \\
\hline Polonium (Radium A) & 218 & 84 & $\alpha$ & $3.05 \mathrm{~min}$. \\
\hline Lead (Radium B) & 214 & 82 & $\beta^{-}$ & $26.8 \mathrm{~min}$. \\
\hline Bismuth (Radium C) & 214 & 83 & $\alpha, \beta^{-}$ & $19.7 \mathrm{~min}$. \\
\hline Polonium (Radium C') & 214 & 84 & $\alpha$ & $1.5 \times 10^{-4} \mathrm{sec}$ \\
\hline Thallium (Radium $\mathrm{C}^{\prime \prime}$ ) & 210 & 81 & $\beta^{-}$ & 1. $32 \mathrm{~min}$. \\
\hline Lead (Radium D) & 210 & 82 & $\beta^{-}$ & 22.2 yrs. \\
\hline Bismuth (Radium E) & 210 & 83 & $\beta^{-}$ & 5.0 days \\
\hline Polonium (Radium F) & 210 & 84 & $\alpha$ & 138. 3 days \\
\hline Lead (Radium G) & 206 & 82 & Stable & \\
\hline
\end{tabular}


(atomic number) by two and the mass (mass number) by four; loss of a negative beta particle $\left(\beta^{-}\right)$raises the charge (atomic number) by one without materially affecting the mass (mass number). In most cases an atom breaks up every time in the same way, yielding a second atom plus an alpha $(\alpha)$ or a beta $(\beta)$ particle plus some small excess amount of energy in the form of gamma $(\gamma)$ radiation.

Decay Curve

Because the radioactive decay is reduced by one-half during the "halflife" of an isotope, its age can be determined by measuring the amount of radioactivity it possesses. In this manner it has been possible to accurately fix the age of certain elements and even fossils and so form a more accurate estimate as to the absolute age of Earth. Radioactive isotopes, even if present in less than one-billionth of a gram in weight, can be readily detected by radiation measuring instruments such as the Geiger counter. The halflife can be computed from a plot of the activity versus decay time; the resultant curve is called a "decay curve". The decay curve of artificially produced indium $\left(49^{\operatorname{In}}{ }^{116}\right)$ which has a half-life of 54 minutes is shown in Figure 5. The rate of decay, or activity, of a radioactive substance can be expressed by the relation:

$$
\begin{aligned}
\text { Activity } & =\text { initial activity } x \mathrm{e}^{-\frac{0.69 t}{\tau}} \\
\text { where } \mathrm{e} & =\text { base of natural logarithms }=2.718 \\
\mathrm{t} & =\text { decay time } \\
\tau & =\text { half-life of the given isotope }
\end{aligned}
$$

The activity of a radioactive material is expressed in terms of the number of disintegrations of its atoms which occur each second. When the disintegrations per second are equal to $3.7 \times 10^{10}$, the sample is said to have one "curie" of activity. 
Growth of Activity

When a sample is being activated by irradiation, the activity increases in the same manner of approaching saturation as current increases in a circuit which contains an inductance or as the voltage increases across a condenser upon application of voltage. A growth curve is illustrated in Figure 6. This figure also shows the decay of activity after the irradiation is stopped. The level to which the activity rises with extended irradiation depends on the cross section and the half-life of the element, assuming that equal numbers of atoms are present. The growth curve can be represented by the relation:

$$
\begin{aligned}
\text { Activity } & =\text { saturated activity } x\left(1-e^{-\frac{0.69 t}{\tau}}\right) \\
\text { where } e & =\text { base of natural logarithms }=2.718 \\
t & =\text { irradiation time } \\
\tau & =\text { half-life of the given isotope }
\end{aligned}
$$

The saturated activity is the activity which the material would acquire after infinite time of irradiation. This activity is proportional to the cross section of the nuclei irradiated and the number of projectiles which strike the target material per unit time, as well as the decay rate of the isotope being formed. Practically saturated activity can be reached in the finite time equal to four half-lives; irradiation beyond this time does not sensibly increase activity.

In most cases more than one element is present in the irradiated substance, and irradiation results in a complex growth and decay curve. In many instances, the radioactive nucleus does not become stable with the emission of a single electron. This situation gives rise to the so-called "radioactive series" (Table I) in which a succession of radioactive nuclei is formed, each with its characteristic half-life. 


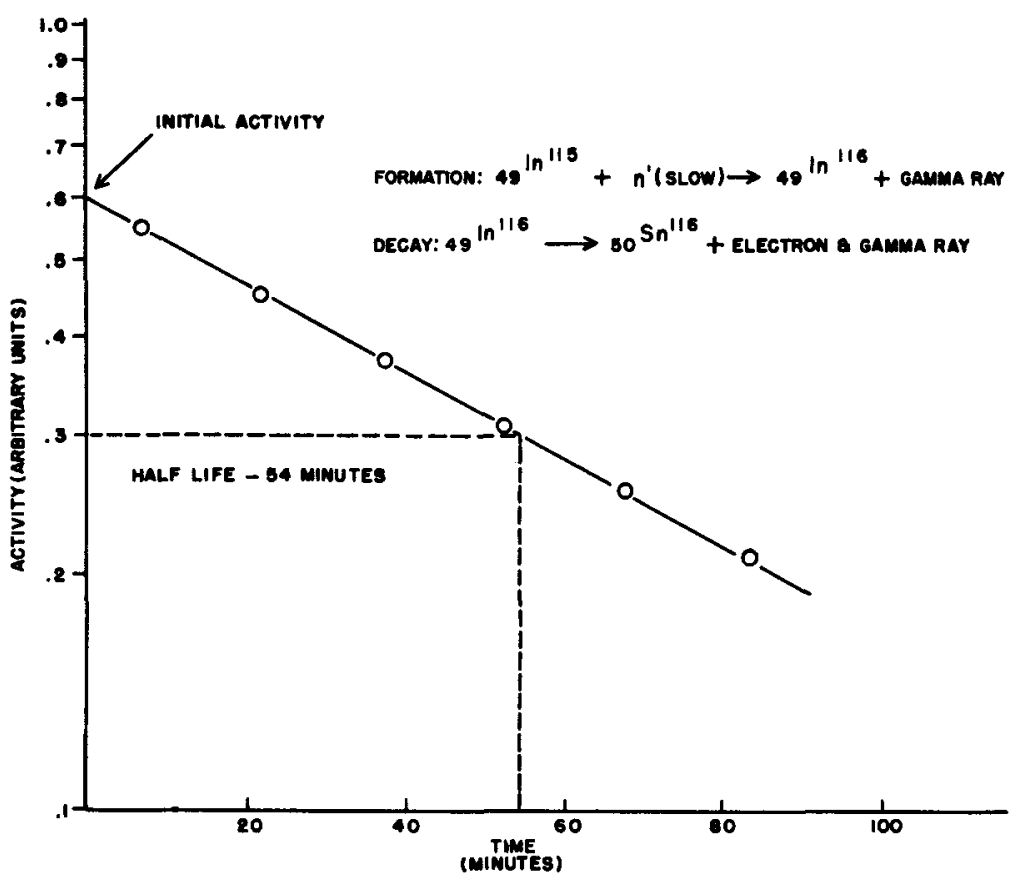

FIGURE 5

Decay Curve of Indium $\left(49^{\operatorname{In}^{116}}\right)$

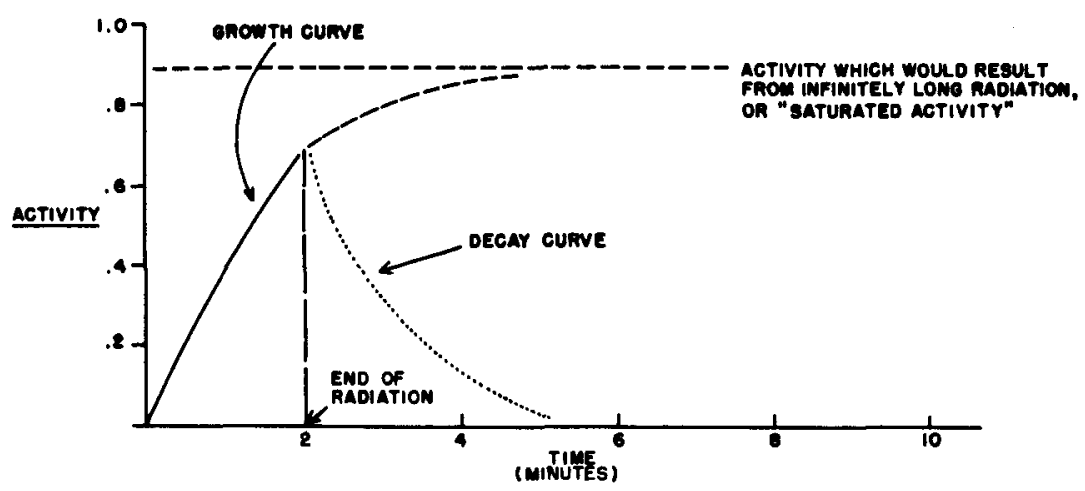

FIGURE 6

Growth and Decay Curves 
HW -51856

\section{NUCLEAR PARTICLES}

Physicists now recognize a number of different nuclear particles. As stated before, the proton and neutron are the particles from which the nuclei of all atoms are built. For this reason they are often called nucleons (part or particles of the nucleus). The alpha particle and the deuteron are groupings of protons and neutrons, and behave as individual particles. These and other particles about to be discussed are known or are believed to result from various changes the nucleus can undergo.

Beta Particle

When there are too many neutrons present for stability, the neutron will change and become a proton. A neutron is electrically neutral; this results from the fact that it contains equal balance between positive and negative charge. A neutron converts itself into a proton by ejecting a negative charge in the form of an electron, called a "beta particle". Because of the extremely small mass of the electron (0.000548), the mass does not change significantly. As the number of protons has changed in the nucleus, the atomic number (number of protons) has likewise changed and the product is another element.

This second element will be less radioactive than the first. If it is still radioactive, another neutron will change into a proton and another electron will be ejected (number of protons and electrons must be equal) as a beta particle. In turn, the changed number of protons in the nucleus will result in the product being a still different element. Similar changes will continue to occur until the neutron-proton ratio reaches the stability range of the element.

Positron Particle

The positron, or positive beta particle, differs from the electron only in the sign of its charge and its length of life. Like negative beta particles, or electrons, the positron has a mass of $1 / 1840$ the mass of a proton and is released from the nucleus at the instant it is formed. 
When there are too many protons present for stability, the proton will change and become a neutron. A proton converts itself into a neutron by ejecting the positive charge as a particle having the same mass as the electron but of opposite charge. This particle is called a "positron". As the number of protons is again changed the atomic number has likewise changed and the product is a different element from the original.

Here again the radiation of a very light particle, a positron in this case, is called beta activity with the qualifying term of positive. If the product is still radioactive, similar changes of a proton to a neutron and the emission of a positron will continue to occur until the neutron-proton ratio reaches the stability range of the element. The vast majority of known radioactive atoms are electron instead of positron emitters. Ordinarily, the life span of a positron is only a small fraction of a second for it combines with a free electron and the two disappear in a burst of gamma rays.

Gamma Radiation

Gamma rays are unlike all the other particles of nuclear origin because they are electromagnetic radizions - like light and radio waves. As far as their behavior in nuclear physics is concerned, gamma rays exhibit a definite "bundle" characteristic, being emitted as "photons" having considerable momentum. Gamma rays are without rest mass and have no charge. Their loss alters neither the charge nor mass of the nucleus; that is, they come from a shift in the energy level of a given nucleus. They are exactly like high-frequency, or hard, X-rays. They come from many nuclear reactions and are among the radiations emitted by natural radioactive elements. Gamma radiation is very penetrating and is not completely stopped by matter. Instead, its intensity decreases according to an exponential law as it penetrates matter.

Alpha Particles

When protons and neutrons join together in heavy nuclei, they sometimes arrange themselves into certain stable groups. The alpha particle 
is such a group and is built up of two protons and two neutrons. The alpha particle is identical with the nucleus of the helium atom ${ }_{2} \mathrm{H}^{4}$. The alpha particle has a positive charge of two electronic units and a mass of 4. 00278 mass units. The particle is commonly emitted by the heavier elements such as polonium-212, radium-226, uranium-233,235, 238, and plutonium-239 (See Table I, page 16)。 Loss of an alpha particle lowers the mass number by four and the atomic number by two.

\section{Mesons}

Several types of mesons have been identified. The first form of the meson immediately begins a radioactive decay and forms the second type. The first meson which appears when the target material is bombarded with high energy particles is called the "pi meson". It has a mass of some 280 times that of an electron. But in about $1 / 100,000,000$ th of a second, it decays into a neutrino and a "mu meson". The mu meson has a mass of some 200 times that of an electron and exists for about $2 / 1,000,000$ ths of a second. This in turn decays into a beta particle and two neutrinos. Both positive and negative mesons are known, and neutral varieties of both are believed to exist. It is thought that these particles may be the "glue" which sticks the nucleus together.

Neutrino Particle

Another particle in the neutron-proton structure is concerned with beta decay in which the electron is emitted. The difference in energy between the value of the emitted electron and the original neutron (if the neutron is altered to a proton) is not equal to known quantities which are present. To account for the very small difference it is thought that another particle exists in the neutron-proton structure which is termed a "neutrino"。

The neutrino must carry no charge and its mass must be so small that it can not be measured (estimated at two per cent of the mass of an electron). A strange thing about the neutrino is its surprising indifference to ordinary matter. It has been estimated that a neutrino would have to 
travel 300,000 miles in air before it would strike a nucleus. Once formed in a reaction, neutrinos evidently escape from the earth without interference and without detection. An elaborate experiment by Los Alamos Scientists to detect neutrinos at Hanford did not give conclusive results, whereas a more recent and more rigorous attempt by the same group at Savannah River was reportedly successful.

Deuteron

The deuteron is made up of a proton and a neutron, and is identical with the nucleus of deuterium, or heavy hydrogen. A deuteron has a positive charge of one electronic unit and it has a mass of 2. 0146 mass units. The deuteron is important in nuclear physics because it is the most simple of the complex nuclei, and can cause a variety of nuclear reactions. Deuterium or heavy hydrogen is present in natural hydrogen only to the extent of one part in 6400 .

\section{Internal Electron Conversion}

Internal electron conversion occurs in conjunction with gamma ray emission, especially if the energy of the gamma rays is low and the atomic number of the nucleus is high. Instead of being emitted directly, the emitted. gamma ray strikes one of the atom's planetary electrons, knocking it out of the atom. This ejected electron is the one which is internally converted The atom then has a "hole" in one of its orbits and another electron falls in to fill this space. The jumping of this electron from one orbit to the other causes the emission of $\mathrm{X}$-rays.

\section{$\underline{\text { K Capture }}$}

$\mathrm{K}$ capture or electron capture is equivalent to positron emission. But instead of losing its excess positive charge by emitting a positron, the nucleus absorbs a planetary electron to neutralize the charge. Since the $\mathrm{K}$ shell is nearest the nucleus the absorbed electron is usually captured from that shell. Loss of the electron leaves a "hole" in the orbit which is promptly filled by another electron falling into it. As in the case of electron conversion, $\mathrm{X}$-rays are emitted.

\section{UNCLASSIFIED}


Isomeric Transition

Isomers are nuclei with identical numbers of protons and neutrons. However, the nuclei have different energy levels. Isomeric transition is the process involved when a nucleus moves from the higher to the lower energy level. It does this by emitting a gamma ray or by internal electron conversion.

These are the more important nucleons and complete the particles which are generally accepted as being present in the nucleus. The reaction of these particles under nuclear bombardment will be discussed later.

\section{NUCLEAR FORCES}

\section{Binding Energy}

The forces which bind the nucleus together are perhaps the biggest mystery about it. Protons having like charges repel one another with tremendous force. But there are forces of attraction among the nuclear particles which are still greater. The excess of the forces of attraction over those of repulsion holds the nucleus together. The "tightness" of this attracting energy is expressed as the binding energy of the nucleus. The greater the binding energy between protons, the more stable the nucleus is. As shown in Figure 7 , medium weight elements in the neighborhood of iron (mass number 56) have the greatest binding energy per nuclear particle.

There are two opposing types of forces in the nucleus, those of attraction and those of repulsion. The force of electrical repulsion is called the "coulomb" force, and arises because of the like charges of protons. It is a tremendous force, varying inversely as the square of the distance of separation of the charged particles. At nuclear distances two protons undergo an electrostatic repulsion of the order of six tons.

But the forces of attraction in the nucleus called the "nuclear forces" are even greater. They exist between protons $(p-p)$, between neutrons $(n-n)$, and between protons and neutrons $(p-n)$. These forces do not appear to operate until the particles are brought very close together. 
It is believed that a nuclear force exists which has the property of saturation. Just as two hydrogen atoms $(\mathrm{H})$ attract each other very strongly to form a hydrogen molecule $\left(\mathrm{H}_{2}\right)$ and then attract no additional atoms, so two protons and two neutrons tend to unite into an alpha particle with comparable saturation effects. At extremely short distances this force of attraction becomes one of repulsion, which prevents the penetration of one group of its components into its neighbor.

A molecule inside a liquid droplet is attracted equally in all directions by the other molecules around it, and the resultant molecular force on it is zero. But a molecule at the surface of the droplet has no molecules beyond to attract it, and the resultant of all the molecular forces acts towards the center of the droplet. This tendency to pull the surface molecules inward and and shrink the surface as small as possible is called "surface tension". An analogous situation occurs among nuclear particles. The effect of this surface tension type of nuclear force decreases with an increase in mass number (total number of protons and neutrons) of the nucleus just as a large drop changes from a sphere to a flat shape.

For large nuclei the electrical repulsion force tends to overcome the other forces and the binding energy falls. In these heavy nuclei greater stability is attained when a greater proportion of neutrons is present to counteract the electrical forces of the larger number of protons. As shown in Figure 7, the binding energy curve rises rapidly for light nuclei and then begins to level off, as the saturation effects of the alpha-particle groups come into play. Beyond this the curve rises more slowly as a result of decreasing surface tension, reaching a maximum at about $A$, mass number $=56$. Then the curve of binding energy gradually falls as the electrical repulsion forces overcome the effects of surface tension. At the higher values the binding energy is so greatly reduced that the nuclei become unstable and show radioactive disintegration. 


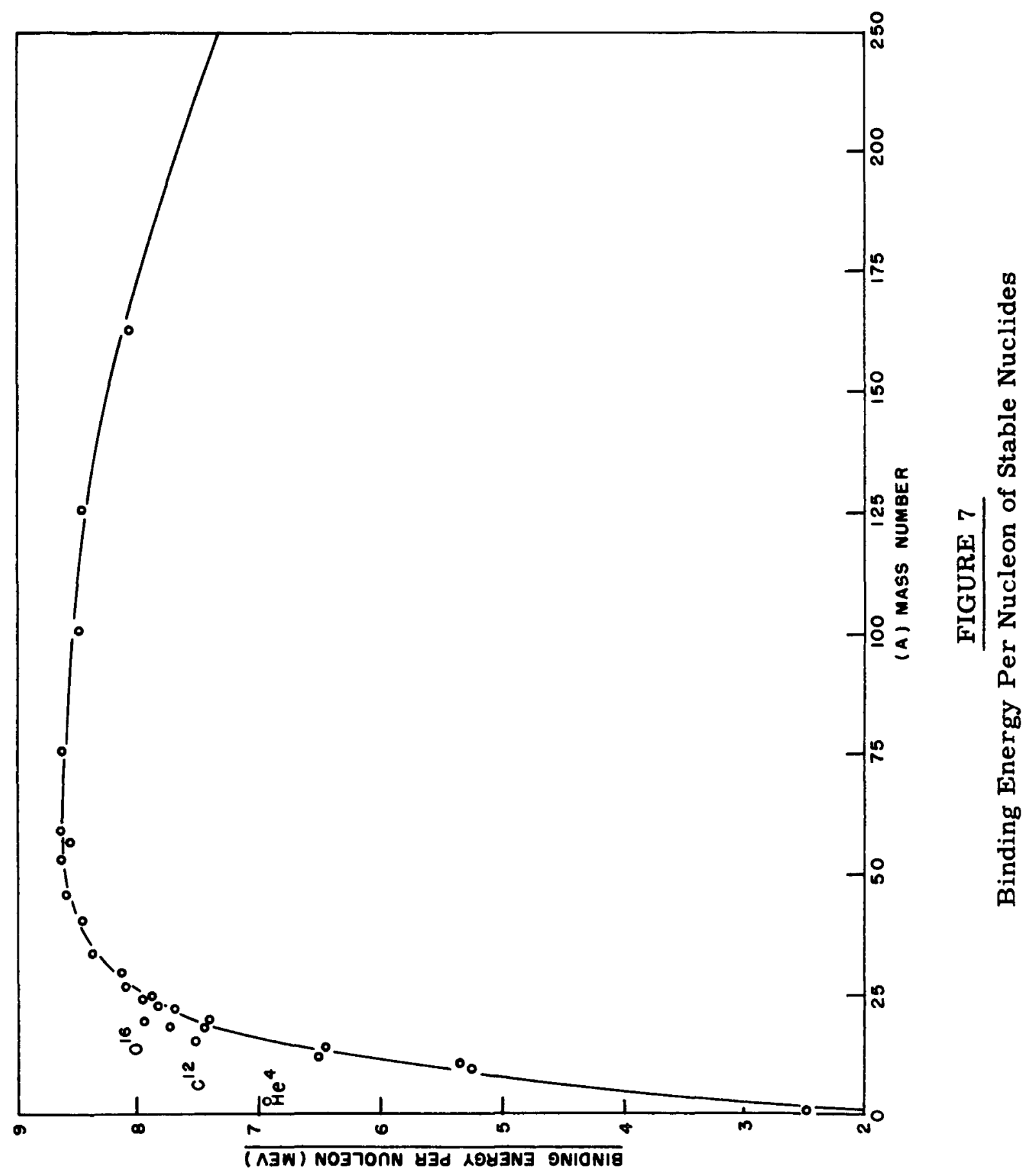


Mass Defect

A nucleus has less mass, and therefore less potential energy, than the individual particles which get together to form the nucleus (See Figure 8). The two protons and two neutrons of a helium nucleus $\left({ }_{2} \mathrm{H}^{4}\right)$ have a mass of 4. 03308 mass units as independent particles. But the actual mass of a helium nucleus is only 4.00278 mass units, 0.03030 mass units less than the mass of the individual particles. This loss is called the "mass defect".

When a nucleus is formed, this small amount of mass disappears and changes into energy which is radiated away. Therefore, the nucleus lacks by this amount the energy necessary to exist as separate particles. This is the binding energy of the nucleus, the energy which sticks the nucleus together or must be added to break it apart. The binding energy is simply the mass defect expressed as an equivalent amount of energy.

Equivalence of Mass and Energy

One of the outcomes of modern physics has been a change in our ideas about matter and energy. We now know that matter and energy are convertible into each other and that the processes go on in even the most commonplace physical and chemical changes. There are isolated nuclear reactions in which matter is completely converted into energy and vice versa. However, so far as we know the particles of matter involved are not massive particles, protons and neutrons, but are positrons and electrons. When a positron is produced, it combines almost immediately with an electron, and the mass of the two particles disappears entirely to form two gamma rays (energy). This process is an example of the complete interconversion of matter and energy. The conclusion that matter and energy are equivalent was first expressed by Albert Einstein in the famous equation:

$$
\begin{aligned}
E & =M C^{2}, \text { or } \\
\text { Energy } & =\text { Mass } x \text { (velocity of light })^{2}
\end{aligned}
$$


This is a general relationship which applies to all matter. It states that energy (E), in ergs, is equal to mass (M), in grams, times the square of the velocity of light, in centimeters per second. An illustration of the equivalence of mass and energy is shown in Figure 9.

A positron and an electron are uniting to form a pair of gamma rays. The mass of the particles (18.22 $\times 10^{-28} \mathrm{gram}$ ) is being transformed into the enrgy (1. $64 \times 10^{-6} \mathrm{erg}$ ) of the rays which release $0.82 \times 10^{-6} \mathrm{erg}$ each. In nuclear physics mass is usually expressed in mass units and energy is expressed in million electron volts (Mev). When so stated, energy (in Mev) equals mass (in mass units) multiplied by 931 . In the case shown the mass of the particles ( 0.00110 mass units) times 931 gives the energy (1.024 Mev) of the pair of gamma rays.

To establish the atomic units of mass charge and energy in more clearly understandable terms they can be expressed as follows: 


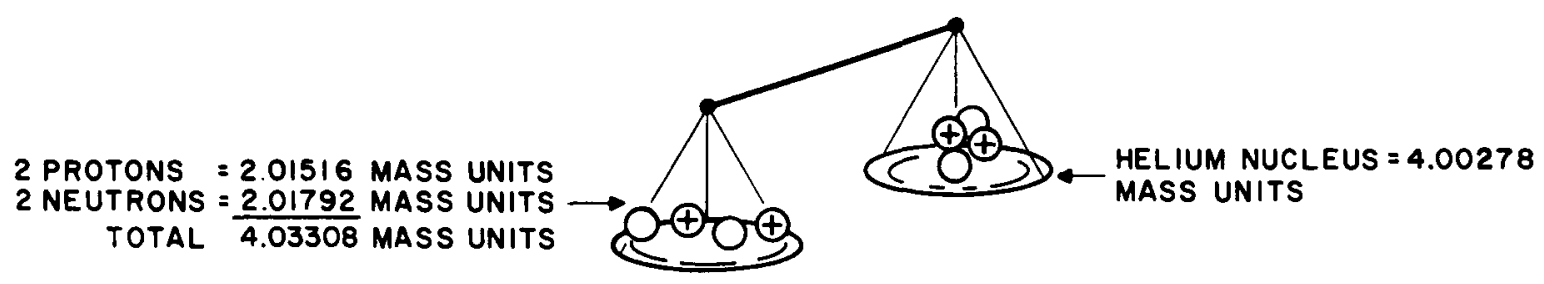
MASS DEFECT $=0.03030$ MASS UNITS

FIGURE 8

Mass Defect

$0.82 \times 10^{-6}$ ERG

$0.512 \mathrm{MEV}$

GAMMA RAY

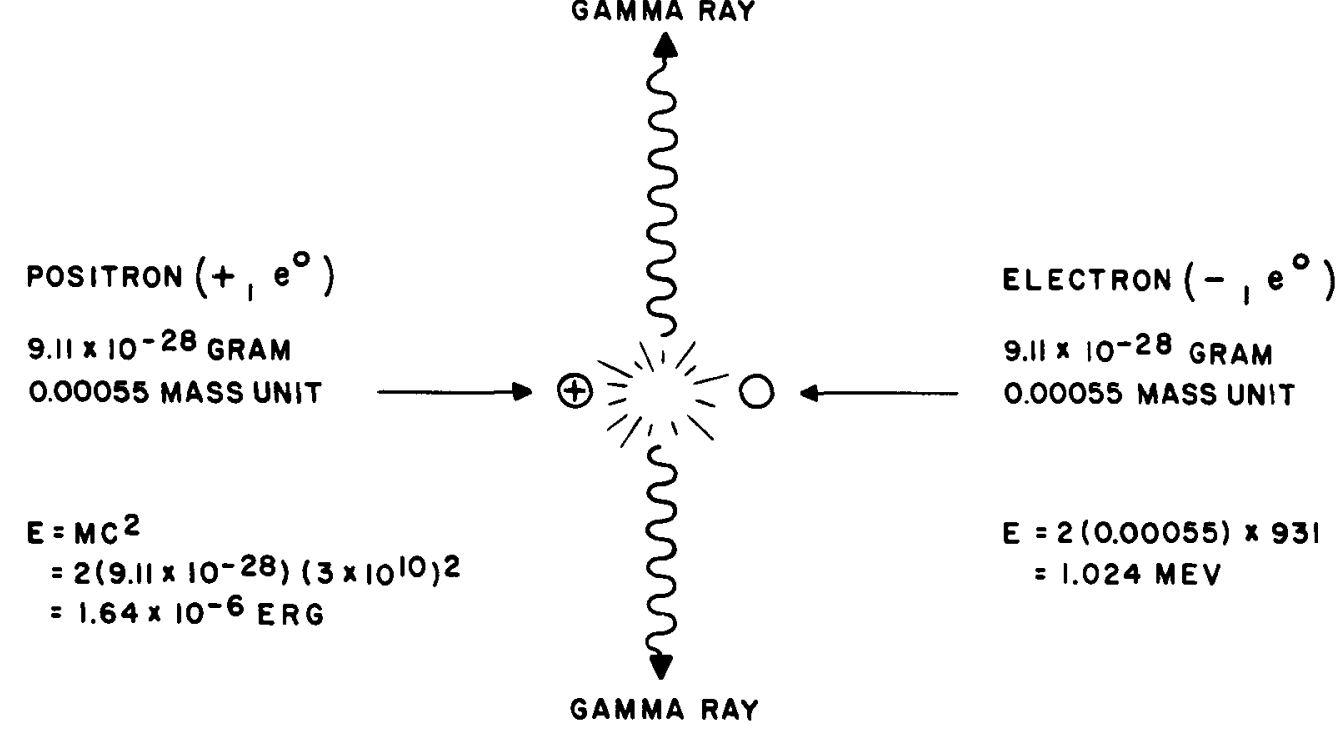

GAMMA RAY

$0.82 \times 10^{-6}$ ERG
$0.512 \mathrm{MEV}$

FIGURE 9

Equivalence of Mass and Energy 
TABLE II

UNITS OF MASS-CHARGE-ENERGY

\begin{tabular}{lll} 
Multiply & \multicolumn{1}{c}{$\frac{\mathrm{By}}{1.07 \times 10^{-3}}$} & \multicolumn{1}{c}{ To Obtain } \\
\hline Mev & $1.60 \times 10^{-6}$ & Atomic Mass Units \\
& $3.83 \times 10^{-14}$ & ergs \\
& $4.45 \times 10^{-20}$ & gram calories \\
Atomic Mass Units & $9.31 \times 10^{2}$ & kilowatt-hours \\
& $1.49 \times 10^{-3}$ & Mev \\
& $3.56 \times 10^{-11}$ & ergs \\
& $4.15 \times 10^{-17}$ & gram calories \\
Ergs & $6.71 \times 10^{2}$ & kilowatt-hours \\
& $6.24 \times 10^{5}$ & Atomic Mass Units \\
& $2.39 \times 10^{-8}$ & Mev \\
& $2.78 \times 10^{-14}$ & gram calories \\
Gram Calories & $2.81 \times 10^{10}$ & kilowatt-hours \\
& $2.62 \times 10^{13}$ & Atomic Mass Units \\
Kilowatt Hours & $4.18 \times 10^{7}$ & Mev \\
& $1.16 \times 10^{-6}$ & ergs \\
& $2.41 \times 10^{16}$ & kilowatt hours \\
& $2.25 \times 10^{19}$ & Atomic Mass Units \\
& $3.60 \times 10^{13}$ & Mev \\
& $8.60 \times 10^{15}$ & ergs \\
& & gram calories \\
& &
\end{tabular}


One erg is the energy required to lift a mosquito about $1 / 2$-inch.

Ten million ergs $(10,000,000)$ is equal to one watt-second of energy.

One watt is the using of one joule of energy every second.

Sixty watts is equal to the power required by a girl weighing 110 pounds, to walk slowly at the rate of one step a second, up a flight of steps, each about six inches high.

A 150 pound man, running upstairs at a rate that carries him up 43 inches per second, is working at the rate of one horse-power.

746 watts is equal to one horse-power in rate of work.

\section{NUCLEAR REACTIONS}

Types of Reactions

Although the main process going on in a reactor is that of atom splitting, there are other types of nuclear reactions going on to form the product in the pile and to cause residual radiation effects. This chapter, therefore, is intended to explain the basic types of nuclear reactions though they do not all occur in a nuclear reactor.

Man has been able to produce hundreds of nuclear reactions artificially. Generally, they are on a small scale and of scientific interest only, and the total energy required to produce the reaction is greater than the energy given back by the reaction. Man-made reactions are caused by hurling atomic particles at the nuclei of atoms. If conditions are right a nucleus is hit, and changes occur in it. Just what happens depends on what nucleus is hit, what particle is used as the "bullet", and how much energy it has.

It is a common misconception that the energy of nuclear reactions comes from the annihilation of appreciable amounts of matter and its complete conversion into energy. However, the energy release from a nuclear fission causes a change in mass of only about $1 / 10$ of one per cent. 
Such a belief is encouraged by speculations which show, for example, that the total energy represented by one pound of matter is equal to that obtained by burning 1-1/2 million tons of coal. In the burning of coal only the outer electrons surrounding the nuclei of the atoms of carbon (coal) are rearranged and the same atoms exist at the end of the reaction as at the beginning. Thus, none of these particles, which make up the mass of all matter, are destroyed in chemical reactions. They are merely rearranged into more stable forms, and the energy released is only that which comes from the difference in the binding energy of the new arrangements. Similar reasoning holds in nuclear reactions, but the energy release per atom is orders of magnitude greater.

More stable nuclei can be formed in two ways - from the combining of light nuclei (fusion) or the splitting of heavy nuclei (fission).

Fusion

Nuclear energy can be released from ordinary matter - a brick, a lump of coal, or a glass of water - simply by bringing it up to a high enough temperature. But this is no easy task, for the easiest of all materials to "ignite", deuterium, requires about one million degrees. The sun (and other stars) easily exceed this temperature. A typical example of the "fusion" process is the formation of helium four nuclei in the interior of the sun.

Under the impetus of the tremendous degree of heat present, an estimated 36,000,000 degrees Fahrenheit, four of the light atoms of hydrogen are fused (fusion) in a thermonuclear reaction into a single atom of helium containing two protons and two neutrons. In this reaction an atom of carbon plays a part, but is eventually returned to its original state. During the course of the reaction, an immense amount of energy is given off and it is this energy which maintains a large part of the energy of the sun.

One proton (hydrogen nucleus) is forced in the carbon nucleus, which throws off gamma rays and becomes nitrogen-13, since it now contains seven protons, and nitrogen is an atom having seven protons. This nitrogen -13 is unstable, and throws off a positron; that is, it gets rid of 
one positive charge, by which a proton becomes a neutron, and becomes carbon-13. Another proton is forced into this, making it nitrogen-14, which again throws out gamma radiation.

We now have a nucleus containing seven protons and seven neutrons. Another proton is thrust into this, making it oxygen-15 (eight positive charges, or protons) with more release of gamma rays. This breaks down into nitrogen-15 by throwing off a positron, in which operation one of the protons becomes a neutron.

Still another proton is forced in. This is too much, it seems, and the atom tosses out a whole bundle this time, the bundle being the stable alpha particle, or helium nucleus ${ }_{2} \mathrm{He}^{4}$. This removes two protons and two neutrons, letting the remaining nucleus revert to its original form of carbon ${ }_{6} \mathrm{C}^{12}$, with six protons and six neutrons, all ready to begin the operation over again.

F our hydrogen nuclei have gone in, and one helium nucleus has been thrown out. Two positrons, or positive charges, have been tossed out which have an extremely short half-life, before breaking down to yeild energy. And there has been a loss of mass in the process, by the release of extremely hard gamma radiation. This gamma radiation is kicked back and forth through all the layers of the sun, working its way outward from the center, being slowly broken down to softer forms of radiation, until, at the outer surface, most of it emerges as light and other radiation.

All that is required to make this operation work is a temperature of about forty million degrees fahrenheit, a pressure of a billion tons (a thousand million tons) per square inch, and a density seven times as great as that of lead.

During a period of one second, about 500 million tons of hydrogen are changed into 496 million tons of helium - and there are then four. million tons of lost mass, which is converted into energy - representing $500,000,000,000,000,000,000,000$ horsepower. About one half of onebillionth of this energy reaches the earth. 
It has been calculated that one pound of mass is equal to six million million $(6,000,000,000,000)$ horsepower of energy. The energy released by the conversion of about two and one-half ounces of mass to energy would be sufficient to supply all the energy requirements of the whole world in all its functions for one second.

If man does learn to control a fusion reaction, he will have unlimited power within his grasp.

Artificial Transmutation

In one of the first artificially induced nuclear reactions, nitrogen nuclei were bombarded with swiftly moving helium nuclei, or alpha particles, from naturally radioactive elements. As a result of the bombardment, the nitrogen nucleus was made to emit a high velocity proton. In such a reaction, the nuclei of the atoms are changed, and completely new and different atoms are formed. Here is a typical reaction in which a helium nucleus hits a nitrogen nucleus, giving a new oxygen nucleus and a proton plus energy. The reaction presented schematically and according to conventional symbols is shown in Figure 10.

A nuclear reaction between colliding nuclei generally becomes more probably the higher the velocity of the bombarding particles. High velocities are required whenever it is necessary to overcome the repellent forces which exist between charged projectiles and target nuclei. Most projectiles consist of nuclei and therefore experience the repellent forces of the similarly charged nuclei. Neutrons, however, are electrically neutral and are neither attracted nor repulsed electrically by the nucleus of an atom. Consequently they can enter the nucleus readily regardless of their energy, that is, regardless of whether or not they are traveling at high speed. In fact, the probability of neutron-induced reactions is generally inversely proportional to neutron velocity.

The helium nuclei used in the above reaction are emitted from their natural source with a speed of about $4 \times 10^{7}$ miles per hour. Even at this 
speed the yield from the reaction is low. Recent advances in high voltage and electronic equipment and techniques have made it possible to accelerate helium nuclei to very much higher speeds with resultant higher yields. The cyclotron, Van de Graaff generator, and linear accelerator are the most popular of the many ingenious devices which have been designed. By their use, other atomic projectiles with high energies are obtained. These projectiles include protons $\left({ }_{1} \mathrm{H}^{\mathrm{l}}\right)$ from ordinary hydrogen and deuterons $\left({ }_{1} \mathrm{H}^{2}\right)$ from heavy hydrogen, in addition to alpha particles from helium.

\section{Equations}

The equation for a nuclear reaction can be written in different ways. No matter how written, the equation is simply a shorthand sentence showing the reacting and product nuclei. In simple reactions a nuclear particle is hurled at a nucleus, which ejects a nuclear particle (that is, a particle from the nucleus) and changes into a different nucleus of nearly equal mass and charge. For example:

$$
{ }_{6} \mathrm{C}^{13}+{ }_{1} \mathrm{H}^{1} \longrightarrow{ }_{7} \mathrm{~N}^{13}+{ }_{0} \mathrm{n}^{\mathrm{l}}
$$

Here a proton $\left({ }_{1} \mathrm{H}^{\mathrm{l}}\right)$ penetrates a carbon nucleus $\left({ }_{6} \mathrm{C}^{13}\right)$ which ejects a neutron $\left({ }_{0} n^{1}\right)$ and changes into a nitrogen nucleus $\left({ }_{7} \mathrm{~N}^{13}\right)$. It should be noted that the sum of the subscripts on the left of the arrow balance the sum of the subscripts (or positive charges) on the right, and the same is true of the superscript (total weight) sums.

In addition to this means of representing a nuclear reaction, there is a more concise scheme. It is as follows:

Bombarded nucleus (incident particle, ejected particle) resulting nucleus.

For the above reaction, this method gives: $C^{13}(p, n) N^{13}$ 


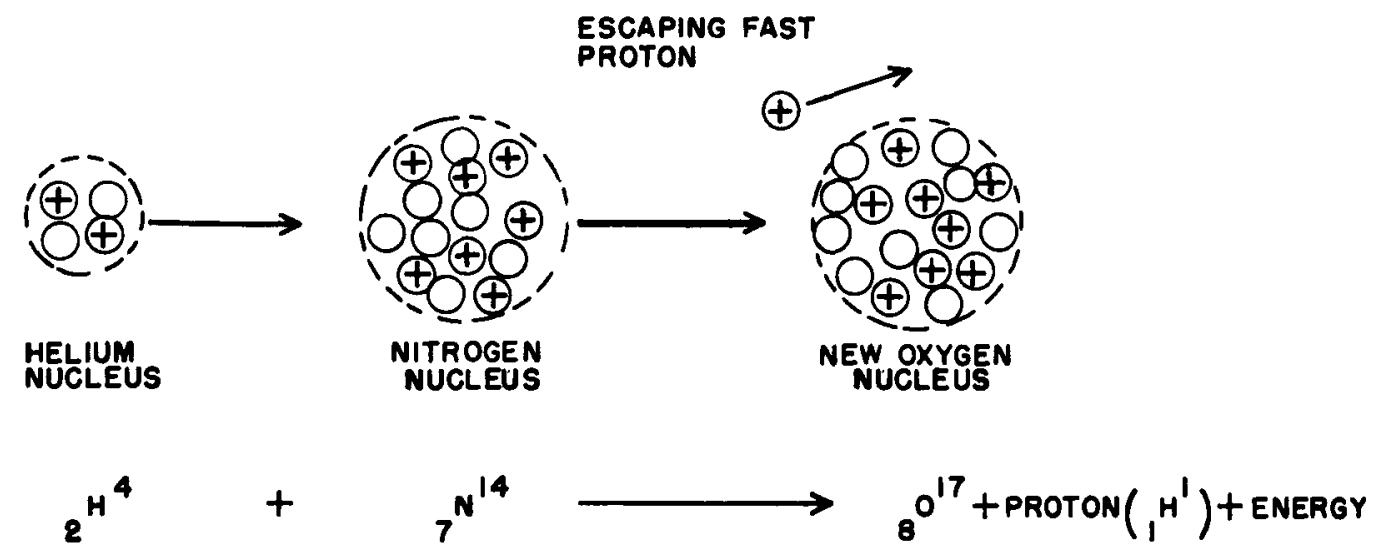

FIGURE 10

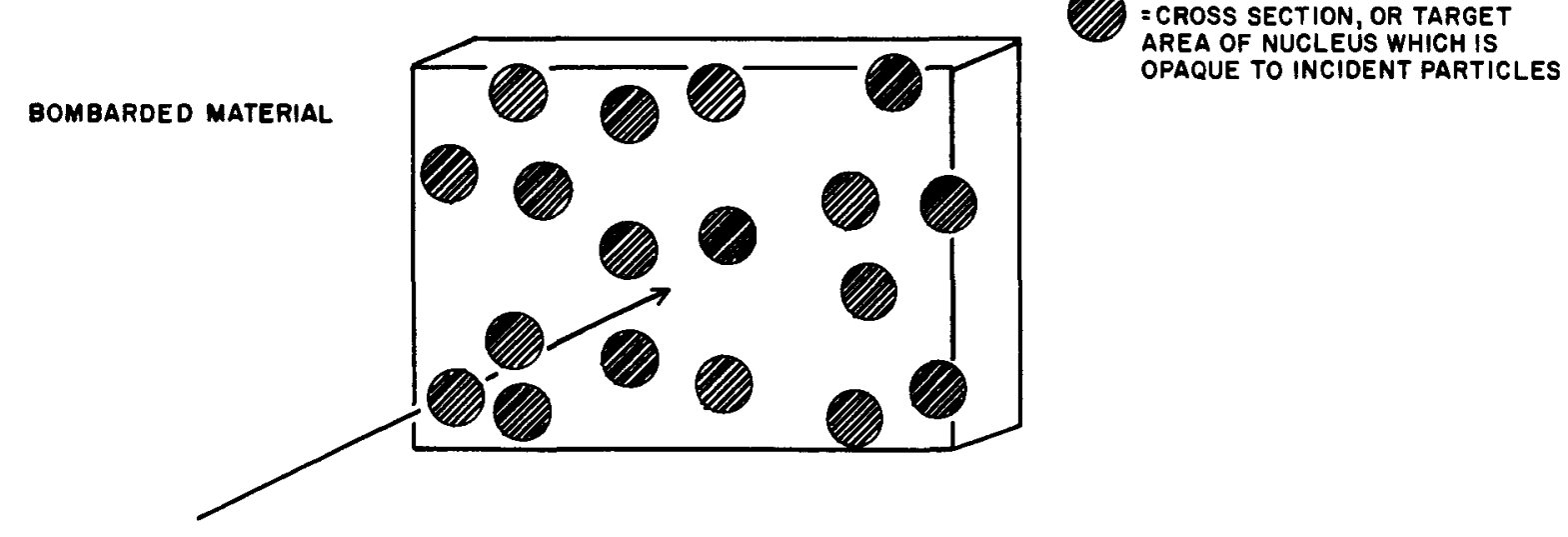

STREAM OF INCIDENT PROJECTILES

THE FRACTION OF THE INCIDENT PROJECTILES ABSORBED BY THE TARGET NUCLEI IS EQUAL TO THE FRACTION OF THE AREA WHICH IS OPAQUE.

FIGURE 11

Cross-Section 
In using this condensed method of writing a nuclear reaction, the subscripts may be omitted because the chemical symbol establishes the value of the atomic number. Thus the subscript six is understood for carbon because this is the only atomic number (and subscript) carbon can have; similarly, the subscript for nitrogen is always seven. The symbols inside the parentheses designate in order the incident and ejected particles, but they are not the chemical symbols used in the longer method. A proton is designated by the latter " $\mathrm{p}$ " instead of ${ }_{1} \mathrm{H}^{l}$. The other symbols used to represent the incident (projectile) and ejected particles are:

$$
\begin{array}{ll}
\mathrm{n}=\text { neutron } & \alpha=\text { alpha particle } \\
\mathrm{d}=\text { deuteron } & \gamma=\text { gamma } \text { ray }
\end{array}
$$

It is common to specify types of nuclear reactions by using the notation inside the bracket. Thus, a reaction in which a deuteron strikes a nucleus and ejects a proton from it is called a $(d, p)$ reaction. Other reactions are represented by $(p, \gamma),(n, p),(n, 2 n),(d, \alpha),(\alpha, p),(\gamma, n)$, and so on.

Mass and Energy Relationships

The study of nuclear reactions is quite similar to the more familiar study of chemical reactions. Here one meets such fundamental concepts as the equation of the reaction, and the process of balancing of the equation with respect to quantities which are conserved in the reaction, and the rates of reaction. For example, the reaction between neutrons and boron $\left({ }_{5} \mathrm{~B}^{10}\right)$ is shown below:

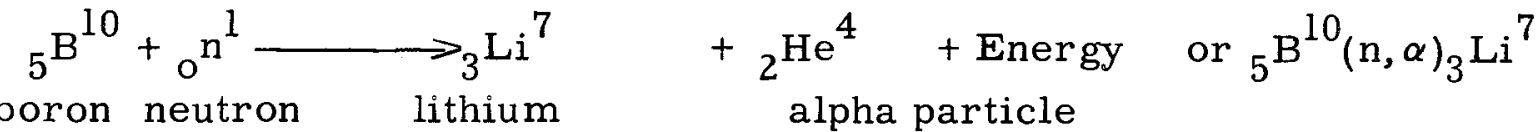

$$
\begin{aligned}
& \text { (This reaction is called an } \mathrm{n}, \alpha \text { reaction) } \\
& \text { neutron alpha }
\end{aligned}
$$


In every nuclear reaction the total number of particles, protons plus neutrons, is conserved. The total electrical charge is also conserved. For example, in the above reaction the balance is as follows:

\begin{tabular}{|c|c|c|}
\hline & LEFT SIDE & RIGHT SIDE \\
\hline Total number of neutrons and protons (mass numbers) & $10+1$ & $7+4$ \\
\hline $\begin{array}{r}\text { Total charge (number of electrons or protons) (atomic } \\
\text { number) }\end{array}$ & $5+0$ & $3+2$ \\
\hline
\end{tabular}

As in the case of chemical reactions, the energy balance of a nuclear reaction provides a measure of the ease with which the reaction can be made to go. In considering energy balances of nuclear reactions one meets a very striking equivalence between mass and energy which is not detected in chemical reactions because of the relatively small energies involved in chem:ical as compared with nuclear processes. This energy can be accurately calculated. If one considers the weight of the interacting nuclei and particles at the beginning of the reactions and the weight of the products, the difference between these two will be represented by the energy change. The energy released in a nuclear reaction results from the fact that the total mass of the final products is less than that of the original particles (see Figure 8).

Mass can be converted into energy and energy into mass. When mass disappears in a nuclear reaction it appears as energy of the reaction. The equivalence between energy in electron-volts and mass in atomic units is set forth in the following relation: (see Table Il)

One unit of atomic weight $=931 \mathrm{Mev}$

or

$1 \mathrm{Mev}=0.00107$ units of atomic weight

Energy equivalent of the mass of an electron $=0.51 \mathrm{Mev}$

As an example of the use of the mass-energy equivalence relation, it will be applied to predict the energy release of the neutron-boron reaction.

UNC LASSIFIED 
The reaction is:

$$
5^{B^{10}}+{ }_{o}^{n^{1}}={ }_{3} \mathrm{Li}^{7}+{ }_{2} \mathrm{He}^{4}+\text { Energy }
$$

We have:

\begin{tabular}{lcccc} 
& \multicolumn{2}{c}{ LEFT SIDE } & & \multicolumn{2}{c}{ RIGHT SIDE } \\
Atomic Weight & (Boron) & 10.01605 & (Lithium) & 7.01804 \\
Atomic Weight & (Neutron) & 1.00893 & (Helium) & 4.00389 \\
Atomic Weight & (Total) & 11.02498 & (Total) & 11.02193
\end{tabular}

Total Atomic Weight (Left Side) 11. 02498

Total Atomic Weight (Right Side) 11.02193

(Difference) 0.00305 units of atomic weight

Therefore, the predicted energy of reaction $=\frac{0.00305}{0.00107}=2.85 \mathrm{Mev}$

The predicted energy release of $2.85 \mathrm{Mev}$ agrees with the experimentally observed energies of the product nuclei.

\section{METHODS OF REACTION}

\section{Projectiles}

The projectiles of nuclear physics are neutrons, protons, deuterons, and alpha particles. These particles are also observed as products of nuclear reactions. In addition the electron or beta particle is a very common product of these processes. Because the electron is not one of the constituent particles of nuclei, it is believed to be created in the course of a nuclear reaction by the conversion of a neutron to a proton plus an electron. In contrast generally to the other particles, electrons are not given off at the instant of the initial reaction but are emitted over a longer period of time in the so-called radioactive beta decay process.

Atomic projectiles can react with the nuclei of the bombarded element in a variety of ways. The most important methods of reaction, excluding the fission reaction which will be described later, are the following: 
1. Impact without capture of the incident particle.

a. Elastic impact

b. Inelastic impact

2. Capture of the incident particle followed by the emission of a nuclear particle (e. g. proton, neutron or alpha particle).

3. Simple capture of the incident particle without emission.

\section{Cross Section}

The chance that a collision between a projectile and a nucleus will result in any one of the types of reactions is expressed in terms of a "cross section" $^{\text {" }}$ of the nucleus. The cross section, actually a concept of probability, is defined as the effective target area which each nucleus presents to the oncoming projectile. If a projectile strikes this "effective area" an interaction with the nucleus is certain.

Atoms have a tiny, close packed nucleus composed of protons and neutrons. The nucleus takes up only one thousandth of one billionth of the volume of an atom, and has a density of more than 100 million tons per cubic centimeter. Therefore, the nucleus, or target, is pictured as a very small area which the projectile must strike. However, physical size is not the only factor to be considered.

In a flight of about three inches through the air an alpha particle passes through about 150,000 molecules or atoms. It is resisted by them almost not at all at first, each atom slowing it up but a trifling amount. But it is slowed down a little, and the more it slows down, the more the atoms resist it, until it is finally brought to a halt. But when a projectile passes through space that is filled only with electrical fields of force (through the electron shells) the chance of a collision depends primarily on direction and velocity.

A comet or meteor or another small planet, if it could be fired through our solar system with sufficient speed, would stand an excellent chance of getting through without actually colliding with anything. The gravitational fields of 
force of every member of the solar system would be tugging at it as it passed through, but the faster it went the less time would it remain in a region where those forces had any appreciable strength, and the less would be their effects upon it. Most of the wanderers that our solar system has picked up from out of space are with us solely because they were not going fast enough. They are caught and forever entangled in the web of gravitation that is woven around us. It is for these reasons that we regard material atoms as things analagous to our solar system in miniature. The "cross section" can be imagined as an opaque (not penetrable) area associated with each individual nucleus. This concept is illustrated in Figure 11, page 35. Fast and Slow Neutrons

The enormous range of neutron velocities calls for descriptive terms. Neutrons which have just been emitted by nuclei have energies of the order of several Mev and are called "fast neutrons". Fast neutrons can scatter inelastically and can also produce neutron-proton $(n, p)$ and neutron-alpha $(n, \alpha)$ reactions. Neutrons which have been slowed into the range of a fraction of an electron volt are called "slow neutrons". Neutrons at in-between energies are called "intermediate neutrons".

Intermediate neutrons are characterized by the phenomenon of resonance absorption, in which neutrons in a narrow energy band a re very strongly absorbed by certain nuclei. Neutrons which have been slowed down by successive impacts to energies approximating the energy of ordinary thermal agitation, (about $0.025 \mathrm{ev}$ ) are called "thermal neutrons". Thermal neutrons are scattered elastically, and are easily absorbed or "captured" by most nuclei by way of the neutron-gamma $(n, \gamma)$ reaction. It will be shown later that on the average, over 100 collisions with carbon nuclei are required to make a thermal neutron from a two Mev fast neutron.

Neutron Reactions

The principal features of the various methods of reaction will now be described, using for purposes of illustration reactions in which neutrons are 
the projectiles. Reactions of neutrons with nuclei differ from reactions with charged projectiles in that the electrically neutral neutron (which contains equal quantities of positive and negative charge) does not experience the repellent force of the charged nucleus. Consequently the neutron reaches and enters the nucleus with greater ease. However, this ease of penetration does not have particular effect on the sequence or types of events which occur after entrance into the nucleus.

Elastic Impact (Without Capture)

In an elastic impact the total kinetic energy (energy of motion) of the neutron and the struck nucleus is conserved. By the term "elastic" in this connection we are referring to rebound from collision rather than from stretching - such as a rubber ball rather than a rubber band. A perfectly elastic ball, as we use the word, is one that would rebound from a floor of some perfectly elastic material back to the height from which it was dropped. For example, consider the collision of two elastic steel balls of the same mass. If one of the balls is initially at rest and the other moving ball strikes it, the total momentum of the system of two balls remains unchanged. Before the impact all the momentum resides in the moving ball; after the impact, this same amount must be found in one or both of them (Figure 12).

In the neutron slowing down reaction, at each collision the neutron gives up a fraction of its energy to the nucleus which it strikes. The lighter this nucleus the greater the fractional energy loss per collision. In collisions with ordinary hydrogen, for example, the neutron loses on the average about 60 per cent of its energy per collision. In collisions with carbon nuclei (heavier), the neutron loses on the average about 15 per cent of its energy per collision. The loss of energy by two Mev neutrons, upon successive collisions with carbon nuclei, is illustrated in the following table:

$\begin{array}{lccccccc}\text { No. of Collisions } & 0 & 20 & 40 & 60 & 80 & 100 & 115 \\ \text { Energy (ev) } & \begin{array}{c}2 \times 10^{6} \\ (2 \mathrm{Mev})\end{array} & 9 \times 10^{4} & 4 \times 10^{3} & 2 \times 10^{2} & 6.6 & 0.26 & 0.025 \\ & & & & & & & \\ \text { (thermal }) & & & & & & \\ & & & & & & & \\ & & & & & & & \end{array}$




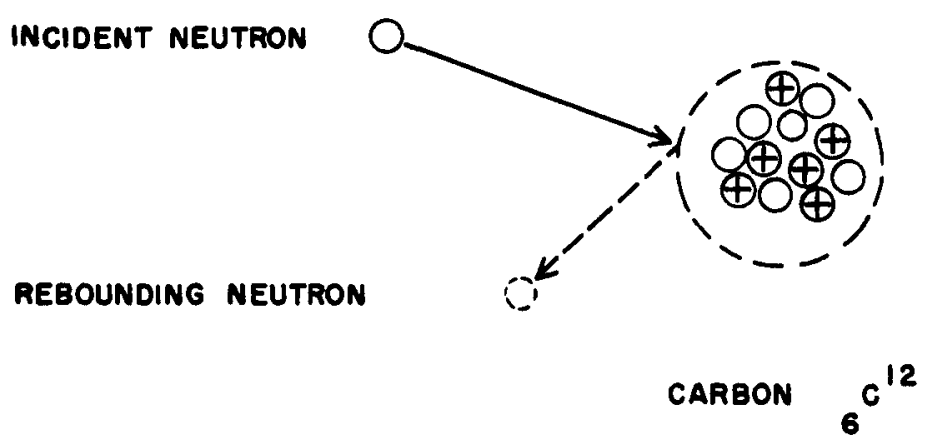

FIGURE 12

Elastic Impact

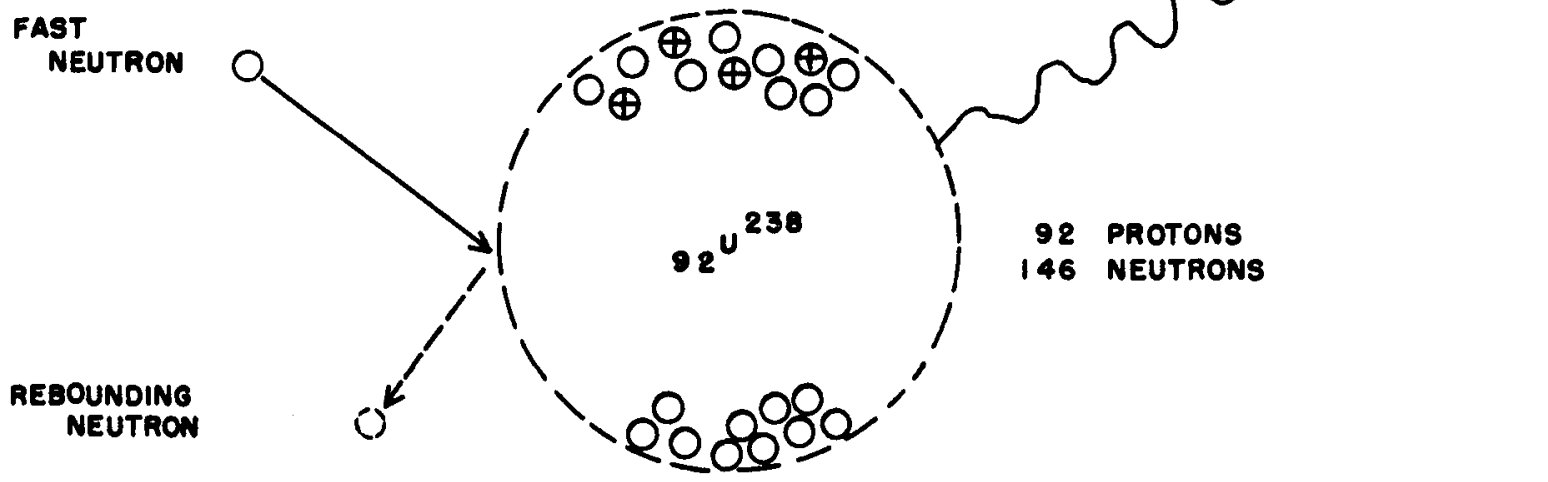

URANIUM

FIGURE 13

Inelastic Impact 
Inelastic Impact (Without Capture)

In an inelastic impact the structure of the bombarded nucleus is sufficiently disturbed so that the excited nucleus emits a radiation to dispose of the energy which it acquired in the collision. The radiation has the form of a ray having wave properties similar to those of X-rays. It is called a gamma ray. Gamma rays behave as if they were particles without mass which have energies proportional to the frequency of the wave radiation. Their energy is usually of the order of several Mev. The emission of gamma rays is a common way for an excited nucleus to dispose of its excess internal energy; gamma rays come from the nucleus and not from the excitation of the planetary electrons, as in the case of X-rays. Their loss alters neither the charge or mass of the nucleus; that is, they come from a shift in the energy level of a given nucleus. The chance that a neutron will make an inelastic collision with a nucleus is hardly ever greater than the chance that it will make an elastic collision. An illustration of inelastic impact is shown in Figure 13. However, "inelastic scattering" is the principal way in which fast neutrons are reduced to intermediate energy by a reactor shield. Capture of Incident Particle with Emission of a Particle

The principal particles emitted in nuclear reactions are protons, alpha particles, beta particles (electrons), and neutrons. In addition, nuclear reactions generally result in gamma ray emission. The reactions shown in Figure 14 illustrate those which eject protons and alpha particles upon neutron bombardment. Reactions in which an incident neutron causes the ejection of two neutrons are also known.

The ease with which a charged particle is ejected depends upon the size of the charge of the target nucleus. With increasing charge the emis sion becomes more difficult. The difficulty arises from the nature of the cohesive forces (binding energy) of the nucleus which seem to become greater as the nuclear charge is increased. In fact, for the heaviest elements the fastest available neutrons are not effective in producing this type of reaction, (excluding the fission reaction). In contrast to the behavior of the very

UNCLASSIFIED 
heavy nuclei, several of the very light elements, for example boron, eject charged particles upon irradiation with neutrons of thermal energies. This behavior of boron upon slow bombardment, combined with its high capture cross section, is the basis for the use of boron in thermal neutron detectors. Simple Capture Without Emission of a Particle

Simple capture is the most common process resulting from neutron bombardment. In this process the incident neutron is absorbed and a gamma ray is emitted. Capture of the neutron results in the formation of an "isotope" of the target element. Whether or not this product nucleus is stable can be predicted by examination of the list of stable isotopes of the target element. For example in Figure 15, aluminum, which is the target, does not have a stable isotope of mass number 28. The product nucleus, ${ }_{13} \mathrm{Al}^{28}$, is unstable (too many neutrons) and gives off energy until stability is attained. In this case the energy is carried off by beta particles and gamma rays. This is an instance of artificially induced radioactivity. The unstable nucleus will tend to acquire stability by a process known as "radioactive decay".

The chance for simple capture is in general very much greater for slow neutrons than for fast neutrons. Because slow neutrons are quite readily captured, they have been found to be the most effective projectiles for use in the quantity production of artificially radioactive materials.

\section{Resonance Capture}

In the bombarding of different target elements with various projectiles, such as protons, neutrons, etc., it has been found that when the incident particles have certain specific energy values, there is a sharp increase in the reaction rate. That is, for certain energy values, the probability that the incident particle will be captured is exceptionally large.

Slow neutrons are generally absorbed more readily than fast neutrons. In other words, the neutron absorption cross section is generally greater for slow neutrons than for fast neutrons. In many elements, notably boron, 

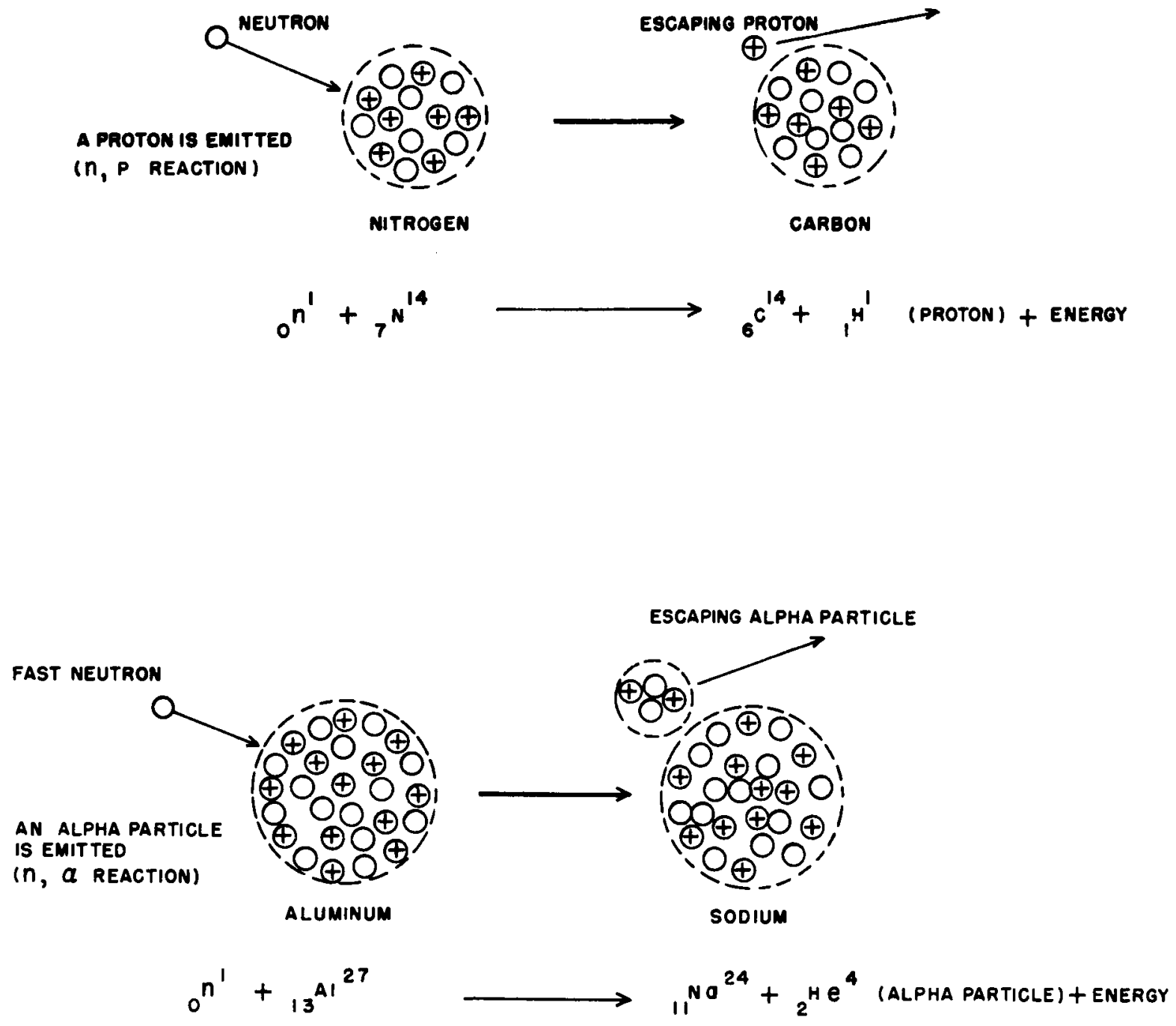

FIGURE 14

Capture of Incident Particle with Emission of a Particle 


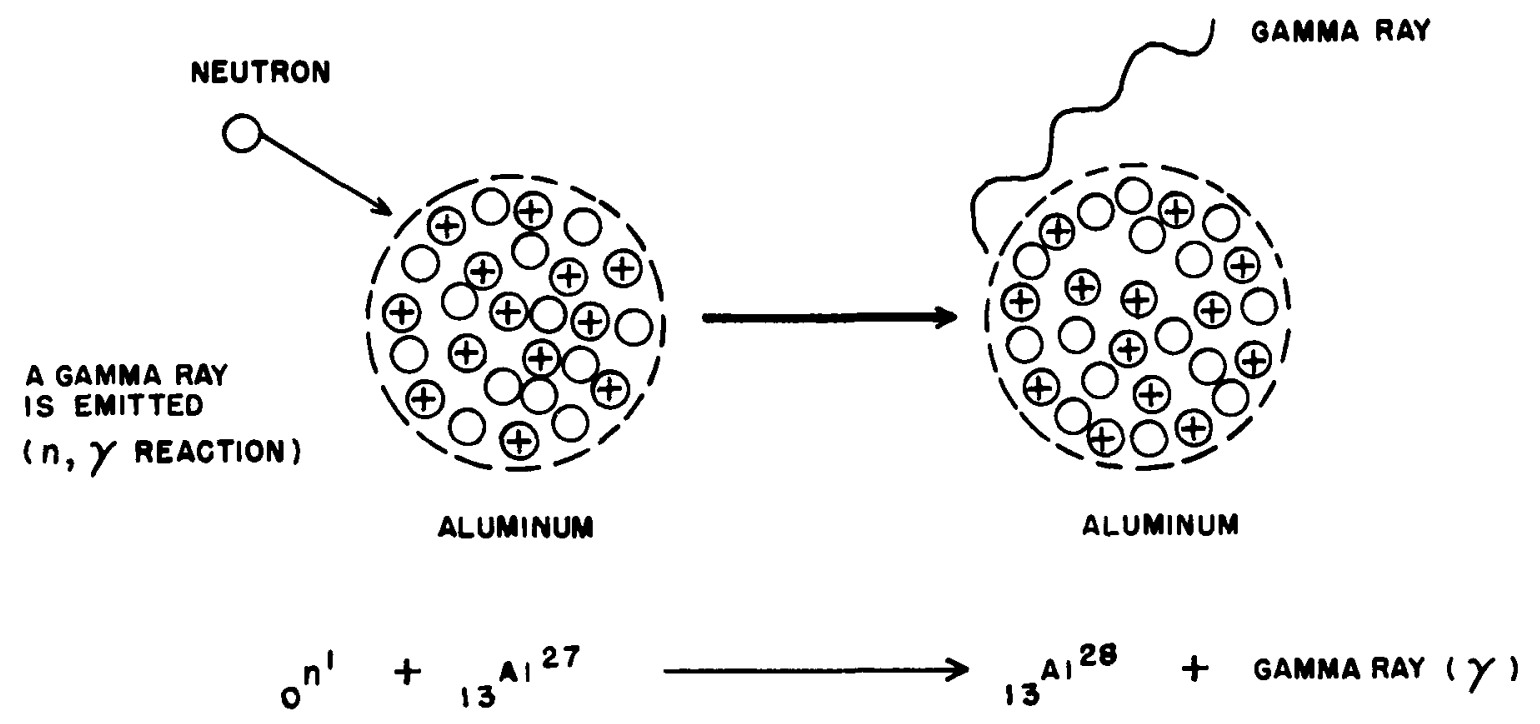

FIGURE 15

Simple Capture Without Emission of a Particle

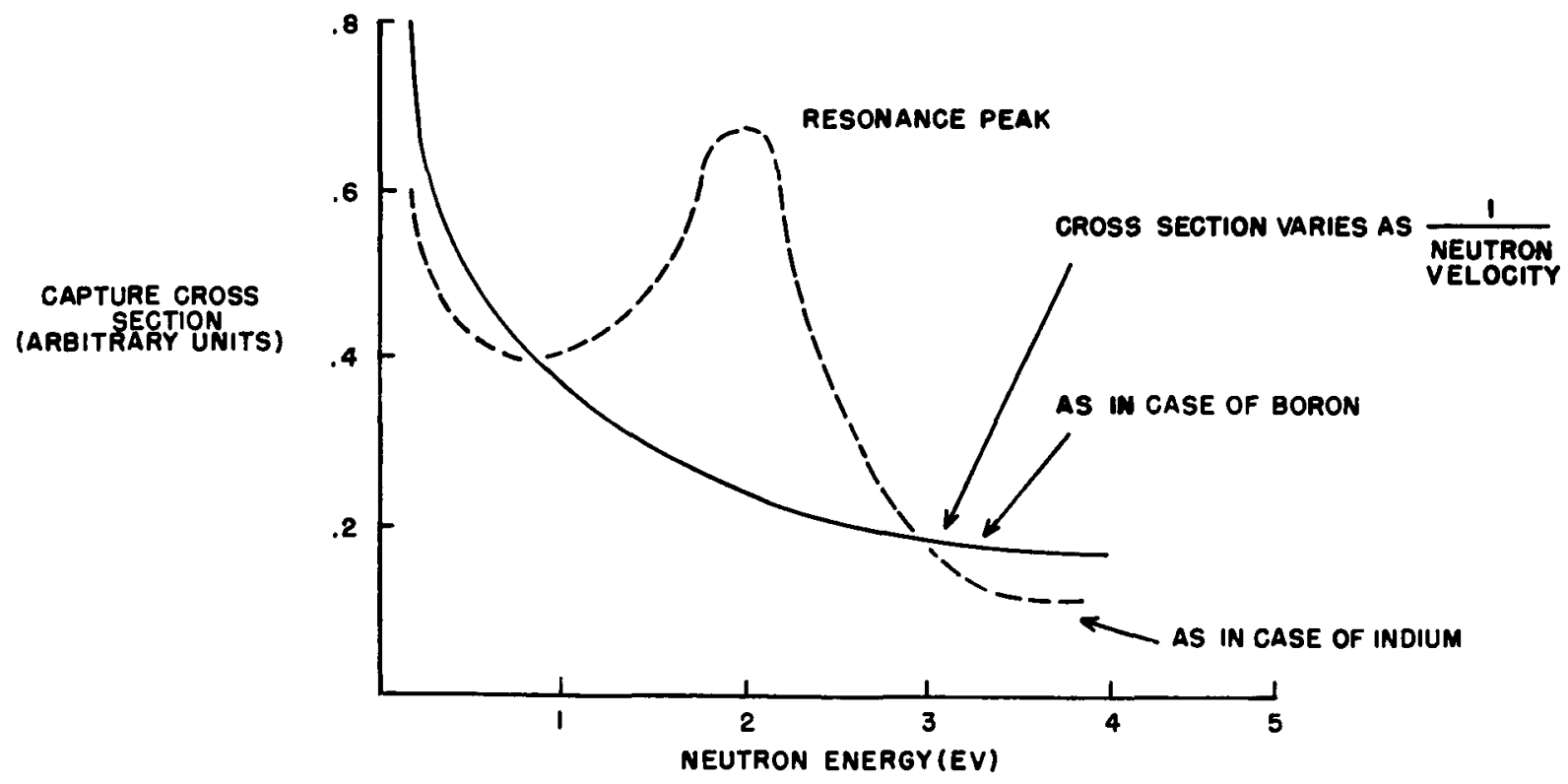

FIGURE 16

Variation of Cross Section with Neutron Energy

MC.eE RICHLAND, wASH. 
the dependence of cross section on neutron velocity is very regular. As the neutron velocity is decreased the nuclear cross section of the boron nucleus increases in an uniform manner, seemingly without bounds as the velocity is decreased further and further.

For these elements the cross section of the nucleus varies inversely proportional to the velocity of the oncoming neutron. Many elements like indium, cadmium, and uranium do not exhibit uniformly increasing cross sections as the neutron velocity is decreased. For these elements the absorption is particularly strong at certain energy levels which are characteristic of the element. This strong capture of neutrons of certain energies is known as "resonance capture". The dependence of cross section on neutron velocity for the two types of absorption is illustrated in Figure 16.

Resonance capture by indium occurs at $1.44 \mathrm{ev}$, for uranium in the region of $25 \mathrm{ev}$. Cadmium owes its usefulness in nuclear physics to the fact that its resonance capture region corresponds to the energy of the thermal neutrons; cadmium can, therefore, be used to remove these slow neutrons from a beam which contains both fast and thermal neutrons.

\section{NUCLEAR FISSION}

$\underline{\text { Neutron Induced Fission }}$

In previous examples of nuclear reactions we have seen where the neutrons or other particles encountering the nucleus caused a reaction that resulted in the emission of gamma radiation or the expulsion of a particle such as an electron (beta activity), alpha particle, etc. But when a free neutron encounters a nucleus of the heavier elements at the end of the periodic table (high atomic numbers), the target nucleus is often split asunder into two parts which are approximately equal. This fissioning or splitting into two may be effected by other particles under the proper circumstances, but as we are concerned with uranium fission, we will confine ourselves to the effect of the neutron on uranium. 
The most important fission is that of the uranium-235 nucleus. In this reaction the uranium captures a neutron and immediately splits into two nuclei. These new nuclei belong to elements in the middle of the atomic series. The importance and great value of this reaction results from two of its features. First, each fission is accompanied by the largest energy release yet obtained from a controlled nuclear reaction. Second, the fission reaction releases neutrons in amounts sufficient to make the fission reaction self-sustaining when the conditions are properly controlled. The reactants and products of fission can be presented in the form:

$$
\begin{aligned}
\text { Uranium nucleus }+ \text { single neutron } \longrightarrow & \text { (two new nuclei) } \\
\text { (several free neutrons) } & \text { (large amount of ener gy) }
\end{aligned}
$$

\section{Liquid Drop Mode1}

In the fission process the uranium nucleus behaves like a liquid drop of matter. The attractive nuclear forces act in the same manner as surface tension of a drop of water (the tendency to pull the surface molecules inward and shrink the surface as small as possible is called surface tension). In opposition to the surface tension are the repellent forces of the 92 similarly charged protons of the nucleus. As one proceeds upward in the atomic scale, the balance between these two opposed forces in favor of stability of the nucleus becomes smaller as the mass number of the nucleus increases and consequently as the volume of the nuclear drop is increased. The shape of the drop is normally spherical because this is the most stable shape. Entrance of a neutron sets the drop into vibration. The sequence of events which follow is illustrated in Figure 17. It is seen that the oscillations may distort the droplet into an unstable dumbbell shape with the end masses approximately equal. Fracture accurs with the formation of two new nuclei and the emission of neutrons.

At the instant of fracture the highly excited nuclear fragments have sufficient energy to eject neutrons. Consider the typical reactions: 
I.<smiles></smiles>

2.<smiles>c1ccccc1</smiles>

3.<smiles>C1#CCCCC#CCCCCC#C1</smiles>

URANIUM NUCLEUS IN NORMAL SPHERICAL SHAPE CAPTURES A NEUTRON.

THE ENEROY IMPARTED TO THE NUCLEUS APPEARS AS MOTION OF THE ENTIRE DROPLET.

VIOLENT OSCILLATION MAY DRAW THE DROPLET INTO A DUMBELL SHAPE.
4.<smiles></smiles>

5.<smiles>C1#CC2(c3ccccc3)CC2CC1</smiles>

6.<smiles>C1=CCCCC1</smiles>

VARIATIONS IN OSCILLATIONS RESULT IN UNEQUAL DISTRIBUTION OF MASS, ELECTRICAL REPULSION ACTS TO PUSH ENDS FARTHER APART.

FRACTURE OCCURS RESULTING IN TWO FISSION FRAGMENTS.

THE FRAGMENTS ATTEMPT TO ACOUIRE STABILITY BY EMISSION OF NEUTRONS.

\section{FISSION FRAGMENTS}

ALTHOUGH THE FISSION FRAGMENTS HAVE VERY ROUGHLY THE SAME MASSES, THEIR WEIGHTS ACTUALLY FALL INTO EITHER OF TWO RATHER DISTINCT MASS RANGES. THE TWO MASS RANGES ARE PRESENTED IN THE FOLLOWING FIGUAE - 18.

FIGURE 17

Fission of Uranium

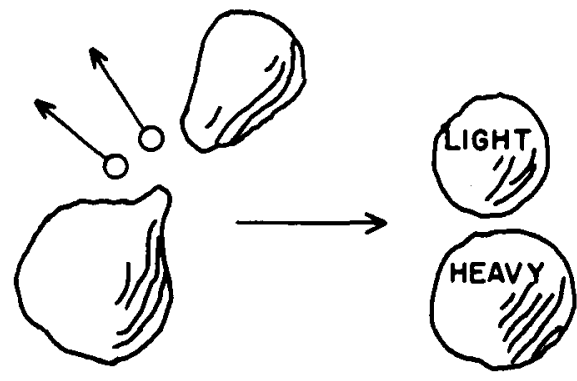

RANGE IN ATOMIC WEIGHT 85 - 104 INCLUSIVE

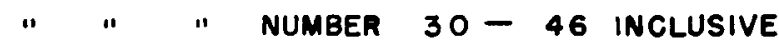

RANGE IN ATOMIC WEIGHT $130-149$ INCLUSIVE " " NUMBER 46 - 62 INCLUSIVE

"LIGHT" gROUP INCludes ZINC, gallium, germanium - to palladium, inclusive "heAvY" GROUP INCludes PALladium, ARGENTUM, CADIUM - TO SAMARIUM

FIGURE 18

Mass Ranges 
${ }_{92} \mathrm{U}^{235}+{ }_{\mathrm{o}^{\mathrm{n}^{1}}}$ (slow) $\longrightarrow{ }_{92} \mathrm{U}^{236} \underset{\text { highly excited }}{\longrightarrow}{ }_{36^{\mathrm{Kr}}}{ }^{94}+\underset{\text { highly excited }}{56^{\mathrm{Ba}}}$

In every nuclear reaction the total number of particles, protons plus neutrons, is conserved. The total electrical charge is also conserved. In the above reaction the balance is as follows:

Total number of neutrons and protons
(mass number)

LEFT SIDE RIGHT SIDE $235+1 \quad 94+142$

Total Charge (Atomic Number)

$92+0 \quad 36+56$

On the average, between two and three fast neutrons are emitted by the excited nuclei immediately on fission. The emission of these several neutrons in place of the single neutron captured makes self-sustained fission possible. To illustrate the emission of the fast neutrons we have:

${ }_{36} \mathrm{Kr}^{94}+{ }_{56} \mathrm{Ba}^{142} \longrightarrow \underset{\text { Unstable }}{36^{\mathrm{Kr}}}{ }^{92}+\underset{\text { Unstable }}{56^{\mathrm{Ba}^{141}}+3 \mathrm{o}^{1}+\text { Energy }}$

In this reaction three neutrons are emitted, two neutrons from the krypton nucleus and one neutron from the barium nucleus. Let's balance the sides:

Total Number of Neutrons and Protons

$\begin{array}{ll}\frac{\text { LEFT SIDE }}{94+142} & \frac{\text { RIGHT SIDE }}{92+141+3} \\ 36+56 & 36+56\end{array}$

Total Charge

As indicated in the equation, the products are unstable. This results from the unsatisfactory neutron to proton ratio of these nuclei. The unstable krypton and barium nuclei will decay radioactively with beta ray emission, by converting neutrons to protons, in a so-called "fission chain". 
The decay will continue from element to element until a stable isotope of the same mass number as the original unstable nucleus is reached. For example, the above unstable nuclei will decay with beta and gamma emission in the following possible fission chain:

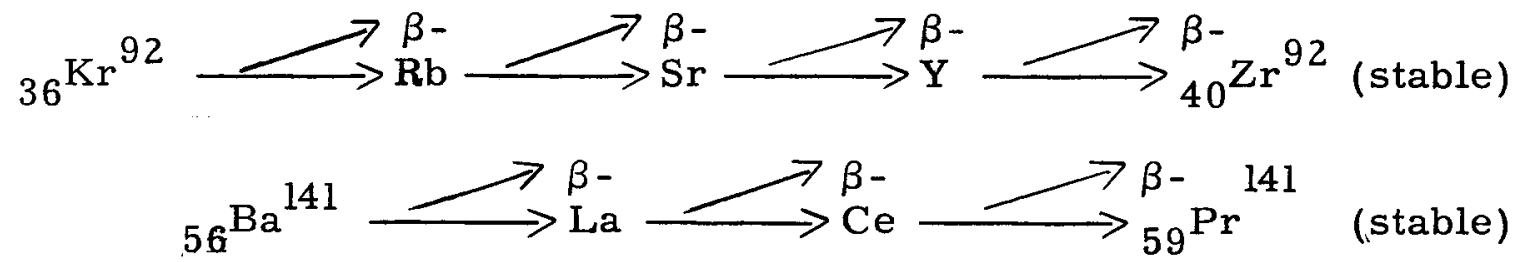

In the case of krypton, four neutrons were converted to protons - atomic number was raised from 36 to 40 .

In the case of barium, three neutrons were converted to protons the atomic number was raised from 56 to 59 .

\section{Energy Release}

The tremendous energy released in the fission process results from the fact that the total mass of the final stable products is less than that of the original particles. In accordance with the mass-energy equivalence (see Figure 8, page 28), the difference in mass between the original particles and the final stable products will be represented by the energy change. An estimate of the magnitude of the energy released per fission can be obtained by consideration of the reaction already referred to:

${ }_{92} \mathrm{U}^{235}+\mathrm{o}^{\mathrm{n}^{1} \longrightarrow} \longrightarrow \begin{aligned} & \text { (fission and neutron } \\ & \text { emission followed by } \\ & \text { decay to stable products) }\end{aligned}{ }_{40} \mathrm{Zr}^{92}+{ }_{59} \mathrm{Pr}^{141}+{ }_{0} \mathrm{n}^{1}$

To compute the amount of mass which is converted to energy, the difference in the sums of the atomic weights is obtained. 
Atomic Weight of Original Particles

$$
\mathrm{o}^{\mathrm{n}}
$$$$
92^{\mathrm{U}^{235}}
$$

Atomic Weight of Final Particles

$$
\begin{aligned}
& 40^{\mathrm{Zr}^{92}} \\
& 59^{\mathrm{Pr}^{141}} \\
& \mathrm{o}^{\mathrm{n}^{1}}(3)
\end{aligned}
$$$$
\text { 91. } 9420
$$$$
\text { 140. } 9590
$$

Mass Difference $=0.205$ Units

Energy released per fission $=\frac{0.205 \mathrm{amu}}{0.00107 \mathrm{amu} / \mathrm{Mev}}=191.6 \mathrm{Mev}$

The estimated energy release is close to the accepted average release of $200 \mathrm{Mev}$ per fission. Most of the energy appears as energy of motion of the large fission fragments. This energy is dissipated by the heating of the stopping materials. The remainder of the energy appears as energy of the gamma rays, beta particles and neutrons which accompany this process. The distribution of the energy among the various particles is as follows:

\section{Distribution of Fission Energy}

Kinetic energy of fission fragments

\begin{tabular}{c}
$\frac{\text { Mev }}{168 \pm 5}$ \\
$5 \pm 1$ \\
$7 \pm 1$ \\
$5 \pm 0.5$ \\
$6 \pm 1$ \\
10 \\
\hline
\end{tabular}

Total Fission Energy

$201 \pm 6$ 
All the energy released within the reactor, disregarding the neutrino energy, is ultimately transformed into heat; the various mechanisms whereby this takes place are as follows:

1. Transfer of kinetic energy from fission products.

2. Transfer of kinetic energy from fission neutrons.

3. Deceleration and absorption of beta particles.

4. Absorption of gamma rays.

\section{Prompt Neutrons}

The neutrons emitted as a result of the fission process can be divided into two categories, namely "prompt neutrons" and "delayed neutrons". The prompt neutrons, which are over 99 per cent of the total fission neutrons, are released within an extremely short interval of time, about $10^{-14} \mathrm{sec}$, following the fission process. It appears that the prompt neutrons are emitted at the instant of fracture; the two nuclear fragments, each of which has too many neutrons for stability, expels one or more neutrons within a very short time after its formation. The instantaneous gamma rays accompanying fission are apparently emitted at the same time.

\section{Delayed Neutrons}

Whereas the expulsion of the prompt neutrons is accomplished within a very short time, the delayed neutrons are emitted, with gradually decreasing intensity, over a period of minutes. Bromine and iodine have been identified as the source of two of the neutron activities. At least three other activities are present but their sources have not been identified. By observing the decay of the delayed neutrons after fission has ceased, it has been possible to associate a specific half-life with each group. By making rapid chemical separations of the fission fragments, it has been found that the emission of delayed neutrons does occur in an indirect manner from bromine and iodine isotopes. 
One of the products of fission is a bromine isotope of high mass number, probably $\mathrm{Br}^{87}$, the nucleus of which contains too many neutrons for stability and is consequently a beta emitter. The half-life of $\mathrm{Br}^{87}$ is 55. $6 \mathrm{sec}$, which is the same as the half-life of one of the groups of delayed neutrons, and its decay product is $\mathrm{Kr}^{87} \cdot \mathrm{Kr}^{87}$ can evidently be formed in a highly excited state, with sufficient energy to immediately eject a neutron and leave a stable $\mathrm{Kr}^{86}$ nucleus (Figure 19). The observed rate of emission of neutrons is thus determined by the rate of formation of the neutron emitter $\mathrm{Kr}^{87}$, and this is dependent on the decay of the precursor (parent) $\mathrm{Br}^{87}$. Like all radioactive elements, the latter decays in an exponential manner, the half-life in this case being $55.6 \mathrm{sec}$, so the neutron emission falls off at the same rate.

The precursor of the group of delayed neutrons with a half-life of $22.5 \mathrm{sec}$ is apparently iodine $\left(\mathrm{I}^{137}\right)$. It is known to have a half-life of 22.5 sec and when it decays, by the emission of a beta particle, the product is xenon $\left(\mathrm{Xe}^{137}\right.$ ). The $\mathrm{Xe}^{137}$ is in a state of high internal energy so that it instantaneously expels a neutron to form stable $\mathrm{Xe}^{136}$. Here again the emission of the neutron is delayed because the rate of formation of the $\mathrm{Xe}^{137}$ from which it originates depends on the rate of decay of the precursor $\mathrm{I}^{137}$. The other three groups of delayed neutrons are produced in a similar manner, although the parent particles in these cases have not yet been definitely identified.

VIII. DETECTION OF NUCLEAR RADIATION

Ionization

The detection of nuclear particles is usually accomplished by detection of the ionization which they produce. Ionization is the process by which moving, charged particles dislodge electrons from the atoms of the material through which they pass. Removal of the electron from a neutral atom leaves a relatively massive ion. The detection of these ions and free electrons by electrical means constitutes the detection of the 
particle (Figure 20). The alpha particle and proton ionize very strongly, the electron and positron weakly. The electrically neutral neutron and the gamma ray produce litttle ionization. Their detection depends principally on the measurement of ionization produced by secondary effects, caused by reactions of the radiation with atomic electrons.

Detection

The simplest detector of ionization by the nuclear radiations is the Lauristen electroscope. In principle it is the same as the gold-leaf electroscope in which the presence of charge on the leaves is indicated by their separation. This principle is used in personnel pencil meters used routinely at atomic plants. Deflection of the leaves indicates the presence of ionization: the rate of deflection is a measure of the intensity of the ionizing radiation. The Lauristen electroscope has been made very sensitive by the substitution of a very fine, metallized, quartz fiber for the heavy leaf.

For the detection of a very small number of particles or for more precise measurements of beta or gamma ray intensities, the Geiger-Mueller tube counter is used. The tube counter is used in conjunction with an electronic amplifier and recording unit. Cumulative ionization in the counting tube results in sharp current pulses upon passage of single particles through the tube gas. These pulses are amplified and recorded by the accessory equipment. This principle is used in the proportional counter (PC).

Neutrons, having no charge, produce no direct effects on electrical counting instruments. They are detected by their secondary effects. A common method is to cause the neutrons to eject alpha particles from boron. The ionization by the resultant alpha particle is relatively easy to detect and measure. Another important method for the detection of neutrons involves the measurement of the activity induced in a suitable detecting foil by neutrons being measured. The electron emission of the activated foil is measured by means of a Geiger-Mueller tube counter or the Lauristen electroscope, an ion chamber, or a scintillation counter.

UNCLASSIFIED 


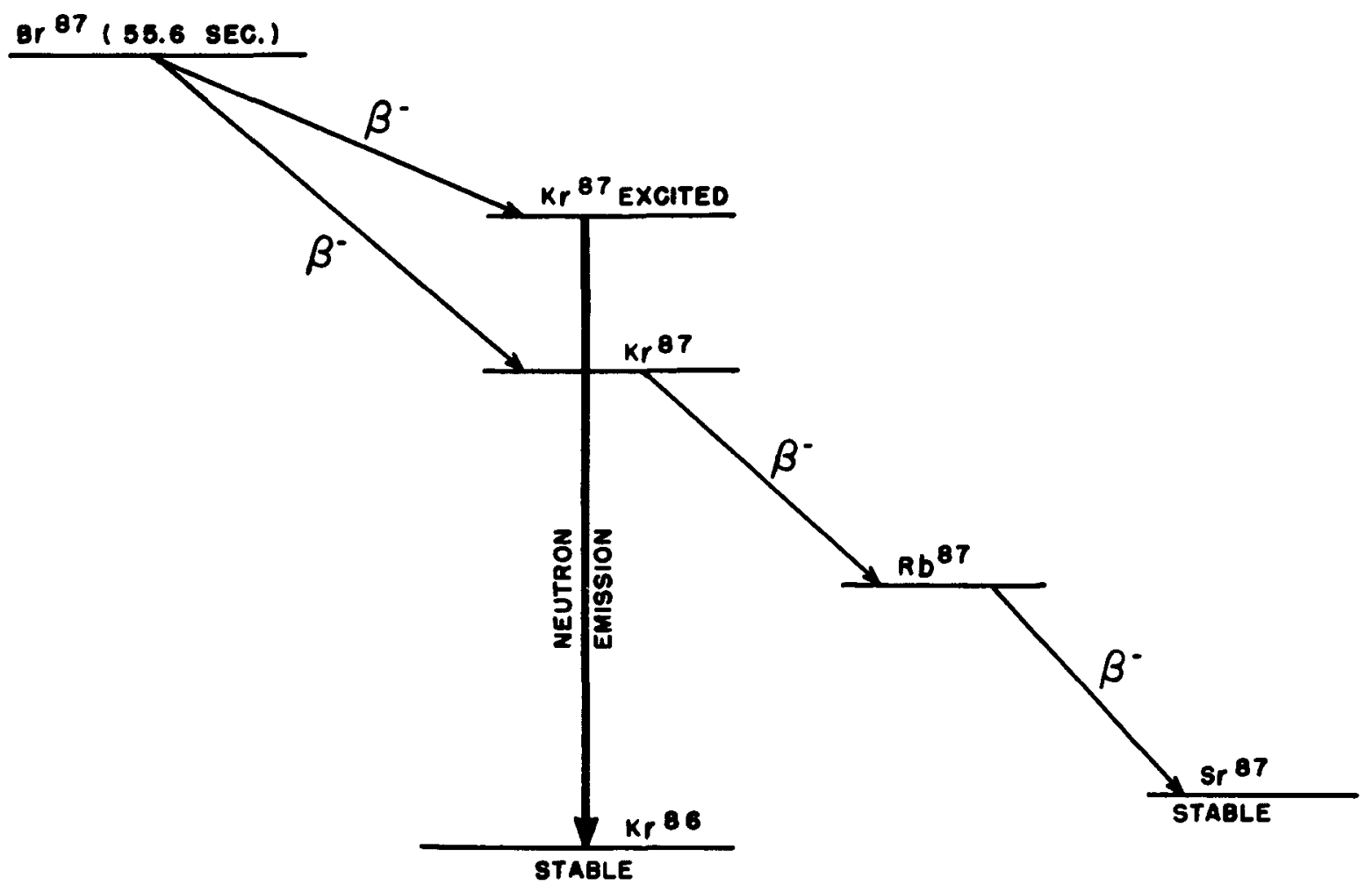

FIGURE 19

Interpretation of Delayed Neutron Emission

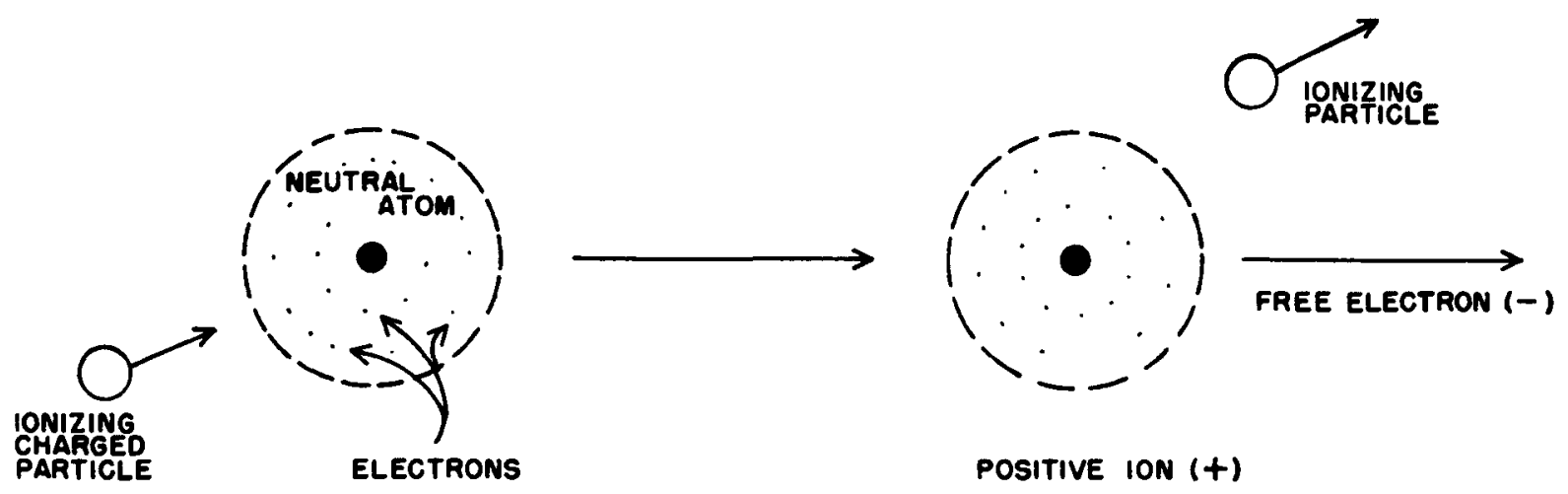

FIGURE 20

Ioni zation 
The detection of gamma rays depend upon recording the ionization produced by the secondary electrons which the gamma rays eject from the walls of the detecting chamber. Because the ionizing particles are electrons, the same detection instruments, for example, the Lauristen electroscope or the Geiger-Mueller tube counter can be used as in the case of beta particles. If the walls of the detecting chamber are made fairly thick and of a heavy element, the probability of ejection of secondary electrons from the walls is greater and, therefore, the efficiency of detection is improved.

Nature of Radiations

In order of increasing hazard, the radiations of interest are alpha particles, beta rays, gamma rays, and neutrons. These radiations can be detected only by instruments, such as Geiger counters, ionization chambers, photographic films, or, at relatively high intensities by fluorescent screens. Without such instruments a man can easily receive a fatal dose without being aware of it at the time. Consequently, an active program of radiation monitoring is highly important. The nature and penetrating power of the radiations of interest are explained below.

\section{Alpha Particles}

The alpha particle and proton have well defined paths in matter and possess a definite range, or length of travel through the matter, which is proportional to their initial energies. These particles lose their energy entirely by ionization. Alpha particles are helium nuclei of mass four and positive charge two. They are ejected simultaneously by the nuclei of uranium and plutonium, but not by the fission elements. Because of their large mass and charge, the penetrating power of alphas is very low. For example, the alphas from uranium are stopped completely by about one inch of air, 0.001 inches of aluminum, or 0.0001 inches of lead. Alphas therefore present a hazard only when bare alpha-emitting metal is handled or when contaminated dust is present. 
$\underline{\text { Beta Rays }}$

Electrons also lose their energy by ionization, but at a slower rate than protons or alpha particles of the same energy. Their ranges are consequently much longer. Because of the light weight of the electrons (beta particles), they are easily deflected by nuclei with the result that their range is not as definite as for the heavier particles. Beta rays are fast electrons of very small mass, roughly $1 / 1800$ that of a proton or 1/7200 that of an alpha particle. They carry a negative charge of one unit and are emitted, with energies ranging up to three or four Mev, by radioactive nuclei. Their penetrating power is rather low; two Mev betas are completely stopped by 23 feet of air or about $1 / 8$ inch of aluminum. Consequently, betas are dangerous only when the observer is directly exposed at a short distance from the source, or again if the source consists of dust-like particles which are easily spread.

Gamma Rays

Gamma rays, also called photons or quanta, are electromagnetic waves or particles whose wave length is roughly one millionth the wave length of visible light. The hardest gamma radiation emitted by a reactor has an energy of about five Mev, whereas the average is probably about two Mev. Unlike alphas and betas, gammas are not easily stopped by matter. Instead, their intensity decreases at a much lower exponential rate as they penetrate matter. The intensity of a beam of two Mev gamma is reduced tenfold by passage through 1300 feet of air, 20 inches of water, 10 inches of concrete, eight inches of aluminum, or 1.7 inches of lead. The gamma radiation from slugs of active metal and from the activity induced in pile water and control rods is highly dangerous and requires massive shielding.

\section{Neutrons}

Neutrons are particles of unit mass and zero charge which are produced in the fission process and are present in quantity only when the pile is in operation. Their penetrating power is considerable and their 
interaction with matter is highly complex. A fast fission neutron of about two Mev energy is first slowed down by numerous collisions with atomic nuclei until it arrives at thermal energy (about $0.025 \mathrm{ev}$ ). Then the thermal neutron continues to bounce around until it is captured by a nucleus either to induce fission or form a heavier nucleus. When the latter happens, a quantity of gamma radiation equal to the binding energy of the neutron in the nucleus is emitted. In many cases, the nucleus is made radioactive by this neutron capture, and later disintegrates to a stable nucleus with the emission or assorted betas and gammas. Neutron shields must therefore not only slow down and absorb neutrons, they must also take care of the gamma radiation, both immediate and delayed, which the neutrons produce. Fast neutrons are reduced tenfold in intensity by passage through approximately four inches of water, 15 inches of graphite, or 17 inches of ordinary concrete.

The physiological effects of radiation arise from their ionizing ability。 Because of their greater penetrating power, fast neutrons and gamma rays present the greatest hazard from exterior sources to the body tissue of the worker. However, the effect on surface tissue of the less penetrating beta particles must not be minimized. A source of radiation ingested into the body which gives off alpha particles or beta particles directly is more harmful than is a gamma source, because the radiation from these particles will be absorbed within such a small volume that the local rate of tissue damage becomes excessive. 


\section{SECTION B}

\section{PHYSICAL PRINCIPLES OF THE REACTOR PROCESS}

\section{DESCRIPTION OF THE REACTIONS}

$\underline{\text { Discovery of Plutonium }}$

In the early months of 1941, scientific deduction and investigation had brought to light a new source of atomic energy which would be produced as a by-product of an atomic pile.

It was then known that when U-238 absorbed a neutron it became U-239 (having added one unit of weight by its captured neutron), but was not happy in this state. In a matter of minutes it underwent beta decay and relieved itself of an electron from its nucleus.

No atom could throw off a nuclear electron and still maintain the same identity. The loss of a negative electron from the nucleus had the effect of changing a neutron to a proton, thus raising by one the positive charge of the nucleus, and permanently annexing a stray electron for the outer electronic shell. As the atomic number of an element changes with its nuclear charge, a nucleus which gained a charge would go up one in atomic number. Hence, where formerly there was a $\mathrm{U}^{239}$ of atomic number 92 , there would now be an $\mathrm{x}^{239}$ of atomic number 93 .

What was this X? In 1940 two American scientists hunted it down among their test tubes. They named it neptunium ( $\mathrm{Np}$ ) because, just as Neptune lies beyond Uranus in the solar system, so this new element lies beyond uranium in the atomic table. This was the first instance of the creation of a transuranic element (one which lies beyond uranium and does not exist in nature). But that is not where the importance of neptunium chiefly lay. Hardly had this new element been created when it was found that it was not a stable element, but released a beta particle 
from ech of its nuclei at such a rate that every 2-1/2 days half of its nuclei were transformed; the loss of a nuclear electron by the $\mathrm{Np}^{239}$ atom of atomic number 93 would change it into an atom of $x^{\prime 239}$ of atomic number 94 . Now the question was: What was $\mathrm{X}^{\prime}-$ ?

This time four American scientists teamed together to get the answer. This second transuranic element, they found, had a relatively stable nature. Like all elements beyond lead it was not completely happy about its nuclear make-up and was radioactive, but was contented enough to emit alpha particles at such a slow rate that it would take 24,000 years for half of it to change. Like the previous elements, the new element was called plutonium $\left(94 \mathrm{Pu}^{239}\right.$ ) after the planet Pluto, which lies beyond Neptune.

Plutonium was an exciting find. Like $\mathrm{U}^{235}$ it had the sort of arrangement of neutrons and protons which physicists had learned to associate with possible fission. Being an element with a half-life of 24,000 years, it had good working stability. As a transuranic element born from the fissionwise inert $\mathrm{U}^{238}$ it was a means for increasing the supply of fissionable material 150-fold. But most important of all, being a different element than uranium it could be separated from the neutron-neutralizing $\mathrm{U}^{238}$ by relatively simple chemical methods. This would make it more accessible than $\mathrm{U}^{235}$, which, having the identical chemical properties of its more abundant isotope $\left(U^{238}\right)$, could only be separated from the other by elaborate and expensive physical means. Thus there was an incentive for the production of plutonium by the reactor process.

Fundamental Nuclear Reactions

Basically, the plutonium production process consists of two types of nuclear reactions. Using the principle that mass and energy are interchangeable we can take advantage of the "binding energy" of different nuclei by either splitting them (fission), combining them (fusion), or adding to and changing them (transmutation) so as to end up with less mass, different elements, and more energy. The production reactor process consists 
of the production of isotopes (plutonium) in some of the atoms and the fission of other atoms. Both types of reaction require neutrons to collide with atoms - to combine with the target nucleus in the first case to form plutonium - to upset the target nucleus and cause a disruption in the second case. Although the neutron is lost to the over-all process in the first case, the disruption of the fissioning atoms causes the release of two or more free neutrons, giving us back at least two for one. Thus we can get a supply of neutrons to keep the reaction going and we will have enough spare neutrons left over to produce isotopes or new atoms in the non-fissioning atoms.

Formation of Plutonium

Plutonium is formed from uranium by nuclear reactions involving the two principal isotopes of this metal as found in nature. These isotopes and their relative abundance are:

$\begin{array}{lcc}\frac{\text { Isotope }}{9 \mathrm{U}^{238}} & \frac{\text { Abundance }}{9.28 \%} & \text { Relative Abundance } \\ 92{ }_{92} \mathrm{U}^{235} & 0.71 \% & 1\end{array}$

The isotope ${ }_{92} \mathrm{U}^{234}$ which is present to the extent of 0.006 per cent in natural uranium is not important in the reactor process.

The fundamental reactions of the process involve capture of neutrons by ${ }_{92} \mathrm{U}^{238}$ and ${ }_{92} \mathrm{U}^{235}$. However, the nuclear reactions which are initiated by neutron capture by these isotopes are entirely different. This difference in behavior of ${ }_{92} \mathrm{U}^{238}$ and ${ }_{92} \mathrm{U}^{235}$ upon neutron capture is the basis of the natural uranium reactor process.

Neutron Capture by $\mathrm{U}^{238}$

When a high energy neutron - one which travels at high speed encounters a nucleus of ${ }_{92} \mathrm{U}^{238}$, it causes fission of the nucleus. A low energy neutron - one which travels at low speed - will be subject to radiative capture. It is mainly the effect of the low energy neutron on 
${ }_{92} \mathrm{U}^{238}$ that interests us; the number of fissions caused by fast neutrons reacting with uranium -238 is only a small fraction of the total number of fissions taking place in the reactor. The capture of a neutron by ${ }_{92} \mathrm{U}^{238}$ leads to the formation of plutonium. The initial reaction is one of simple capture in which an unstable isotope of uranium is formed and a gamma ray is emitted. The reaction can be written:

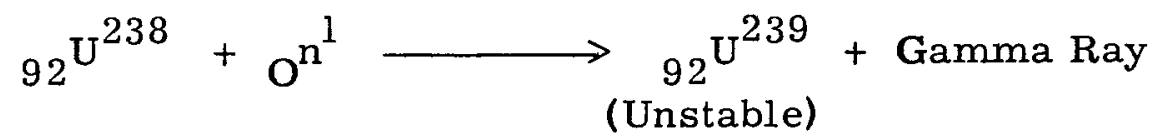

In the reaction above, ${ }_{92} \mathrm{U}^{238}$ plus a neutron gives ${ }_{92} \mathrm{U}^{239}$ plus gamma radiation. But the ${ }_{92} \mathrm{U}^{239}$ has a very short half-life of only 23 minutes; so the newly formed radioactive isotope of uranium decays radioactively, with beta particle emission, thereby forming a different element with an atomic number one greater (see below). The decay, which proceeds with a half-life of 23 minutes, can be written:

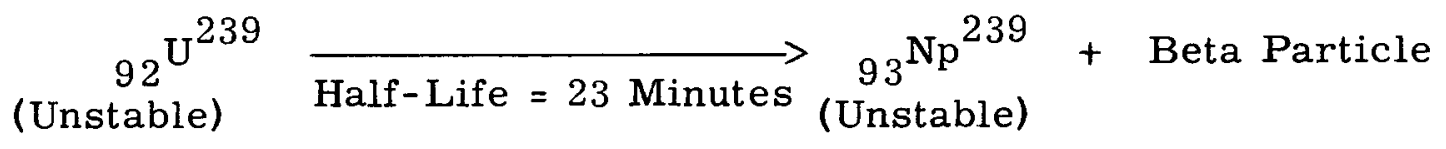

In this reaction $92^{239}$ gives beta radiation plus neptunium $\mathrm{Np}^{239}$. Here we see that $92 \mathrm{U}^{239}$ ejects a negative electron to become neptunium239 , another element and, like its parent, a radioactive isotope. The decay of ${ }_{93} \mathrm{~Np}^{239}$ results in the formation of product, ${ }_{94} \mathrm{Pu}^{239}$. The decay, which proceeds with a half-life of 2.3 days, is described by the reaction:

\section{(Unstable)}

$93^{\mathrm{Np}^{239}} \underset{\text { Half-Life }=2.3 \text { days }}{\longrightarrow} 94^{\mathrm{Pu}^{239}}+$ Beta Particle + Gamma Rays

Here again the product $94 \mathrm{Pu}^{239}$ is the result of the ejection of a negative electron, and also the very rare isotope is formed. It can be created by the nuclear reaction of a slow neutron on ${ }_{92} \mathrm{U}^{238}$, which is the isotope forming the vast majority of natural uranium (99. 28 per cent). Further, unlike its parent $92 \mathrm{U}^{238}$, either a fast or slow neutron can cause fission in plutonium ${ }_{94} \mathrm{Pu}^{239}$.

UNCLASSIFIED 
The nuclear processes which are initiated by the capture of neutrons by ${ }_{92} \mathrm{U}^{238}$ and result in the formation of plutonium ${ }_{94} \mathrm{Pu}^{239}$ are summarized schematically in Figure 21.

Fission of $\mathrm{U}^{235}$

The only naturally-occurring "nuclear fuel" is ${ }_{92} \mathrm{U}^{235}$, which exists in natural uranium to the extent of I part in 140. Capture of a neutron by ${ }_{92} \mathrm{U}^{235}$ results in the fission (splitting apart) of this nucleus. The value of this fission reaction to the over-all production process lies in the fact that it provides a source of neutrons for the ${ }_{92} \mathrm{U}^{238}$ reaction just described. The fission process produces two or more newly formed nuclei (fission fragments) having about $168 \mathrm{Mev}$ of kinetic energy (energy of motion), a fact of great value for power production but a source of engineering limitations to the "production-only" reactor. In addition, the process gives two or three new neutrons which may cause further fission. Thus, a single initiating neutron causes the release of several free neutrons. The reaction, therefore, acts as a neutron multiplier or source. The fission reaction can be presented as follows:

FISSION OF $\mathrm{U}^{235}$ - PROBABLE PRODUCTS

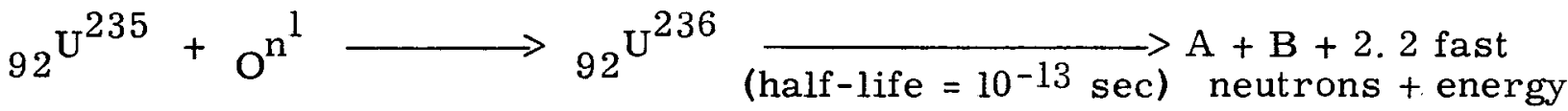

$A=$ light fission fragments in atomic mass range 85-104, inclusive, and in atomic number range $30-46$, inclusive.

$\mathrm{B}=$ heavy fission fragments in atomic mass range 130-149, inclusive, and atomic number range 46-62, inclusive.

Energy released per fission $=200 \mathrm{Mev}$.

Average energy of liberated neutron $=2 \mathrm{Mev}$. 
The ${ }_{92} \mathrm{U}^{236}$ formed by the capture of the neutron disintegrates instantly $\left(10^{-13}\right.$ to $\left.10^{-7} \mathrm{sec}\right)$, releasing a total fission energy of $200 \mathrm{Mev}$. Of this, about 84 per cent, or $168 \mathrm{Mev}$, is dissipated in the kinetic energy of the two fission fragments. These fly apart at speeds nearly $1 / 10$ that of light - equivalent to vibration speeds caused by temperature of several billion degrees.

Fission Products

The discovery of fission was made as a result of the detection of elements of moderate atomic weight, such as barium 56 (atomic number 56 ) and lanthanum 57, when uranium was bombarded by slow neutrons. Since lanthanum has an atomic number of 57 , whereas that of uranium is 92 , the other fission fragment in this reaction must be bromine, atomic number $35,(57+35=92)$. The fission process in this case can be represented by the equation:

$$
92^{\mathrm{U}^{235}}+\mathrm{O}^{\mathrm{n}^{1}} \longrightarrow 57^{\mathrm{La}^{147}}+{ }_{35^{\mathrm{Br}}}{ }^{87}+2 \mathrm{O}^{\mathrm{n}^{1}}
$$

It should be noted that the two fragments, although considerably lighter than the uranium nucleus, have mass numbers which are appreciably different from each other. That the uranium nucleus tends to break up in an unsymmetrical manner was first indicated by the fact that the fission fragments were observed to fall into two groups with different ionizing powers and different energies.

A more detailed study of slow neutron fission has shown that uranium-235 splits up in more than 30 different ways, for more than 60 primary products of fission have been identified. The range of mass numbers is from 72, probably an isotope of zine (atomic number 30), to 158, possibly an isotope of samarium (atomic number 62). In Figure 22 the mass numbers are plotted against the corresponding "fission yields", the fission yield being defined as the proportion (or percentage) of the total nuclear fissions yielding products of a given mass number. 


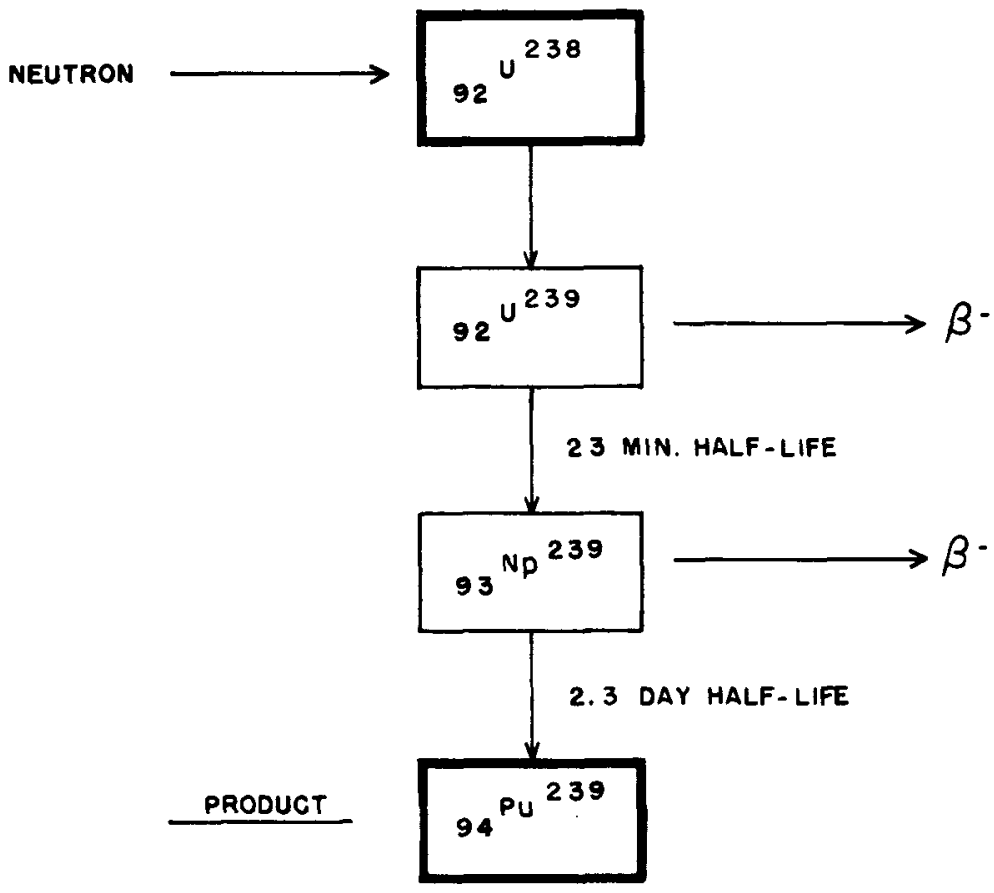

FIGURE 21

Conversion of Uranium to Plutonium

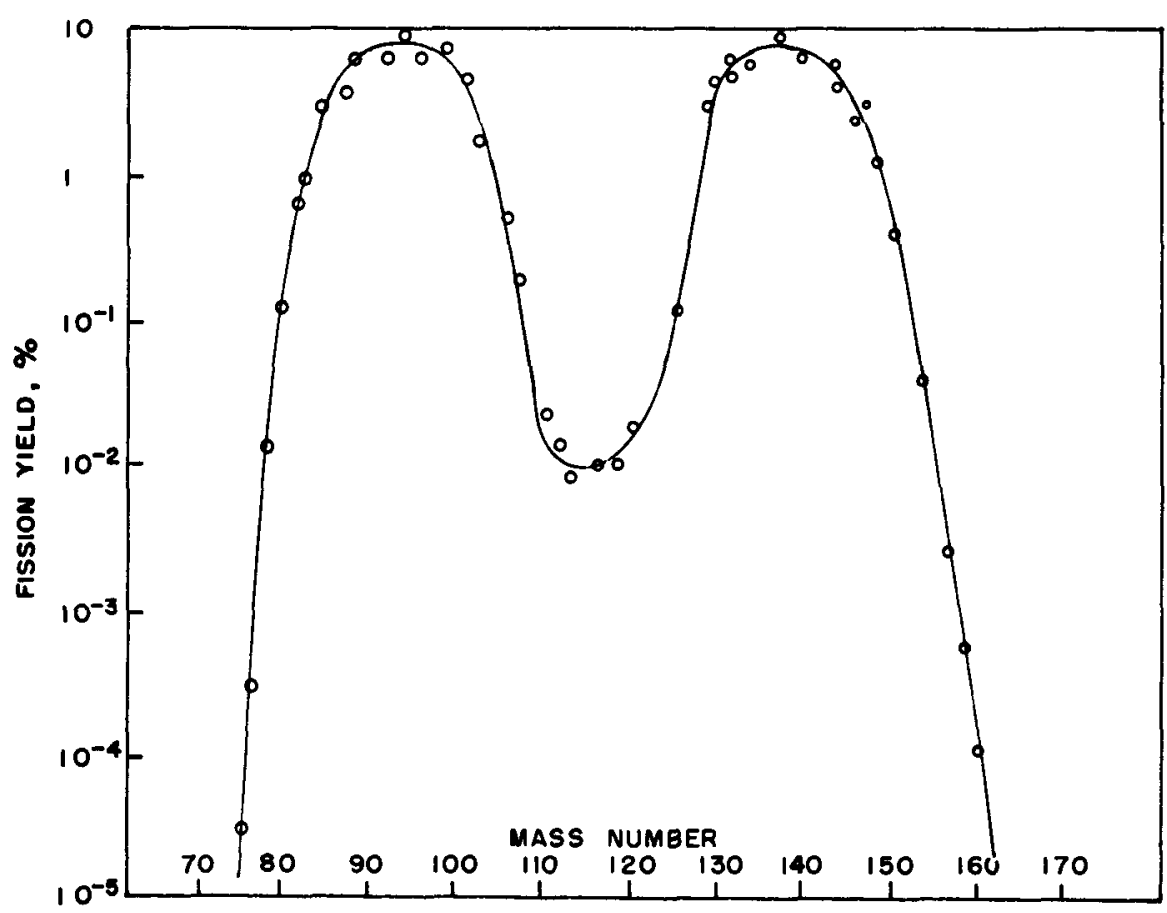

FIGURE 22

Fission Yields of Products of Various Mass Numbers 
Figure 22 shows graphically how the masses of nearly all the products fall into two broad groups: a "light" group, with mass numbers from 80 to 110 , and a "heavy" group, with mass numbers from 125 to 155 . There are some products in the intermediate range, from 110 to 125 , but altogether these represent no more than a per cent or so of the fissions. The most probable type of fission gives products with mass numbers of 95 and 139. It is apparent from these results that the thermal-neutron fission of $U^{235}$ is far from symmetrical. If the uranium nucleus had split into two equal fragments, the mass of each would be 117 or 118; only 0.01 per cent of the nuclei undergoing fission break up in this manner.

By-Products of the Reactions

The principal by-products of the above reactions arise from the fission process. The by-products of this reaction are the extremely radioactive fission fragments and a very large amount of energy per fission. One of the most significant properties of the fission products is their radioactivity. When a uranium nucleus splits up into two fragments of somewhat similar mass, the neutron-proton ratios are too high for stability. Even if a prompt neutron is expelled, the ratio of neutrons to protons will, in most cases, still be outside the stability range for the particular mass number. Consequently, all or nearly all the fission fragments are radioactive, emitting negative beta particles as well as gamma radiation. Although some decay chains are longer and some shorter, each fragment is followed, on the average, by three stages of decay before a stable species is formed.

Since there are probably some 60 different radioactive products produced in fission and each is, on the average, the parent of two others, there are about 180 radioactive species present among the fission products after a short time (after decay series start). The negative beta emission, which make up about 5 per cent of the total fission energy, give rise also to gamma rays and neutrinos. In some cases the decay results in the formation of a neutron. These "delayed neutrons" are important because 
of their effect on the control of fission rates in a nuclear reactor. The presence of the active fission products creates problems in handling and shielding against their radiations besides complicating the chemical separation of product.

Energy of Fission

The tremendous energy release, which is the other product of the fission reaction, creates great problems in heat dissipation, and consequently dictates to a considerable extent the design of the reacting units. Besides the fission fragments, other so-called prompt radiations are released at the instant of fission. The average of prompt neutrons per fission has been stated at between 2 and 3 ; these account for about 2 per cent of the total fission energy. The fission process is remarkable for the magnitude of the energy released; this is about $200 \mathrm{Mev}(200,000,000$ electron volts) for each nucleus reacting, compared with a maximum of some $20 \mathrm{Mev}$ for other nuclear reactions. In chemical reactions, the energy released is never greater than a few ev (electron volts) for each atom or molecule reacting.

Perhaps the most direct way to calculate the fission energy is to use the masses of the reacting species and of the fragments formed. The isotopic weight of $\mathrm{U}^{235}$ is 235.124 amu and the mass of a neutron on the same scale is 1.00897 , making a sum of 236.133 amu for the total mass of the interacting particles. As stated previously, the greatest yields have mass numbers of 95 and 139, which add up to 234; it may be assumed, therefore, that in this case two neutrons are freed in the fission process. The isotopic weight corresponding to the mass numbers 95 and 139 are 94. 945 and 138. 955 respectively. These, together with $2 \times 1.00897$ for the masses of the two free fission neutrons, add up to a total mass, after fission, of 235.918. The difference between this and the mass of the interacting particles before fission is converted into energy of the fission process, thus:

Mass converted into energy $=236.133-235.918=0.215 \mathrm{amu}$

$1 \mathrm{amu}$ is equivalent to $931 \mathrm{Mev}$; consequently, energy released per fission $=931 \times 0.215=198 \mathrm{Mev}$.

Unfortunately this tremendous amount of liberated energy is almost completely wasted in a production-only reactor.

UNCLASSIFIED 
Dependence of Reactions on Neutron Energies

The reactions of ${ }_{92} \mathrm{U}^{238}$ and ${ }_{92} \mathrm{U}^{235}$ are initiated most effectively by neutrons of certain definite energies. In other words, the cross sections of these nuclei are greater for neutrons having certain specific speeds or energies. In fact, the cross section (the probability of a given atom for capturing a passing neutron) of ${ }_{92} \mathrm{U}^{238}$ for capture of neutrons is greater for neutrons of two different energies. One is in the neighborhood of $25 \mathrm{ev}$ where resonance capture occurs; the other is at the thermal level, about $.025 \mathrm{ev}$, where capture also occurs. The capture by ${ }_{92} \mathrm{U}^{238}$ of neutrons of other energies is very much less probable.

Likewise, the fission of ${ }_{92} \mathrm{U}^{235}$ is initiated most effectively by neutrons of certain energies. The cross section of ${ }_{92} \mathrm{U}^{235}$ for fission is small except for the very slowest neutrons (thermal neutrons). The chance that a fission of $9{ }_{9} \mathrm{U}^{235}$ will be initiated by a neutron of other than thermal energies is relatively small (see Figure 23). The neutrons required for these reactions of $\mathrm{U}^{238}$ and $\mathrm{U}^{235}$ are themselves emitted as fast neutrons during the fission of $\mathrm{U}^{235}$. Therefore, to be effective in producing capture and fission reactions in other nuclei, these fast neutrons must be slowed down from their initial energy of about $2 \mathrm{Mev}(2,000,000 \mathrm{ev})$ to resonance (25 ev) and thermal (0.025 ev) energies.

Another instance of the chance of a reaction being dependent upon the neutron energy is provided by the fission of $\mathrm{U}^{238}$. This fission, which is of secondary importance to the general process, is initiated only by neutrons having energies greater than $1 \mathrm{Mev}$ (see Figure 24). The fast fission of $U^{238}$ makes only a small contribution, of the order of 0 to 5 per cent, to the neutron supply. The contribution is small because the fast neutrons are quickly reduced to energies less than $1 \mathrm{Mev}$ by inelastic collisions with the uranium nuclei near their point of origin. The remainder of the neutrons required are provided by the thermal fission of $\mathrm{U}^{235}$. 


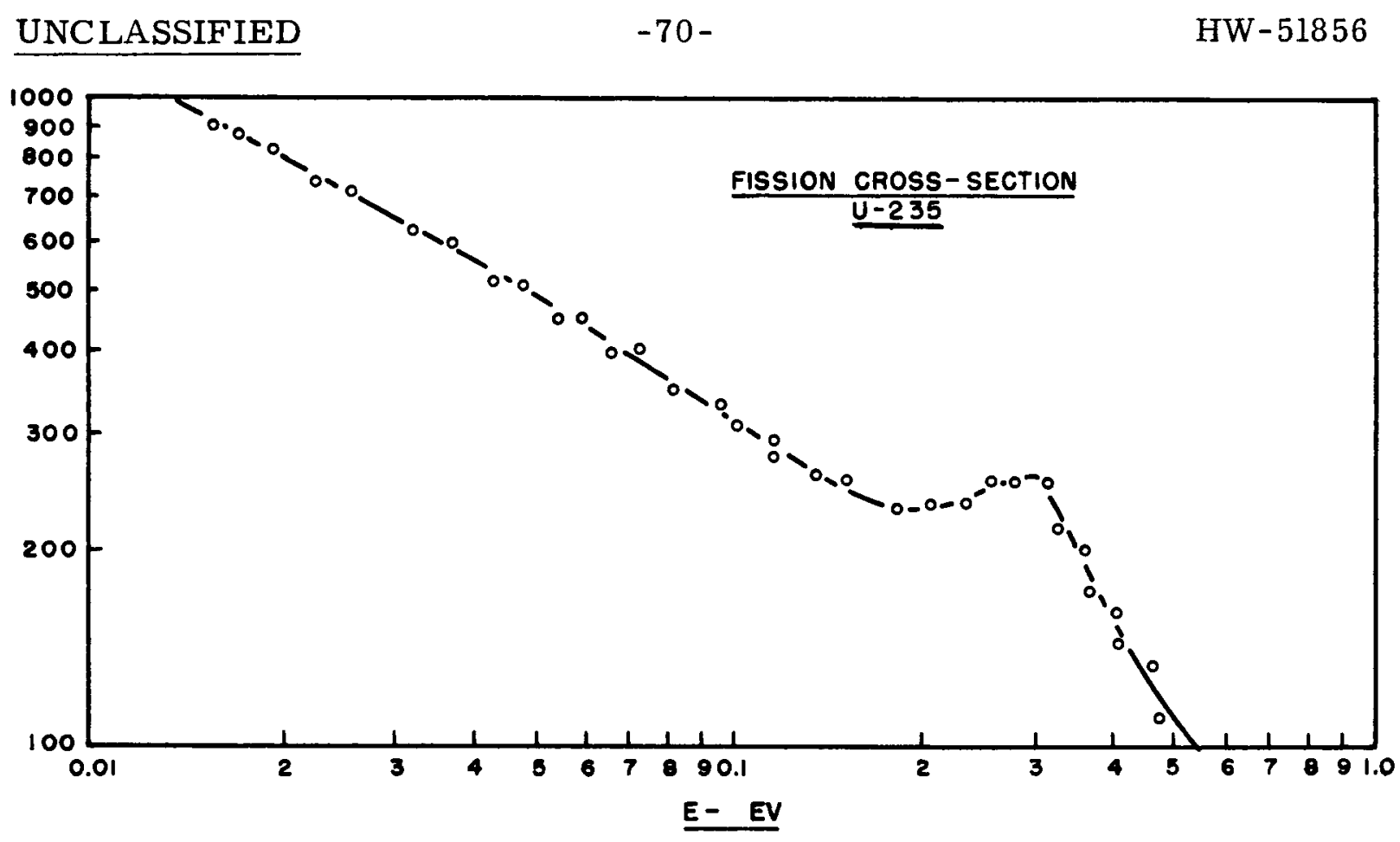

FIGURE 23

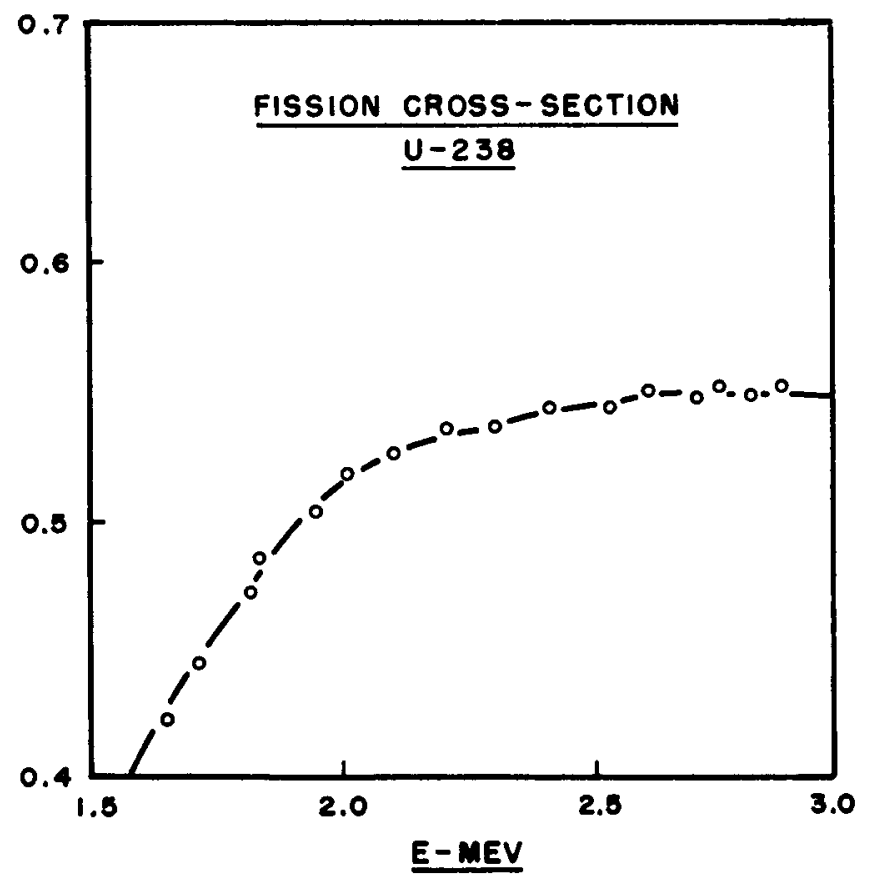

FIGURE 24 


\section{Slowing Down of Neutrons}

As discussed in the previous section on cross sections, the free "fast neutrons" released during the fission of $92 \mathrm{U}^{235}$ must be slowed down to resonance and thermal energies in order to produce capture and fission reactions in other nuclei. "Thermal" energy is the speed with which atoms of the moderator vibrate simply because of the temperature of the moderator. Thus a "thermal" neutron's speed really depends directly on the moderator temperature, or "thermal" status.

Fast neutrons are effectively slowed down in velocity by sending them into a medium which has a low atomic weight and a low neutron capture cross section. In this "moderator" medium the neutrons lose their energy by elastic collisions with the nuclei. The lower the atomic weight of this moderating medium the larger will be the fractional loss of energy by the neutrons per collision, with the result that a smaller number of collisions will reduce the neutrons to the required low velocity. Compressed helium gas or simple compounds of heavy hydrogen would make excellent moderators except for the difficulty of handling and procurement. An excellent substance to use from the standpoints of good mechanical strength, workability, cost, and availability is carbon in the form of graphite. For this reason graphite was selected as the moderating medium for the Hanford units.

From previous experiments, it was concluded that an efficient structure compatible with practical design and process requirements would be a pile of graphite blocks with alternate layers of the blocks carrying chunks of uranium. The layers of pure graphite and graphite-uranium blocks would be piled one on the other until a sufficiently large size - the calculated critical size - for a self-sustaining reaction was reached. It is from this piled structure that the name "pile" originated.

The effect of this arrangement would be that the neutrons liberated from the fission of a $\mathrm{U}^{235}$ atom would escape from the uranium chunk into the graphite, where it would be quickly slowed to the thermal level. Once in the thermal range these neutrons would be comparatively safer from 
non-fission capture by the predominant $\mathrm{U}^{238}$ atoms. In this thermal state each neutron would wander around, until, within a fraction of a second, it met a $\mathrm{U}^{235}$ atom, split it, liberated more neutrons, and forged a new link in the chain of fission. By way of summary, the inter-relation of the two fundamental pile reactions and the relation of the neutron slowing down process and by-product formation are illustrated schematically in Figure 25.

\section{CHAIN REACTION IN THE PILE}

Requirements of a Chain Reaction

The essential condition is that a self-sustaining chain reaction should be maintained; in other words, once the fission process has been initiated in a few nuclei, it should be able to continue throughout the remainder of the material without external influence. Since at least two neutrons are released in each act of fission, and these neutrons are capable of inducing the fission of other nuclei, and so on, it would appear that the requirements of a self-sustaining chain reaction can be met. However, we must take account of the fact that the neutrons produced in the fission process can take part in other (non-fission) reactions. In addition to this competition for neutrons, there is the inevitable loss of neutrons from the system by leakage.

Transmutation of uranium into plutonium on a large scale requires a continuous supply of a large number of free neutrons. These neutrons must be obtained as spares from the fission of $\mathrm{U}^{235}$. As has already been explained, the fission reaction acts as a multiplier of neutrons, since it releases several fast neutrons for the single thermal neutron captured per fission. The velocity of these fast neutrons must be reduced for capture by $\mathrm{U}^{238}$ to form plutonium or capture by $\mathrm{U}^{235}$ to cause its fission. When the conditions are so adjusted that $U^{238}$ absorbs a fraction of the moderated neutrons and still on the average leaves at least one of the new fission neutrons to continue the fission of more $\mathrm{U}^{235}$, thus completing the cycle, a "chain reaction" is said to exist. When more than one neutron 
becomes available to continue the fission in each neutron cycle or generation, the reaction will not only maintain itself but will accelerate at a rate which depends on how many spare neutrons are created each generation in addition to those needed to continue the chain reaction.

Chain Reaction

Perhaps it will be easier to understand the chain reaction if we take an atomic viewpoint. To a man's-eye view uranium is a hard white metal, almost as dense and heavy as gold - an extremely solid material. To an atom's-eye view, a mass of uranium consists of tiny, compact nuclei set in enormous systems of electrons. In each of these systems 92 electrons are stationed in shell after shell, at greater and greater distances from the nucleus. These complex atoms are distributed according to a precise, ordered system, each holding all its neighbors, and in turn being held by its neighbors, in a rigid pattern. This rigidity of pattern is what gives to the metal the property we call hardness.

The atoms are not touching; there are great distances between them. Most of the atoms that make up the uranium crystal is empty space. Into this empty space an extremely minute particle comes drifting along. It gently nudges the nucleus of an uranium atom and bounces away to wander on. It approaches anothernucleus in the same leisurely fashion, and bounces away in a new direction. The minute particle is a very slow (thermal) neutron, and the uranium atoms are $\underline{\mathrm{U}^{238}}$. The neutron then moves toward another uranium atom, an atom with the same sort of electron rings, with a nucleus of exactly the same diameter, but with 3 parts less in weight than $U^{238}$ an atom of $\underline{\mathrm{U}}^{235}$.

The slow moving neutron nudges at the $\mathrm{U}^{235}$ atom's barrier of atomic forces - and abruptly vanishes - it's inside. For an instant of time the nucleus, now an atom of uranium isotope $\mathrm{U}^{236}$, appears to be stable, then it bursts (fissions) with a violence of tremendous proportions. The nucleus ruptures and a burst of radiant energy (gamma rays) streaks away 


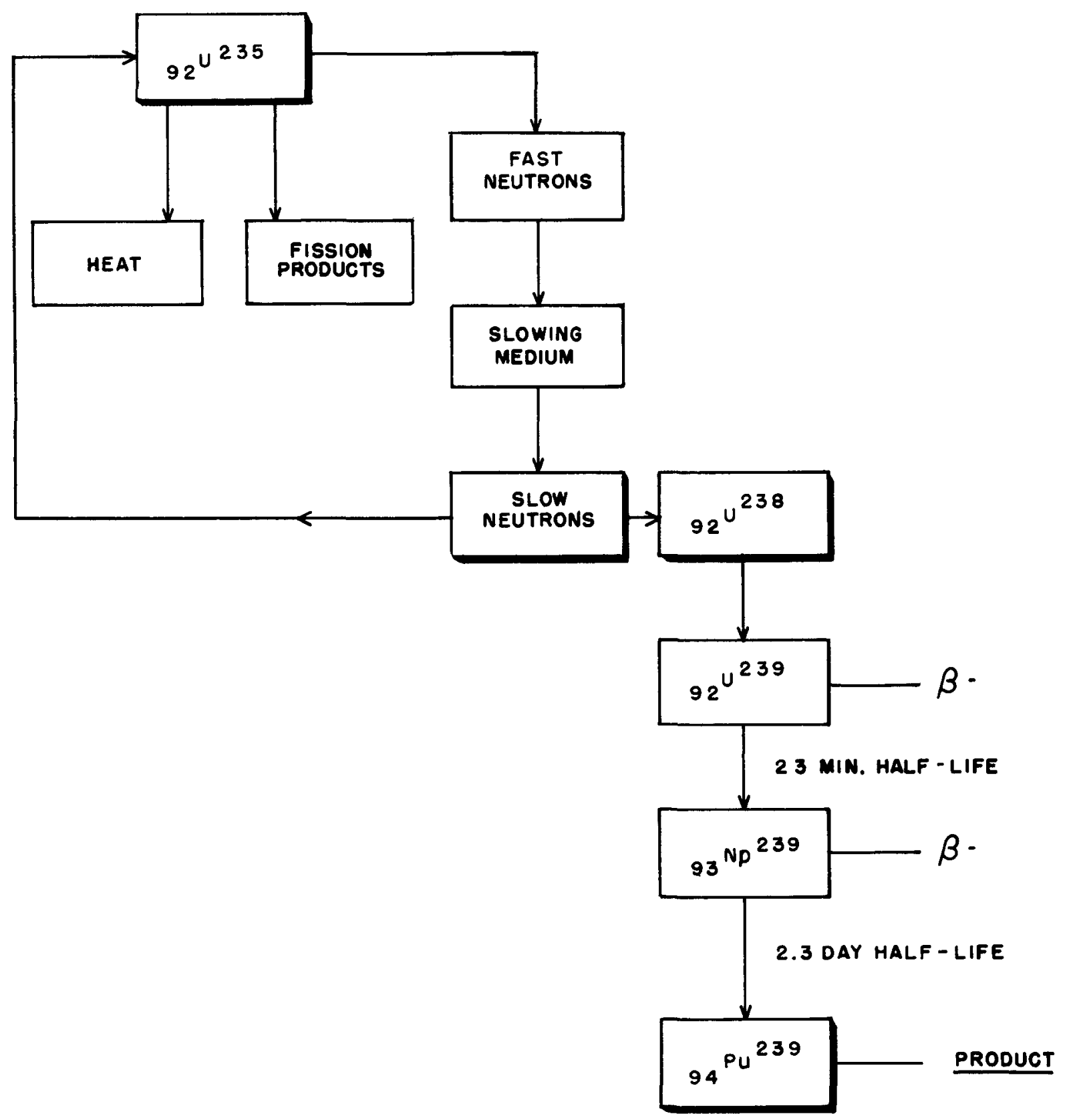

FIGURE 25

Fundamental Reactions of the Process 
at a speed of 186,000 miles a second. With the violent destruction of the atomic force barrier, the mass of neutrons and protons separate, and each fragment envelopes itself in a new and tighter sphere of atomic force. The two newly created nuclei are driving away from each other so rapidly that the distant outer electrons discover that the positively charged nucleus they were circling has suddenly vanished. Now that the electrons are no longer held together by the attraction of the nucleus and are repelled by their negative charges, the entire electron system explodes outward. The inner electrons, most powerfully bound before, and most powerfully repelled now; drive out with such violence that the first atom they hit results in a blast of hard, penetrative $X$ rays.

The two newly formed nuclei are speeding away in opposite directions. They have been smashing into other uranium atoms and finally slow down and begin to pick up stray electrons to build up new inner and outer layers of electron shells.

One of the new nuclei quivers, and its barrier wall bursts to discharge an electron, which is driven away at terrific speed. Another electron is emitted and then a neutron shoots out. It is out of the uranium in the shortest fraction of a second and collides with a nucleus of the moderator (graphite). Both the neutron and the moderator nucleus recoil and fly off in opposite directions. Collisions follow rapidly until finally the neutron has surrendered all its original kinetic energy and simply drifts along, pushing at one atom of the moderator after another, finally settling down to the same speed at which the moderator atoms are bouncing at that particular moderator temperature. It should be pointed out that this slow neutron "drift" rate is still greater than 5000 miles per hour at room temperature. Then it drifts into another uranium piece, where the nuclei are surrounded by shell after shell of electrons. The slowly moving neutron nudges a nucleus, bounces, and wanders off to bump another, until it suddenly vanishes inside a $\mathrm{U}^{235}$ nucleus and initiates another fission reaction. 


\section{Neutron Loss}

The chain reaction, as described above, is carried out in a structure called a "pile" which at Hanford consists essentially of uranium metal interspersed in graphite which acts as the moderating medium. This pile has been designed to minimize losses of neutrons and at the same time to permit thermal neutrons, slowed down by the graphite, to re-enter the uranium and initiate further fission of $\mathrm{U}^{235}$. Neutron losses in this slowing down interval can occur in the following principal ways:

1. A neutron can escape out of the pile while either fast or slow and thereby become lost to the fission process.

2. A neutron can be captured by the nuclei of some impurity present. The materials of the moderator and heat removal systems are principally responsible for this type of capture.

3. A neutron can be captured by $\mathrm{U}^{238}$ while at resonance or thermal energies. This capture, although leading to plutonium formation, results in loss of neutrons to the fission process or chain reaction.

When more than one neutron per fission becomes available to continue further fission, the number of neutrons in a pile increases exponentially; that is, it increases by a definite factor for each time unit interval. This ability of a pile to increase the number of free neutrons within it by multiplication, generation by generation, is called its "reactivity". To obtain maximum reactivity (largest multiplying ability) from a pile, the neutron losses during the slowing down process must be minimized.

\section{Critical Size}

The proportion of neutrons lost by escape from a finite reactor can be diminished by increasing the size of the system. The escape of neutrons occurs at the exterior, but absorption, leading to fission and neutron production, occurs throughout the whole of the interior of the reactor. The number of neutrons lost by escape thus depends on the 
external surface area, while the number of neutrons formed is determined by the volume. To minimize the loss of neutrons, it is necessary to decrease the ratio of area to volume; this can be done by increasing the size of the reactor.

A familiar example of this sort is found in a coal fire. Coal will burn only if it is kept hot. It produces heat in burning, but hot coal throws off heat by radiating it from all surfaces. One walnut-sized lump of coal will not readily burn; even if you heat it white hot, it will burn only briefly, for it loses heat faster than it generates it, and it quickly falls below burning temperature. But two dozen lumps of coal of the same size will burn evenly if they are in a pile. Here we have increased the volume of the coal and decreased the relative area of exposed outer surface. Since the coal generates heat throughout the pile and loses it only at the exposed outer surfaces, it will now be able to generate heat faster than it loses it; consequently it will burn.

The same concept can be used in relation to the size of the pile. As you increase the size of a cube or sphere, the surfaces increase, but the volume increases more rapidly. A cube $1 \times 1 \times 1$ foot, has a surface area of 6 square feet and a volume of one cubic foot. If we double each dimension, making the cube $2 \times 2 \times 2$, the area quadruples, becoming 24 square feet; but the volume octuples - it becomes 8 cubic feet. Thus the surface to volume ratio in the larger cube $(24 / 8)$ is only half as great as in the smaller case (6/1). This basic geometrical law is one of the principal factors in the design of atomic reactors.

The number of neutrons in a pile will be in direct proportion to the volume, whereas the number escaping from the sides will be in proportion to the surface area. Thus, the larger the pile, the smaller the proportional leakage out the sides. When proportional leakage losses become sufficiently small - this is, when the loading becomes sufficiently large - a chain reaction becomes possible, and the loading is said to be of "critical size". Since the value of " $k$ " (multiplication factor) depends on the composition of the fuel used, the type of moderator, lattice spacing, etc., it is apparent that 


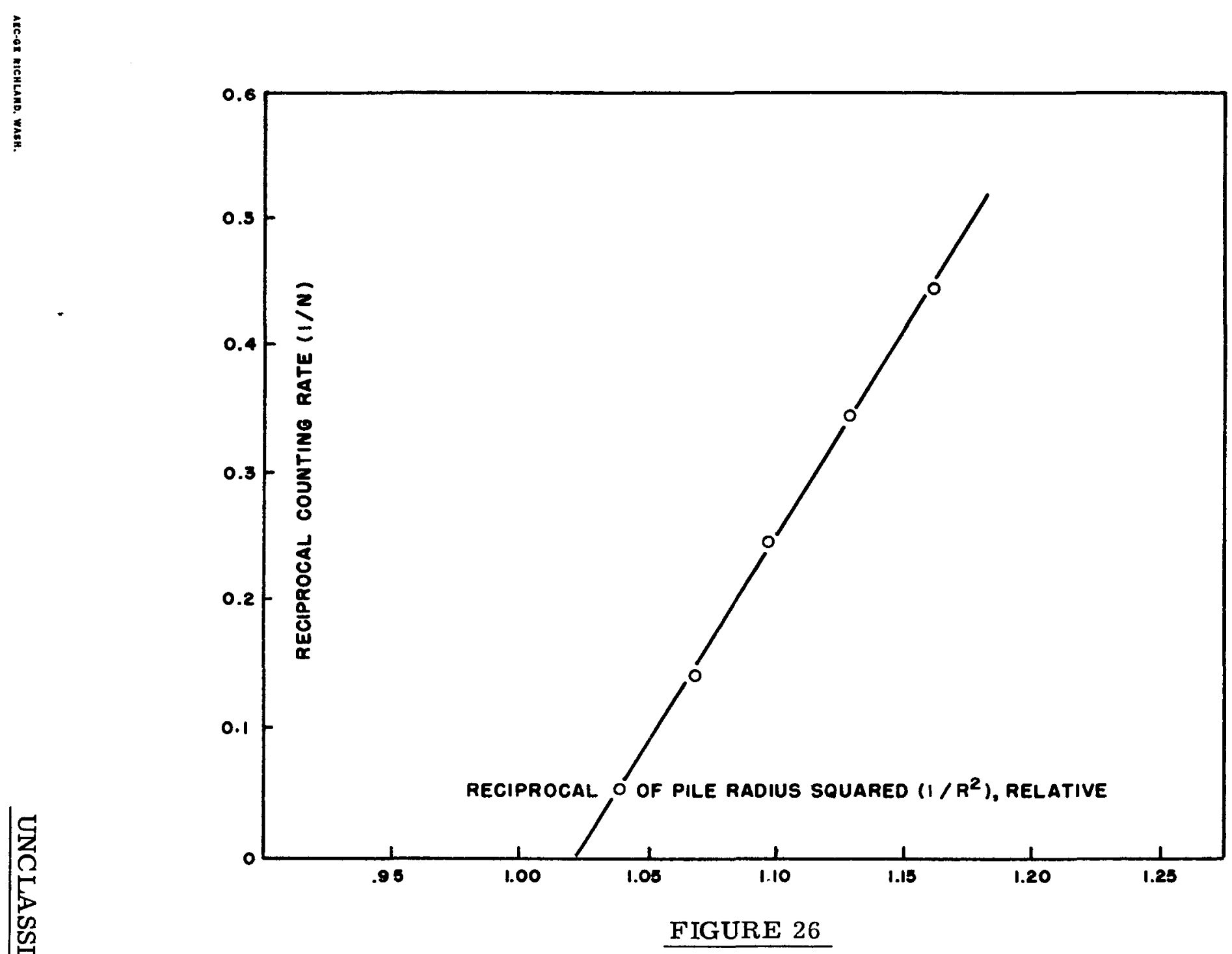




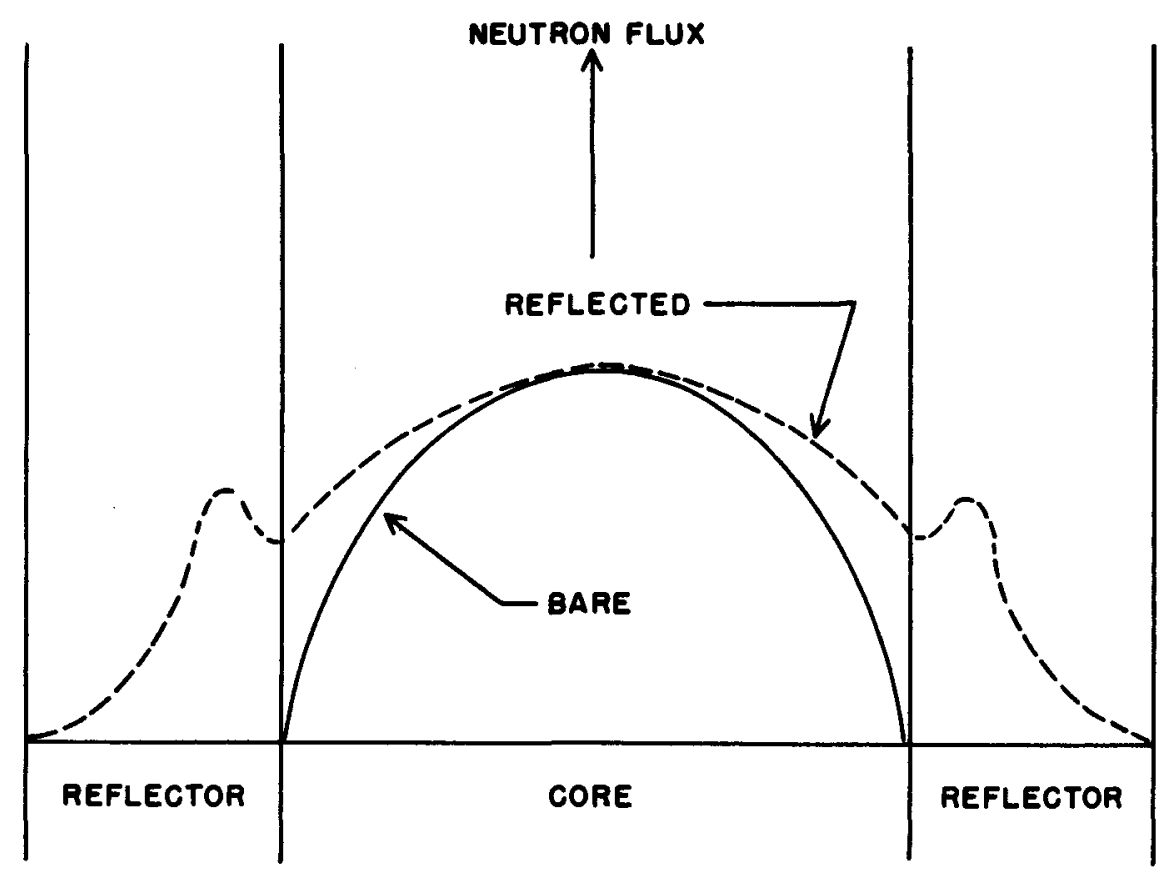

FIGURE 27

Thermal Flux Distribution in Bare and Reflected Areas 
In the design of the lattice, the spacing of the metal in the graphite and the relative amounts of metal and graphite are critical. Metal to metal. spacing which is too small leads to larger loss to $\mathrm{U}^{238}$ of neutrons of resonance energies, whereas excessively large spacing allows greater chance of capture of the neutrons by the moderator before they reach the uranium surface.

The existence of a chain reacting condition in a pile thus depends upon a rather delicate balance between neutron gain and loss in each lattice cell. Therefore, very detailed quantitative consideration of all the methods of neutron loss is necessary in designing a pile, and experiments to measure this delicate balance have to be performed to confirm theoretical calculations before the lattice design can be "frozen" for a new reactor. Most such experiments are done in a sub-critical "exponential" pile, using the same principle of background multiplication used in predicting critical size to establish the reactivity characteristics of a given lattice design.

Multiplication Factor, $\mathrm{k}$

If a chain reaction is to be maintained, the minimum condition is that for each time a nucleus captures a neutron and undergoes fission there shall be produced, on the average, at least one neutron which causes the fission of another nucleus. A chain reaction which continues indefinitely at the same rate is said to have a multiplication factor, or $k$, of one. If the chain reaction is increasing in intensity, it has a multiplication factor greater than one; if the reaction is dying off, it has a $\mathrm{k}$ less than one. Precisely, the multiplication factor, $k$, is defined as the ratio of neutrons present in one generation relative to the number present in the preceding generation.

In the complete chain reaction, every neutron loss has to be taken into account - whether it be absorbed by $\mathrm{U}^{235}, \mathrm{U}^{238}$, or moderator or whether it be lost to leakage out the sides or to the control rods. For simplification, the mathematical definition simply talks about the ratio of fission - producing neutrons per generation, considering these other losses as some "black box" obstacle which tends to reduce the multiplication factor. UNCLASSIFIED 
As with the human race, a generation survival rate of anything more than one will result in a rising population, anything less than one will mean a population decline and, if continued, eventual extinction. In other words, if every 100 neutrons (or humans) in one generation of neutrons gives birth to 101 survivors in the next generation, then the neutron (or human) population will go steadily up. Let this survival rate drop even to 99.99 and the result is inevitable annihilation. Suppose, for example, a particular generation starts with 100 neutrons; if the multiplication factor is unity, there will be 100 corresponding neutrons at the beginning of the second generation, 100 at the third, and so one. Once the chain reaction has started, the fission rate will continue at the same level indefinitely. For practical purposes, it is necessary that " $k$ " be capable of exceeding one, if a rise in power is to be attained; when the desired level has been reached, as measured by detecting chambers or temperature monitors, sufficient control rod is inserted to reduce the " $k$ " to exactly one.

Excess k

The amount by which the multiplication factor exceeds one is called the "excess multiplication factor", or excess $k$, and is usually expressed in terms of per cent of the multiplication factor. For example, a multiplication factor of 1.01 corresponds to an excess $\mathrm{k}$ of 0.01 , or 1 per cent.

The multiplication $\mathrm{k}$ is effectively the number of neutrons present at the end of a neutron generation compared to those present at the beginning of that generation. Since one neutron is required to maintain the chain reaction, the relative number of neutrons will increase by $\mathrm{k}-\mathrm{l}$ in one generation. Thus, for a given number, $\mathrm{n}$, of neutrons present initially, the rate of increase will be $n(k-1)$ per generation. Therefore, if the multiplication factor " $k$ " is greater than one, the number of neutrons will increase exponentially with time.

Suppose in a particular case that $\mathrm{k}$ is 1.005 , so that $\mathrm{k}$ ex (excess $\mathrm{k}$ ) is 0.005. Taking the average neutron lifetime in a thermal reactor to have the reasonable value of 0.001 seconds (and neglecting for the 
moment the effect of delayed neutrons which will be discussed later), it follows that after a lapse of one second the number of neutrons would have increased roughly 150 fold. An increase in the number of neutrons will mean an increase in the fission rate and a consequent increase in the power output. When the desired output is attained, the multiplication factor should be reduced exactly to unity by the insertion of a nonfissionable neutron absorber, such as a control rod. The number of neutrons present in the pile, the fission rate, and the power level will then remain constant.

If the multiplication factor is less than unity, the maintenance of a self-sustaining chain reaction becomes impossible; the rate of change in the number of neutrons is now negative, so the neutron concentration decreases continuously in an exponential manner. As long as the multiplication factor is less than one, by no matter how small an amount, the number of neutrons must inevitably decrease with time until a background level is reached, and the maintenance of a self-sustaining reaction is not possible.

\section{k Infinity}

Imagine for a moment that all space is filled to infinity with block on block of reactor lattice cells. Then, obviously there won't be any place for a neutron to escape to that isn't still in the scene of action. This concept, for which there is no leakage of neutrons (represented by the symbol $\mathrm{k}_{\infty}$ ), is called the "infinite multiplication factor". A chain reaction is one which sustains itself because the proper balance between fissionable and non-fissionable atoms replaces to the process the same number of neutrons that are lost or absorbed. If this proper balance can be achieved, and no other neutrons escape from the reactor, we can say that the reactor has an infinite pile reproduction factor or $\mathrm{k}_{\infty}$ of 1.000 .

If we measure the critical size of a given lattice design, we can calculate from the critical dimensions what the $\mathrm{k}$ excess would have been stretched to infinity and thus had suffered no neutron leakage. We then have determined a physical constant describing this particular lattice cell 
design, regardless of the size or shape of the pile in which it's located. This is somewhat like saying that a Winesap limb will produce Winesap apples even if grafted to a Delicious tree. This concept of $\mathrm{k}_{\infty}$ - or what would a particular lattice design act like if it were infinite in extent - is thus useful for theoretical calculations.

k Effective

We have stated that the $\mathrm{k}$ multiplication factor is the number of neutrons present at the end of a neutron generation relative to each neutron present at the beginning of that generation. In a practical case, however, some of these neutrons are bound to escape from the surface of the reactor. Thus, the chain reaction has to strike a balance between neutron-producing fissions and neutrons lost to product formation plus leakage (or escape). If we can exactly achieve this three-way balance chain reaction, the reactor has an "effective reproduction factor", or $\mathrm{k}_{\text {eff }}$, of 1.000000.

What would happen if we designed a pile to operate with a $\mathrm{k}_{\text {eff }}$ greater than one. Suppose we had a reactor using only fissionable atoms and so large that it had a $\mathrm{k}_{\mathrm{eff}}$ of 2 . The first generation of neutrons would have 1 , the second 2, the third 4, the fourth generation 8 , etc., until the twentieth generation had over a million. It is quite obvious that we must be sure that the $\mathrm{k}_{\text {eff }}$ of the pile never gets much above 1.0000 if we wish to control it. $\underline{\text { Summary of } \mathrm{k} \text { Terms }}$

Four $\mathrm{k}$ terms have been mentioned which are likely to cause confusion. The initial term, " $k$ ", was introduced simply to get across the concept of the multiplication factor. The term "k effective" is the one actually used to describe the generation rate of neutrons in a pile. The term "k excess" is used in the same manner and is equal to "k effective" minus one; the term "reactivity" is usually considered in terms of excess $\mathrm{k}$ or an equivalent scale such as inhours or dollars. The term "k infinity" is somewhat hypothetical because it is inferred from critical size measurements rather than being possible of direct measurement. These terms are used in the following paragraph to illustrate their distinctions.

UNCLASSIFIED 
As stated previously, the number of neutrons in a pile will be in direct proportion to the volume, whereas the number escaping from the sides will be in proportion to the surface area. Thus, the larger the pile, the smaller the proportional leakage out the sides. We can now see that if we build a pile with a lattice design having a given " $k$ infinity" greater than 1.000000, there will be some finite size at which the leakage out the sides will just exactly match the extra neutrons produced inside. When the pile is built to this size at which it barely supports a chain reaction (has a $\mathrm{k}$ effective of 1.000 ) it is said to be critical size. By measuring the pile with a yardstick at the size at which it barely goes critical, we can calculate the "k infinity" or basic reactivity properties of this particular pile's lattice design. The first requirement in building a pile is to use a lattice design which is inherently reactive enough to support a chain reaction in the size of pile we wish to build, but not so overly reactive that it causes unnecessary potential control problems.

The critical condition is that the effective multiplication factor ( $\mathrm{eff}_{\mathrm{ef}}$ ) shall be exactly unity. The chain reaction will then be maintained at a constant rate of fission and power level, referred to as the "steady state" of the reactor. A given reactor can have an indefinite number of such states corresponding to different fission rates and power levels. If the $\mathrm{k}_{\text {eff }}$ of a reactor exceeds unity, the system has some "excess $\mathrm{k}^{\prime}$, or is said to be supercritical. In such a reactor the rate of fission and hence the neutron population and power level increase steadily. When the $\mathrm{k}$ eff factor is less than unity, i.e., in a subcritical reactor, the neutron density (or flux) and power level decreases steadily.

Neutron Balance in a Chain Reaction

The value of the multiplication factor in a graphite-uranium pile depends on the relative extents to which the neutrons take part in four main processes. This detailed breakdown of neutron reactions in the chain reaction cycle is referred to as the "neutron balance". 
The four main processes are: (a) complete loss or escape of neutrons from the pile, referred to as leakage; (b) non-fission capture by $\mathrm{U}^{235}$ and $\mathrm{U}^{238}$, designated as resonance capture since it occurs mainly at resonance energies; (c) non-fission capture called parasitic capture by the graphite, structural materials, coolant, fission products, and impurities in the uranium and in the graphite; and (d) fission capture of slow neutrons by $\mathrm{U}^{235}$, or of fast neutrons by both $\mathrm{U}^{235}$ and $\mathrm{U}^{238}$. In each of these four processes neutrons are removed from the system, but in the fourth process (the fission reaction) other neutrons are generated to replace them. Therefore, if the number of neutrons produced in the fission process is just equal to (or exceeds) the total number lost by escape and by fission and non-fission capture, the multiplication factor will equal (or exceed) unity, and a chain reaction should be possible.

An illustration of the type of neutron balance that might exist in a system for which a multiplication factor is exactly unity is shown in Figure 28. It is assumed that fission results only from the capture of slow neutrons, and that exactly two and one-half neutrons are produced, on the average, in each fission process. It is seen from this hypothetical case, which might apply to a reactor in which natural uranium is the fuel, that a total of 100 neutrons is either absorbed or leaks out, and that 100 neutrons are produced by fission. This condition represents a stationary, self-sustaining chain reaction. If a total of more than 60 neutrons had leaked out and been captured by non-fission processes, there would have been less than 40 neutrons available to cause fission of $\mathrm{U}^{235}$, and consequently less than 100 fast fission neutrons would have been produced. Such a chain would decrease the number of neutrons present in the system and the reaction would cease in a short time. On the other hand, if the leakage and non-fission capture accounted for less than 60 neutrons, there would have been more than 40 fissions, and more than 100 fast fission neutrons would result. The increase of the number of neutrons present in the system would result in an increase in the fission rate and a consequent rise in the power output. 
100 fast (fission) neutrons.

$\downarrow$

85 slow neutrons arrive af thermal energy without loss or capture.

5 neutrons lost by leakage during moderation.

$\rightarrow 10$ neutrons undergo resonance absorption in $U^{238}$ to give plutonium.

1

80 slow neutrons available for absorption.

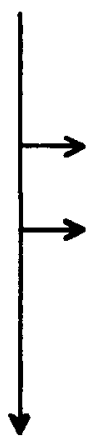

5 lost by leakage as slow neutrons.

40 slow neutrons absorbed by $U^{235}$ to cause fission.

$\downarrow$

100 fast (fission) neutrons.

\section{FIGURE 28}

Life Cycle of a Single Generation of Neutrons

(From "Elements of Nuclear Reactor Theory, "Glasstone \& Edlund) 


\section{PROPERTIES OF A CHAIN-REACTING PILE}

\section{General Description}

The Hanford reactors consist of large structures made up of graphite blocks arranged in the form of rectangular prisms. The graphite prisms are pierced with parallel holes in the form of cylindrical channels, arranged in a square pattern approximately 8 inches on a side. Each channel is lined with an aluminum tube, in which are centered the cylindrical shaped pieces of uranium metal. Cooling water is forced at high velocity through an annular space between the metal pieces and the aluminum tube. To avoid chemical action between the water and the uranium the metal is jacketed with aluminum. A graphite shell surrounds the metal-bearing inner volume. This shell of dead graphite acts as a "reflector" to return escaping neutrons to the active, inner volume of the reactor.

The graphite stack is surrounded on all sides by shielding to absorb the intense gamma and neutron radiations which are emitted during operation. Perpendicular to the production channels are holes for control rods and test and monitoring facilities. Two purposes must be met by the control system. "Shim" control is used to adjust the $\mathrm{k}$ effective of the pile to the desired amount during operation, whereas "safety" control is necessary to shut the pile down quickly in event of an emergency and to ensure that it remains subcritical when operation is not desired.

Control Rods

The adjustment of the multiplication of neutrons in a Hanford reactor is achieved by the insertion and withdrawal of control rods. The control rods contain materials such as boron or cadmium which have large cross sections for slow neutrons. They are movable so that their position in the pile can be adjusted to absorb just the desired amount of the excess multiplication factor. In order to shut down the reactor, the control rods are inserted to an extent that permits them to absorb additional neutrons. The reactor now loses neutrons faster than they are formed by fission, the effective multiplication factor sinks below unity, and the chain reaction dies out. 
The fact that control rods can be used means that the pile loading is larger than critical size - otherwise no excess reactivity would be available for control. The potential excess made available for control flexibility depends upon requirements. A low level research reactor may need only a fraction of one per cent $\mathrm{k}$ to allow it to climb to operating level, whereas a power or production reactor might need considerable more potential excess to compensate various reactivity coefficient effects. Furthermore, control rods can be used to adjust flux distribution since it is possible to compensate a given amount of $\mathrm{k}$ excess with any number of rod configurations. The amount to be invested in control flexibility is generally restricted by two considerations:

1. Neutrons absorbed in control rods are then not available for plutonium formation, and

2. A large potential excess normally compensated by control rods tends to increase the chances for startup accidents.

The slow control type of system used for normal operation generally depends on a mechanical or electrical drive, whereas the fast control type of system for emergency shutdown may depend upon a cocked spring, a cocked hydraulic system, or gravity itself for its fast action.

Effect of Operation on Multiplication Factor

The effect of different variables on the reactivity of a reactor is expressed in terms of their effect on the multiplication factor. An important variable which affects the multiplication factor is the pile temperature. The change in $\mathrm{k}$ per degree temperature change is called the temperature coefficient. The temperature coefficient arises from a complex combination of many effects. The principal ones are believed to be: (1) change of leakage rate out of the pile, (2) distortion of the relative energy distribution of slow neutrons, and (3) variation of absorption cross sections with temperature.

Several of the fission products from the chain reaction are known to have large neutron absorption cross sections. The accumulation of these products during operation consumes excess multiplication factor originally

UNCLASSIFIED 
designed into the pile. These products in effect "poison" the reactor. A compensating effect is the transmutation to other elements of highly absorbent elements which exist as impurities in the pile materials. For example, the boron content of graphite is reduced by neutron capture in the reaction $\mathrm{B}^{10}$ $(\mathrm{n}, \alpha) \mathrm{Li}^{7}$ by which less objectional lithium is formed. Plutonium formation also increases the multiplication factor because the plutonium itself undergoes fission and, therefore, causes the release of neutrons in the same manner as $\mathrm{U}^{235}$; of course burnout of $\mathrm{U}^{235}$ produces an opposing effect. Neutron Distribution

The neutron distribution in a uniformly loaded cubical pile, when not disturbed by strong neutron absorbers such as control rods, is a simple function of position in the pile. The neutron flux (or density) in this case varies along each axis (central line) according to a sine function as illustrated in Figure 29. The maximum neutron density and consequently the maximum rate of heat generation and product formation exists at the center of such a pile. The active metal-bearing portion of the pile is surrounded by layers of graphite to form a reflector for the excess neutrons which tend to drift outward from the center. The presence of this reflector modifies, somewhat, the neutron density at the edge of the metal-bearing portion, increasing the "effective" size of the pile as indicated by the actual flux distribution. Insertion of a control rod distorts the neutron distribution; the type of distortion which the control rod produces is shown in Figure 30.

Although the control rod absorbs neutrons from its immediate neighborhood, the effect of this local absorption is partially smeared out over all parts of the reactor somewhat like air pressure tends to equalize throughout a tire even though it's losing air at only one point.

The effect of a change in control rod position on the reactivity of the reactor depends on the position of the control rod in the reactor. If the tip of the control rod is in a region of low neutron flux a larger movement of the rod is necessary to effect a given change in $k$ than when the rod tip is in a 
region of higher neutron flux. Because of the variation of neutron density with location in the reactor, the reactivity effect per unit length of control rod varies along its length, and mechanically equivalent rods in different parts of the reactor are not necessarily equivalent in control strength.

Control of Activity

"Control" of activity implies that one can tell that the process is under control. Since our chain reaction is a neutron cycle, a method must be used whereby we can "see" neutrons or the side effects which they produce. Instruments are used, therefore, to measure neutron activity rates or to measure some by-product of the chain reaction such as fission product gamma radiation or rate of fission heating of the coolant. The operator uses these indications to determine whether, when, and to what extent to adjust control rod positions.

When the multiplication factor of a pile is made exactly equal to unity, by accurately positioning the control rod, the rate of heat generation or power level will remain constant - an "equilibrium" exists, as thermal neutrons are absorbed at the same rate as they are being formed. When the regulating rod is located so as to create such an equilibrium condition, it is said to be at the "critical position". Change in power level can be compared with change of direction in an automobile, the extent of the regulating rod movement corresponding to the amount the front wheels of the automobile are turned from the straight-ahead position. The extent of the change in power level of the pile, or in the displacement of the automobile, is proportional to the time the regulating rod, or the wheels, are removed from the standard position. To change the power level it is necessary to change the number of neutrons in the pile. The number of neutrons is altered by temporarily changing the multiplication factor, either by withdrawing or further inserting the regulating rod to raise or lower the power level as desired. When the desired power level (neutron density) is attained, the multiplication factor is again brought back to unity by moving the regulating rod to the critical position. The reactor will then continue to operate at the new power level. The automobile steering analogy again applies; the car will go in a straight line again UNCLASSIFIED 


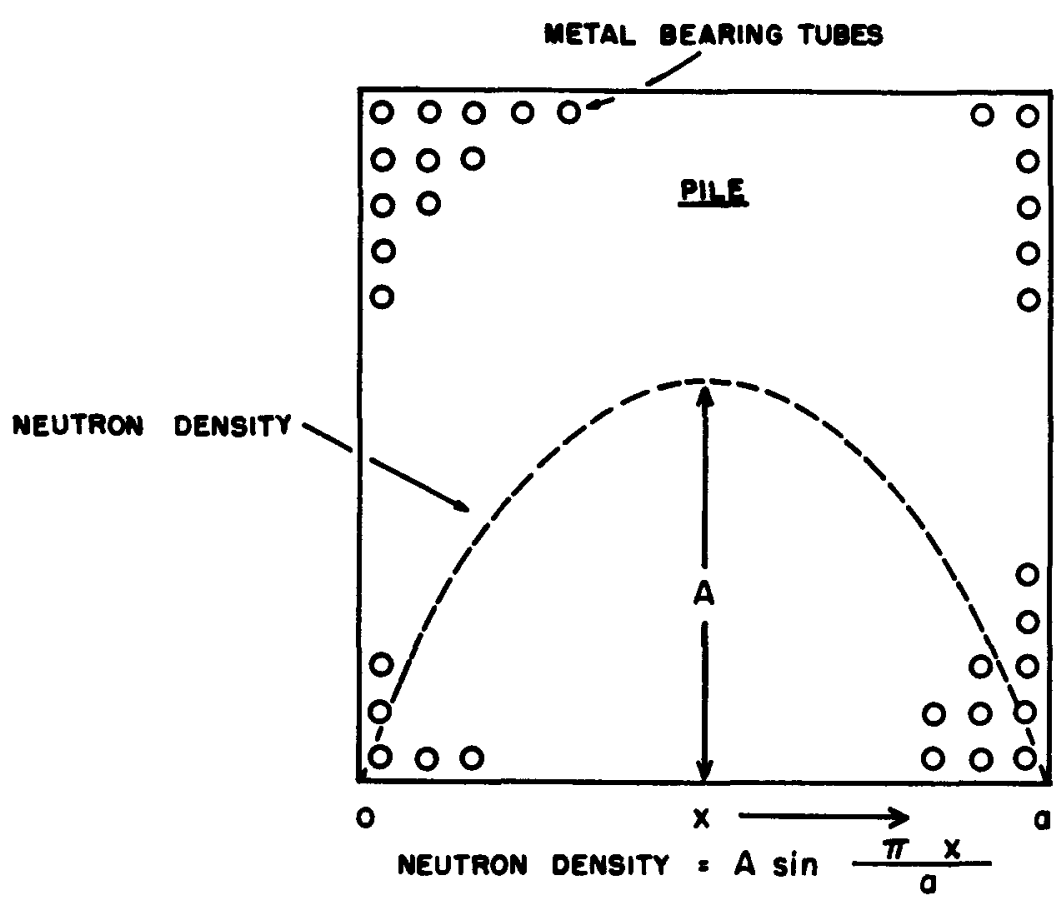

FIGURE 29

Variation of Neutron Density Along an Axis of Cubicle Pile

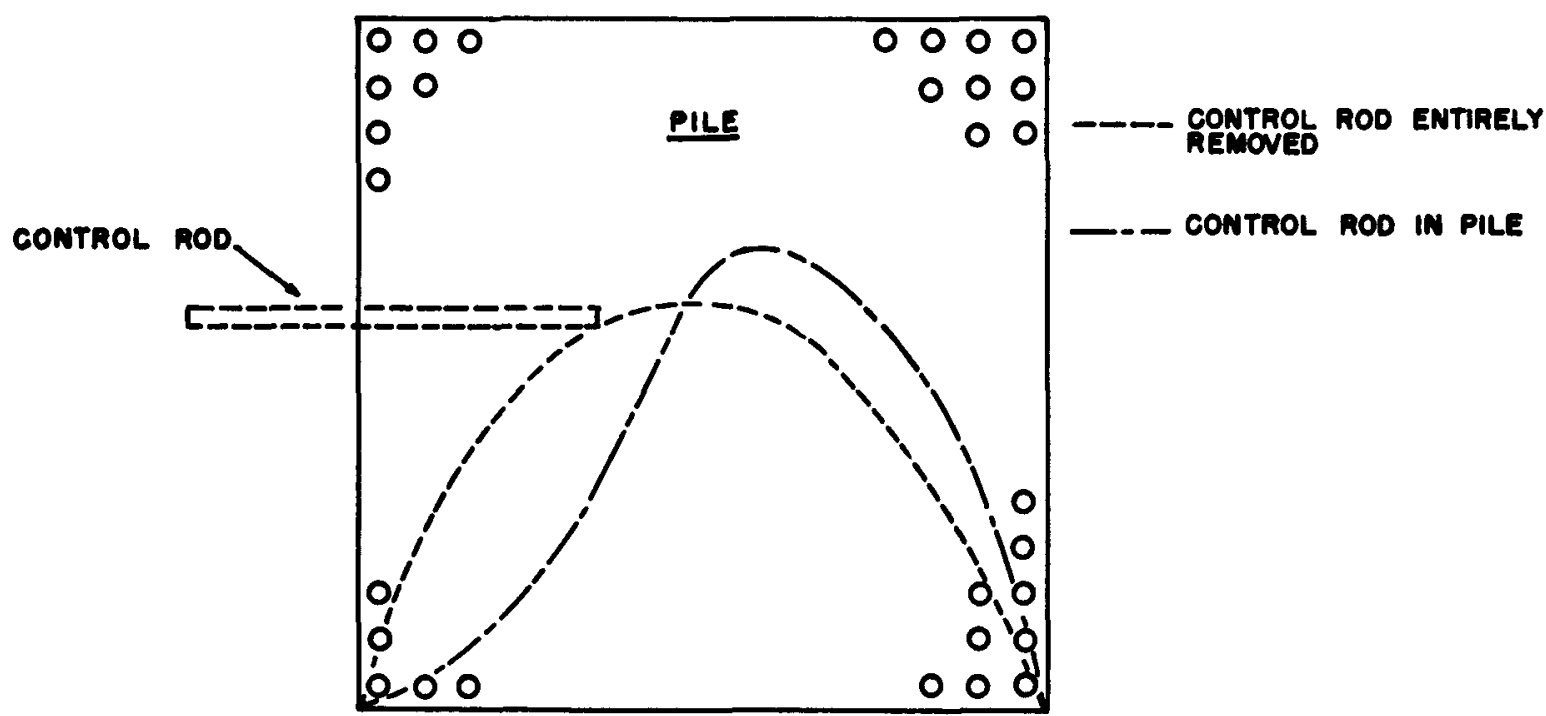

FIGURE 30

Neutron Density Along Control Rod Channel 
with the steering wheel returned to its original position; the car will be displaced sideways, however, the amount depending on the time during which the steering wheel was turned. The magnitude of the power level change is proportional to the time the neutron density is allowed to multiply before the multiplication factor is brought back to unity. Effect of Delayed Neutrons

When an atom of uranium fissions, two or more fast neutrons are ejected at speeds of thousands of miles per seocnd. They have to travel only millionths of an inch before reaching other atoms and only a few centimeters before they have been slowed down and have reached another uranium piece in the pile. The cycle from neutron caused fission through the expulsion of new neutrons, slowing of these neutrons, and the reaction of the new neutrons with other uranium atoms, all occurs in a very small fraction of a second.

The average neutron lifetime in a thermal reactor is on the order of a thousandth of a second $(0.001 \mathrm{sec})$ from the time of ejection to absorption. This means that a thousand generations of neutrons could potentially be generated in a reactor within one second. Such a rapid rate of increase in the power output would make the reactor go out of control in a few seconds. This estimate is based on the assumption that all of the fission neutrons are released at the instant of fission.

Fortunately, this is not the case; about 0.75 per cent of the total number of neutrons released in the fission reaction are "delayed neutrons" which appear an appreciable period of time after the actual fission reaction in fact, there are several delay groups ranging from 0.6 to 80 seconds and averaging about 10 seconds. This delayed neutron phenomenon, of course, affects the calculation of the rate of neutron increase (or decrease). By saving out only 1 per cent of the fission neutrons so that they are ejected from the fission particles a few seconds after the actual fission occurs, the average time of a neutron generation is changed from one-thousandth of a second to 
one-tenth of a second, and the fission reaction appears reasonable from the standpoint of human control; now an increase of 1 per cent per cycle would result in a multiplication of around 10 per cent in one second. Even an increase of 10 per cent per seond would be very fast because it would mean more than a factor of 100 per minute. Therefore, the operating excess $\mathrm{k}$ is kept at a small fraction of one per cent $\mathrm{k}$ in order to ensure safe control.

Since only $3 / 4$ of one per cent of the neutrons are delayed, the chain reaction cycle time will fast tend to approach the average neutron lifetime of $1 / 1000$ second if $\mathrm{k}$ effective increases beyond 1.0075. A k excess of 0075 is therefore called "primpt critical" and would permit an extremely hazardous rate of rise if such a reactivity excess were allowed to continue.

When a reactor is shut down with control rods, a large number of neutrons will be emitted by delayed emission and thereby cause the power to drop off over a period of several minutes rather than instananeously. Factor of e - Period

The activity, or level, of a supercritical reactor (one having a $\mathrm{k}$ excess) multiplies itself a specific amount in each minute, provided that no side reactivity effects such as control rod movement or temperature change occur simultaneously. An equivalent statement is that the level of a supercritical reactor doubles itself during a specific number of seconds or minutes provided no side reactivity effects occur simultaneously.

In actual practice, it is mathematically more convenient to use a "magic number" called e, which is equal to 2.71828 , rather than a factor of two as the multiplying factor. We then define the time taken for the level to multiply itself by 2.71828 , as the period. For a given excess k, there will be a given period; the greater the $\mathrm{k}$ excess, the faster will be the rate of rise and, therefore, the shorter will be the period. 
The mathematical series whose sum is this magic number $(2.71828)$ can be seen to describe the result of one thing acting on another, a second on a third, a third on a fourth, etc.

$$
\mathrm{e}=1+\frac{1}{1}+\frac{1}{1 \times 2}+\frac{1}{1 \times 2 \times 3}+\frac{1}{1 \times 2 \times 3 \times 4}+\frac{1}{1 \times 2 \times 3 \times 4 \times 5}+\text { etc. }
$$

The later terms fast approach zero, and in the limit of using all the terms you wish the term e equals 2.71828----. This term comes out in the solution of all problems of this type which resemble compound interest; therefore, it saves additional calculational steps in many cases to define a pile period as the time taken for the neutron level to change by this factor of 2.71828 .

Periods of the order of several seconds to several minutes are within a reasonable range for human and instrument response.

Reactivity and Period

If only those neutrons which arise instantaneously during the fission process were present in a pile, the growth of activity, as expressed by the period, would be a relatively simple function of the length of a neutron generation and the multiplication per generation, or multiplication factor. The presence of delayed neutrons, even though only to the extent of less than 1 per cent of the total emitted per fission, complicates the otherwise simple relation between $\mathrm{k}$ excess and period. The activity of these delayed neutrons decays in the same manner as the activity of more common radioactive materials; thus they appear some seconds after the creation of the fission products which emit them. As a result, the delayed neutrons contribute relatively less when the activity is rising rapidly than they do when the power level is nearly stationary. On the other hand, when the power level is falling the delayed contribution is relatively greater. Another way of looking at it is to say that if the pile is on a very fast rising period, it doesn't have time to wait for the delayed neutrons - high level is reached before all of the neutrons created at the low level have had a chance to be 
released, whereas delayed neutrons produced during equilibrium operation tend to make the shutting down process more sluggish. Furthermore, a minute or more is required after a change in rod position before the rate of neutron change is representative of a true period - a sudden jump or a sudden loss in "prompt" neutron population occurs first.

Although the relation between period and $\mathrm{k}$ excess is somewhat complicated, it is real; therefore pile periods are a valuable source of fundamental reactivity data.

Inhour

A unit of excess reactivity ( $\mathrm{k}$ excess) may be defined for operating convenience in terms of the rate of change in the pile neutron level with time. A common relation between $\mathrm{k}$ excess and pile period is the "inhour". One inhour is defined as the excess reactivity necessary to cause the neutron level to increase by a factor of e(2.71828) in one hour. Two inhours of excess would cause an increase in neutron level by a factor of e in 30 minutes (twice as fast), 3 inhours in 20 minutes (three times as fast); etc. As the period becomes shorter, the different delay times of the various delayed neutron groups cause the simple time relation to break down, requiring an equation of several terms to give an exact relation between shorter periods and greater amounts of excess activity.

The constants in the inhour equation were obtained by consideration of the half-lives and relative activities of the several delayed neutron emitters and the average length of the neutron generation in the pile. They of course were also chosen to give a pile with a period of one hour a reactivity of one inhour. A direct relationship exists between inhours and units of $\mathrm{k}$ excess; it just may be more convenient in pile operation to talk in terms of periods and inhours rather than periods and excess $\mathrm{k}$. 


\section{Semi-Log Paper}

We are used to multiplying numbers directly to find their product; and this is what needs to be done to determine activity level when a pile is on a free period, such as $1.01 \times 1.01 \times 1.01 \times 1.01$, etc. But there is a short way of indicating the multiplying process if a number is going to be multiplied by itself several times. For example, $2 \times 2 \times 2 \times 2=16$ can also be written $2^{4}=16$. Thus $10^{1}=10,10^{2}=100,10^{3}=1000$, etc. (See Section A, Item - Powers of Numbers).

Since a pile with an excess $\mathrm{k}$ is multiplying its power level by itself over and over again, it becomes quite useful to show this multiplying process as an addition of powers. By printing graph paper such that evenly spaced lines prepresent a "power" rather than a final product, a compound interest rate type of increase with time takes up equal spaces when plotted on a scale of powers, whereas the true rise becomes greater each succeeding increment of time. For example, the distance between the 3 and the 6 on a logarithmic scale is the same as that between the 6 and the 12 , the 12 and the 24 , etc. In other words, the larger the quantity, the more squashed down is the scale. The advantage of this type plot is that the rate of multiplication shows up as a straight line and can thus be quickly judged. An illustrative plot of proportional counter data during a reactor startup is shown on semilog paper in Figure 31

Radiations

Operation of a pile is accompanied by the emission of large amounts of radiation in the form of gamma rays, neutrons, fission fragments, and beta particles. The energy distribution among the various types of radiation and the location of their energy absorption are given in the following table: 


\section{DISTRIBUTION OF ENERGY IN A HANFORD REACTOR}

\begin{tabular}{|c|c|c|c|c|}
\hline & Mev/Fission & $\begin{array}{l}\text { Absorption } \\
\text { in U } \\
\end{array}$ & $\begin{array}{c}\text { Absorption } \\
\text { in Carbon }\end{array}$ & $\begin{array}{l}\text { Absorption } \\
\text { in Shields } \\
\end{array}$ \\
\hline $\begin{array}{l}\text { Kinetic Energy of } \\
\text { Fission Fragments }\end{array}$ & 168 & All & 0 & 0 \\
\hline $\begin{array}{l}\text { Kinetic Energy of } \\
\text { Neutrons }\end{array}$ & 5 & & Nearly All & $\ll 1 \%$ \\
\hline $\begin{array}{l}\text { Gamma Rays from } \\
\text { Fission Products }\end{array}$ & 5 & $>50 \%$ & $<50 \%$ & $\ll 1 \%$ \\
\hline $\begin{array}{l}\text { Beta Emission from } \\
\text { Fission Products }\end{array}$ & 7 & All & 0 & 0 \\
\hline $\begin{array}{l}\text { Gamma Rays from } \\
\text { Activated Components }\end{array}$ & 6 & $>50 \%$ & $<50 \%$ & $<5 \%$ \\
\hline
\end{tabular}

The energy of these radiations is converted into heat in the absorbing materials. For this reason the cooling system must be designed to remove heat from all parts of the reactor, possibly including the outer radiation shields.

The activity associated with operation at a $1 \mathrm{kw}$ power level is estimated to be equivalent in ionizing power to 12 pounds of radium; the present world supply of radium is less than 5 pounds. The radium equivalent of the ionization of a single large production or power reactor at operating level may be on the order of hundreds of tons of radium.

Gamma rays and neutrons are extremely penetrating and, therefore, leak out of the active graphite portion of the pile. Both of these radiations are harmful to human tissue, so safeguards against irradiation of personnel are necessary. After a reactor is shut down the neutron emission decays to a negligible amount in the order of minutes but the emission of gamma rays from the active fission products continues, and is still on the order of onetenth of its original shutdown value after several days. 


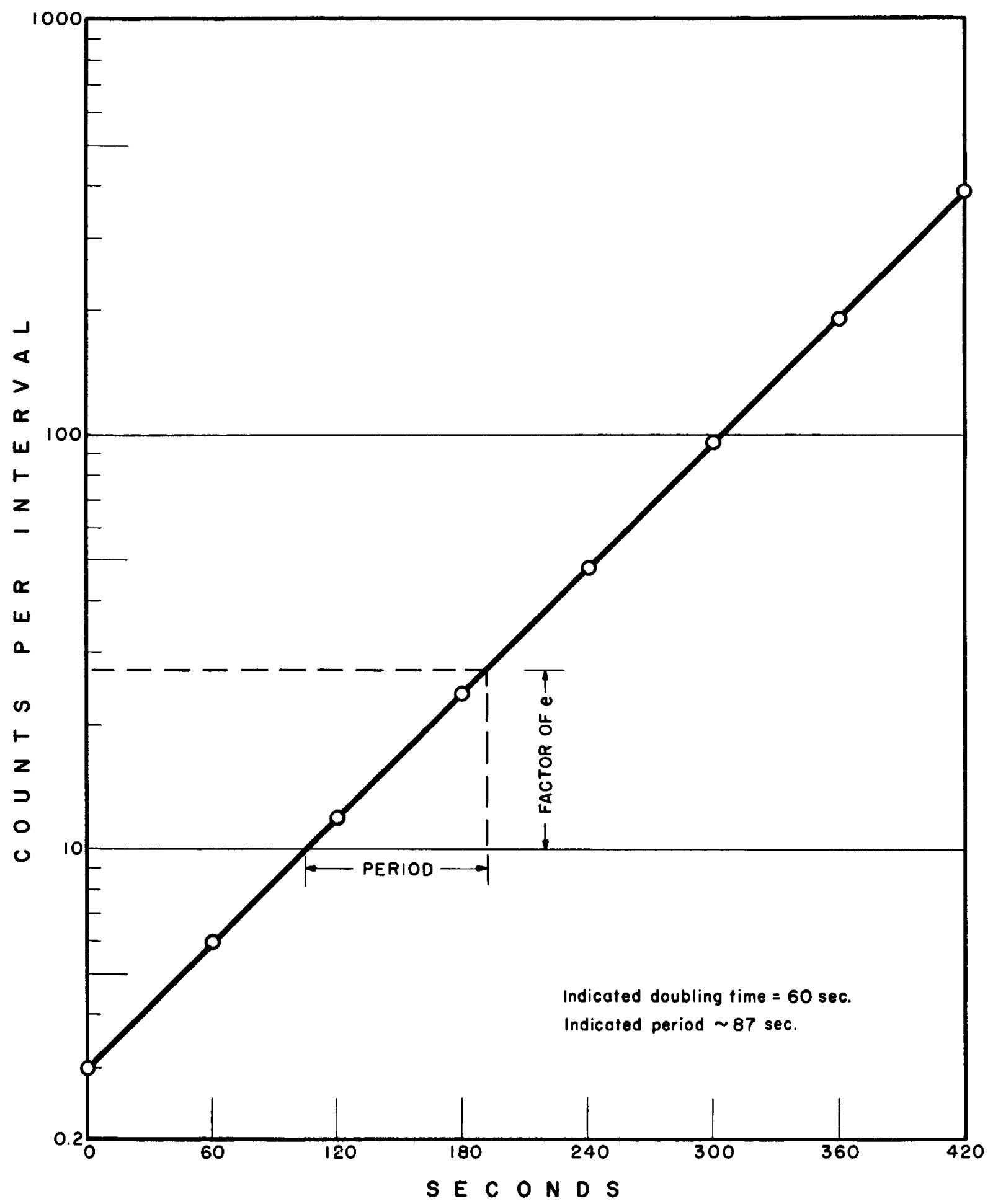

FIGURE 31

Representative Period Plot 


\section{APPENDIX I}

\section{ATOMIC WEIGHTS}

The weight listed for naturally occurring elements is the chemical atomic weight of the isotopic mixture. The weight for transuranic elements is a representative value of the isotopes which have been formed.

\begin{tabular}{|c|c|c|c|}
\hline ATOMIC NUMBER & ATOMIC WEIGHT & ELEMENT & SYMBOL \\
\hline 1 & 1.0080 & Hydrogen & $\mathrm{H}$ \\
\hline 2 & 4.003 & Helium & $\mathrm{He}$ \\
\hline 3 & 6.940 & Lithium & $\mathrm{Li}$ \\
\hline 4 & 9.013 & Beryllium & $\mathrm{Be}$ \\
\hline 5 & 10.82 & Boron & $B$ \\
\hline 6 & 12.011 & Carbon & C \\
\hline 7 & 14.008 & Nitrogen & $\mathrm{N}$ \\
\hline 8 & 16.000 & Oxygen & $\mathrm{O}$ \\
\hline 9 & 19.000 & Flourine & F \\
\hline 10 & 20.183 & Neon & $\mathrm{Ne}$ \\
\hline 11 & 23.991 & Sodium & $\mathrm{Na}$ \\
\hline 12 & 24. 32 & Magnesium & $\mathrm{Mg}$ \\
\hline 13 & 26.98 & Aluminum & $\mathrm{Al}$ \\
\hline 14 & 28.09 & Silicon & $\mathrm{Si}$ \\
\hline 15 & 30.975 & Phosphorus & $\mathbf{P}$ \\
\hline 16 & 32.066 & Sulfur & $S$ \\
\hline 17 & 35.457 & Chlorine & $\mathrm{Cl}$ \\
\hline 18 & 39.944 & Argon & A \\
\hline 19 & 39.100 & Potassium & $\mathrm{K}$ \\
\hline 20 & 40.08 & Calcium & $\mathrm{Ca}$ \\
\hline 21 & 44.96 & Scandium & Sc \\
\hline 22 & 47.90 & Titanium & $\mathrm{Ti}$ \\
\hline
\end{tabular}




\section{ATOMIC NUMBER}

23

24

25

26

27

28

29

30

31

32

33

34

35

36

37

38

39

40

41

42

43

44

45

46

47

48

49

50

51

52
ATOMIC WEIGHT

50.95

52.01

54.94

55.85

58.94

58.71

63.54

65. 38

69. 72

72.60

74.91

78. 96

79. 916

83.80

85. 48

87.63

88. 92

91. 22

92.91

95. 95

99.0

101. 1

102. 91

106. 4

107.880

112.41

114.82

118. 70

121. 76

127. 61
ELEMENT

Vanadium

Chromium

Manganese

Iron

Cobalt

Nickel

Copper

Zinc

Gallium

Germanium

Arsenic

Selenium

Bromine

Krypton

Rubidium

Strontium

Yttrium

Zirconium

Niobium

Molybdenum

Technetium

Ruthenium

Rhodium

Palladium

Silver

Cadmium

Indium

Tin

Antimony

Tellurium
SYMBOL

$\mathrm{V}$

$\mathrm{Cr}$

$\mathrm{Mn}$

$\mathrm{Fe}$

Co

$\mathrm{Ni}$

$\mathrm{Cu}$

$\mathrm{Zn}$

Ga

Ge

As

$\mathrm{Se}$

$\mathrm{Br}$

$\mathrm{Kr}$

$\mathrm{Rb}$

Sr

$\mathrm{Y}$

$\mathrm{Zr}$

$\mathrm{Nb}$

Mo

Tc

$\mathrm{Ru}$

$\mathrm{Rh}$

Pd

Ag

$\mathrm{Cd}$

In

Sn

$\mathrm{Sb}$

$\mathrm{Te}$ 


\begin{tabular}{|c|c|c|c|}
\hline ATOMIC NUMBER & ATOMIC WEIGHT & ELEMENT & SYMBOL \\
\hline 53 & 126.91 & Iodine & I \\
\hline 54 & 131. 30 & Xenon & $\mathrm{Xe}$ \\
\hline 55 & 132.91 & Cesium & $\mathrm{Cs}$ \\
\hline 56 & 137.36 & Barium & $\mathrm{Ba}$ \\
\hline 57 & 138.92 & Lanthanum & La \\
\hline 58 & 140.13 & Cerium & $\mathrm{Ce}$ \\
\hline 59 & 140.92 & Praseodymium & $\operatorname{Pr}$ \\
\hline 60 & 144.27 & Neodymium & $\mathrm{Nd}$ \\
\hline 61 & - & Promethium & $\mathrm{Pm}$ \\
\hline 62 & 150.35 & Samarium & $\mathrm{Sm}$ \\
\hline 63 & 152.0 & Europium & $\mathrm{Eu}$ \\
\hline 64 & 157.26 & Gadolinium & Gd \\
\hline 65 & 158.93 & Terbium & $\mathrm{Tb}$ \\
\hline 66 & 162.51 & Dysprosium & Dy \\
\hline 67 & 164.94 & Holmium & Ho \\
\hline 68 & 167.27 & Erbium & $\operatorname{Er}$ \\
\hline 69 & 168.94 & Thulium & $\operatorname{Tm}$ \\
\hline 70 & 173.04 & Ytterbium & $\mathrm{Yb}$ \\
\hline 71 & 174.99 & Lutetium & $\mathrm{Lu}$ \\
\hline 72 & 178.50 & Hafnium & $\mathrm{Hf}$ \\
\hline 73 & 180.95 & Tantalum & $\mathrm{Ta}$ \\
\hline 74 & 183.86 & Wolfram & W \\
\hline 75 & 186.22 & Rhenium & $\operatorname{Re}$ \\
\hline 76 & 190.2 & Osmium & Os \\
\hline 77 & 193.1 & Iridium & Ir \\
\hline 78 & 195.09 & Platinum & $\mathrm{Pt}$ \\
\hline 79 & 197.0 & Gold & $\mathrm{Au}$ \\
\hline 80 & 200.61 & Mercury & $\mathrm{Hg}$ \\
\hline 81 & 204. 39 & Thallium & $\mathrm{T} 1$ \\
\hline 82 & 207.21 & Lead & $\mathrm{Pb}$ \\
\hline 83 & 209.0 & Bismuth & $\mathrm{Bi}$ \\
\hline \multicolumn{4}{|c|}{ UNCLASSIFIED } \\
\hline
\end{tabular}




\begin{tabular}{|c|c|c|c|}
\hline ATOMIC NUMBER & ATOMIC WEIGHT & ELEMENT & SYMBOL \\
\hline 84 & 210.0 & Polonium & $\mathrm{Po}$ \\
\hline 85 & 211.0 & Astatine & At \\
\hline 86 & 222.0 & Radon & $\mathrm{Rn}$ \\
\hline 87 & 223.0 & Francium & $\mathrm{Fr}$ \\
\hline 88 & 226.05 & Radium & Ra \\
\hline 89 & 227.0 & Actinium & Ac \\
\hline 90 & 232.05 & Thorium & Th \\
\hline 91 & 231.0 & Protactinium & $\mathrm{Pa}$ \\
\hline 92 & 238.07 & Uranium & $U$ \\
\hline 93 & 237.0 & Neptunium & $\mathbf{N p}$ \\
\hline 94 & 239.0 & Plutonium & $\mathrm{Pu}$ \\
\hline 95 & 242 & Americium & Am \\
\hline 96 & 244 & Curium & $\mathrm{Cm}$ \\
\hline 97 & 247 & Berkelium & $\mathrm{Bk}$ \\
\hline 98 & 250 & Californium & $\mathrm{Cf}$ \\
\hline 99 & 253 & Einsteinium & $E$ \\
\hline 100 & 255 & Fermium & Fm \\
\hline 101 & 256 & Mendelevium & $\mathrm{Mv}$ \\
\hline 102 & 253 & Nobelium & - \\
\hline
\end{tabular}




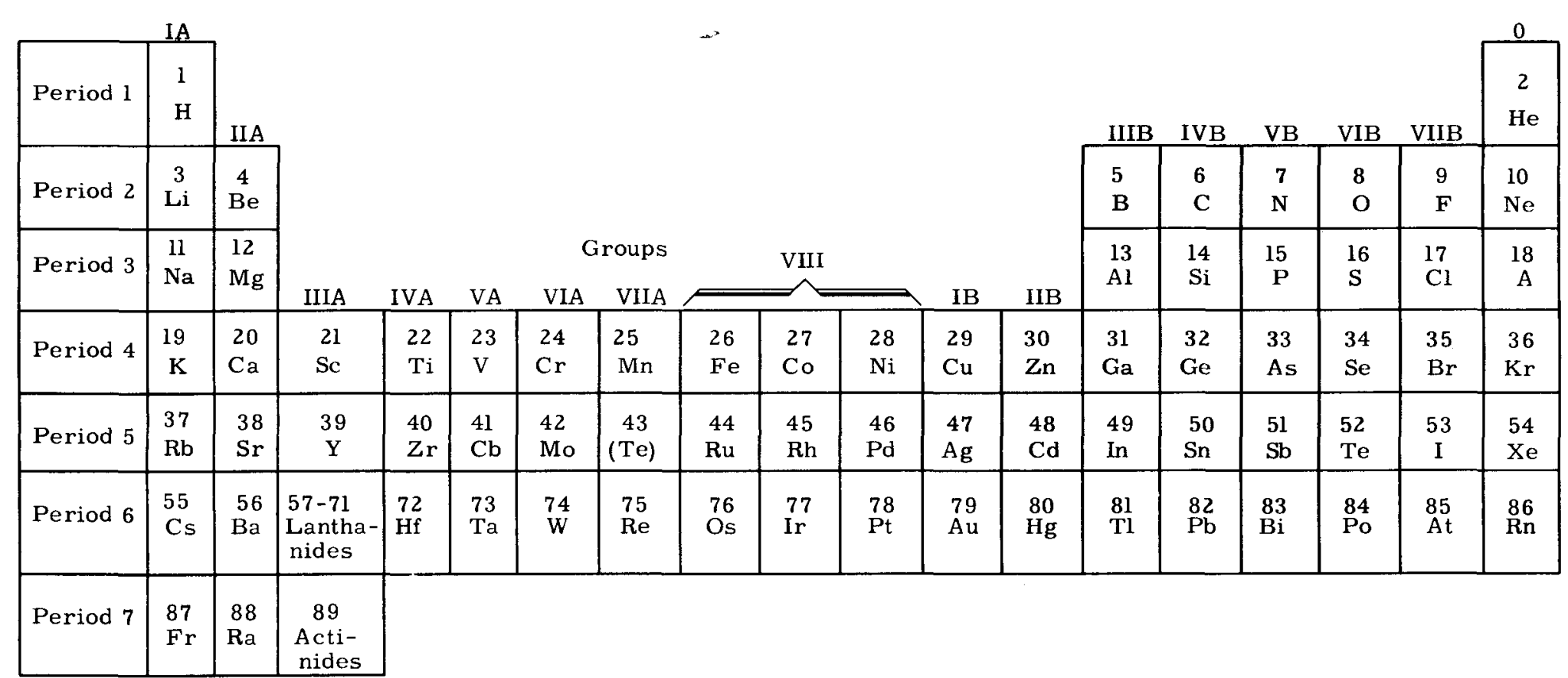

\begin{tabular}{|c|c|c|c|c|c|c|c|c|c|c|c|c|c|c|c|}
\hline $\begin{array}{c}\text { Lanthanide } \\
\text { Series }\end{array}$ & 57 & 58 & 59 & 60 & 61 & 62 & 63 & 64 & 65 & 66 & 67 & 68 & 69 & 70 & 71 \\
\hline
\end{tabular}

\begin{tabular}{|r|c|c|c|c|c|c|c|c|c|c|c|c|c|c|c|}
\hline $\begin{array}{r}\text { Actinide } \\
\text { Series }\end{array}$ & $\begin{array}{c}89 \\
\mathrm{Ac}\end{array}$ & $\begin{array}{c}90 \\
\mathrm{Th}\end{array}$ & $\begin{array}{c}91 \\
\mathrm{~Pa}\end{array}$ & $\begin{array}{c}92 \\
\mathrm{U}\end{array}$ & $\begin{array}{c}93 \\
(\mathrm{~Np})\end{array}$ & $\begin{array}{c}94 \\
(\mathrm{Pu})\end{array}$ & $\begin{array}{c}95 \\
(\mathrm{Am})\end{array}$ & $\begin{array}{c}96 \\
(\mathrm{Cm})\end{array}$ & $\begin{array}{c}97 \\
(\mathrm{Bk})\end{array}$ & $\begin{array}{c}98 \\
(\mathrm{Cf})\end{array}$ & $\begin{array}{c}99 \\
\mathrm{E}\end{array}$ & $\begin{array}{c}100 \\
\mathbf{M v}\end{array}$ & $\begin{array}{c}\mathbf{1 0 1} \\
\mathrm{Fm}\end{array}$ & $\begin{array}{c}102 \\
\mathrm{No}\end{array}$ & \\
\hline
\end{tabular}

A PPENDIX II 

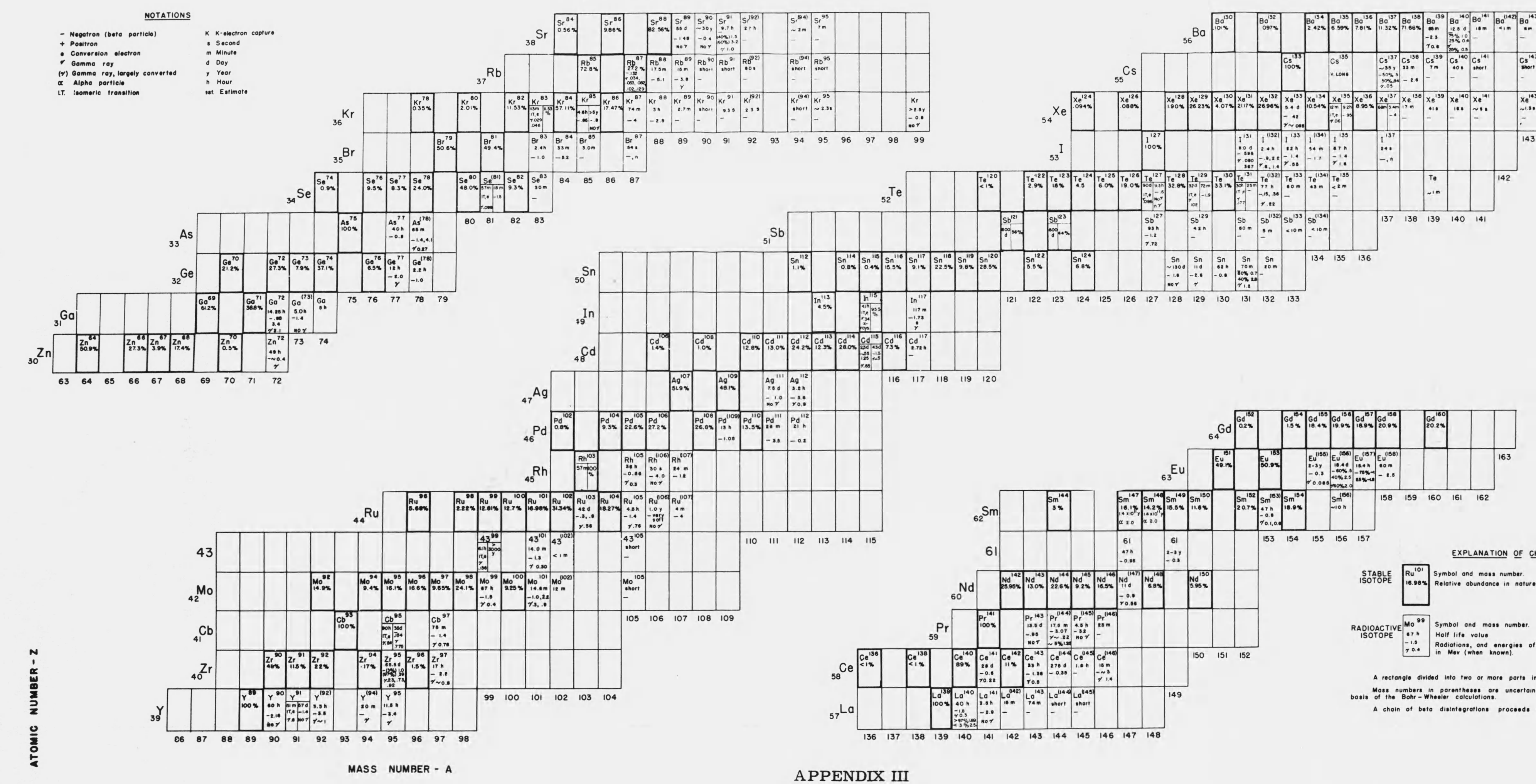

MASS NUMBER - A 


\section{DISTRIBUTION}

Copy Number

$\begin{array}{rl}1-353 & \text { Extra } \\ 354 & 300 \text { Files } \\ 355 & \text { Record Center }\end{array}$

